\title{
Antiepileptic drugs' tolerability and safety - a systematic review and meta-analysis of adverse effects in dogs
}

\author{
Marios Charalambous ${ }^{*}$, Sara K. Shivapour ${ }^{2}$, David C. Brodbelt ${ }^{3}$ and Holger A. Volk ${ }^{4}$
}

\begin{abstract}
Background: The safety profile of anti-epileptic drugs (AEDs) is an important consideration for the regulatory bodies, owners and prescribing clinicians. Information on their adverse effects still remains limited. A systematic review including a meta-analytic approach was designed to evaluate existing evidence for the safety profile of AEDs in canine patients. Electronic searches of PubMed, CAB Direct and Google scholar were carried out without date or language restrictions. Conference proceedings were also searched. Peer-reviewed full-length studies reporting adverse effects of AEDs in epileptic and healthy non-epileptic dogs were included. Studies were allocated to three groups based on their design. Individual studies were evaluated based on the quality of evidence (study design, study group sizes, subject enrolment quality and overall risk of bias) and the outcome measures reported (proportion of specific adverse effects for each AED, prevalence and $95 \%$ confidence interval of the affected population in each study and comparative odds ratio of adverse effects for AEDs).

Results: Ninety studies, including six conference proceedings, reporting clinical outcomes of AEDs' adverse effects were identified. Few studies were designed as blinded randomised controlled clinical trials. Many studies included low canine populations with unclear criteria of subject enrolment and short treatment periods. Direct comparisons suggested that imepitoin and levetiracetam might have a better safety profile than phenobarbital, whilst the latter might have a better safety profile than potassium bromide. However, none of these comparisons showed a statistically significant difference. Comparisons between other AEDs were not possible as a considerable amount of studies lacked power calculations or adequate data to allow further statistical analysis. Individual AED assessments indicated that levetiracetam might be one of the safest AEDs, followed by imepitoin and then phenobarbital and potassium bromide; these findings were all supported by a strong level of evidence. The safety profile in other AEDs was variable, but weak evidence was found to permit firm conclusions or to compare their safety to other AEDs.

Conclusions: This systematic review provides objective evaluation of the most commonly used AEDs' adverse effects. Adverse effects usually appeared mild in all AEDs and subsided once doses and/or serum levels were monitored or after the AED was withdrawn. Although phenobarbital might be less safe than imepitoin and levetiracetam, there was insufficient evidence to classify it as an AED with a high risk of major adverse effects. It is important for clinicians to evaluate both AEDs' effectiveness and safety on an individual basis before the selection of the appropriate monotherapy or adjunctive AED therapy.
\end{abstract}

Keywords: Systematic review, Meta-analysis, Epilepsy, Canine, Antiepileptic drugs, Safety, Side effects

\footnotetext{
* Correspondence: marios.charalambous.15@ucl.ac.uk

${ }^{1}$ Faculty of Brain Sciences, UCL Institute of Neurology, University College

London, London WC1E 6BT, UK

Full list of author information is available at the end of the article
} 


\section{Background}

In human medicine, a plethora of new antiepileptic drugs (AEDs) have been developed over the years for use either as monotherapy or adjunctive therapy [1]. Many of these drugs are now also used in veterinary medicine. This has led to an increase in the arsenal of AEDs used to treat canine epilepsy. As a rule, AEDs are evaluated on the grounds of their effectiveness and safety through clinical trials and experimental laboratory studies before they are approved for use in patients by the regulatory authorities, e.g. the European Medicines Agency (EMA) or the US Food and Drug Administration (FDA) [2]. The safety profile of drugs is an important consideration for their approval by the authorities and use by prescribing clinicians on their clients' animals [2, 3]. It affects clinicians' decisions to prescribe specific $\operatorname{AED}(\mathrm{s})$, as serious adverse effects can lead to chronic complications or even death. Less serious, but nonetheless important, adverse effects can significantly impact quality of life, leading to systematic illness which may increase the overall cost of treatment [3, 4]. Ultimately, the benefits of an effective AED may be outweighed by its adverse effects, and the latter should be always taken into consideration.

Many potential adverse effects for AEDs have been reported, but the evidence behind the severity of these effects or the likelihood of their occurrence has not been systematically compiled [5, 6]. Randomised clinical trials (RCTs) are a considerable source of evidence for some common or expected adverse effects [4]. However, information about serious, rare, and/or long-term adverse effects can typically be found in studies such as case reports, case series and observational studies [7, 8]. Consequently, the clinician will need to search for information from sources other than RCTs [7, 8]. Identification of all relevant studies can be time-consuming and for a busy practitioner it may be more effective to review this information via a systematic review. Systematic reviews are one of the most powerful and reliable tools to assess the severity and the probability of occurrence of AEDs' adverse effects across the spectrum of primary literature [9-12].

Although evidence for AEDs' efficacy has been recently reported and evaluated in a systematic review [13], it has been suggested that, apart from the efficacy, the selection of the appropriate AED should be also largely influenced by its safety profile [14]. To our knowledge there is only one systematic review in the field of canine epilepsy which evaluated the safety profile of a single AED, potassium bromide, across species and aetiology of seizures [15]. However, a systematic review of the adverse effects observed during treatment with any $\operatorname{AED}(\mathrm{s})$ in dogs, as well as AEDs' safety profile comparisons, has not been reported. The aim of this systematic review was to perform an objective analysis of AEDs' adverse effects in dogs, in order to provide evidence-based information on AEDs' safety profiles.

\section{Methods \\ Search strategy}

The literature search aimed to identify all studies assessing or reporting the adverse effects of an AED in dogs. Specifically, studies were evaluated based on the inclusion criteria below:

- Criterion 1-Type of study: Peer-reviewed studies in English (or translated). Experimental laboratory animal studies, clinical trials, observational and descriptive studies were included.

- Criterion 2-Case definition: For the clinical studies, dogs with IE were included as previously defined [13]. Briefly this required dogs within a certain age range, unremarkable interictal neurological status and diagnostic investigation for seizures. For the experimental laboratory animal studies (ELAS), healthy non-epileptic dogs were also included; for the latter a clear diagnostic investigation or health statement should have been reported in the study to exclude the possibility of underlying diseases.

- Criterion 3-Treatment: Dogs treated with any AED available used in canine IE were included. Doses and serum concentrations of AEDs, frequency of drug administration and treatment period were considered important information to record. Dogs treated with methods other than pharmacological intervention, e.g. homoeopathy methods, surgery, food trials, nerve stimulation, were excluded.

- Criterion 4-Outcome: Studies had to assess or report adverse effects following administration of $\operatorname{AED}(\mathrm{s})$ in canine subjects. Studies were conducted either to specifically assess or report AED(s)' safety (primary evidence studies) or to assess an outcome other than $\operatorname{AED}(\mathrm{s})$ ' safety (i.e. efficacy), while also reporting adverse effects (supportive evidence studies). Assessment of the adverse effects should have been performed by the investigators or owner.

Search strategies included use of electronic search engines for publication databases, searching of reference lists of published papers and proceedings of relevant scientific conferences. Electronic databases used were Pub Med (www.ncbi.nlm.nih.gov/PubMed), CAB Abstracts (www.cabdirect.org) and Google Scholar (www.scholar.google.com). Final electronic searches were carried out on 30 February 2015 by the primary and the second author independently, with no date or language restrictions. The search terms used in both search engines were as follows: (dog OR dogs OR canine) AND 
[(phenobarbital OR phenobarbitone OR primidone OR $\mathrm{PBr}$ OR KBr OR potassium bromide OR bromide OR nimodipine OR zonisamide OR ELB138 OR imepitoin OR levetiracetam OR verapamil OR gabapentin OR gaba OR topiramate OR felbamate OR pregabalin) OR [(treatment OR management) AND (epilepsy OR seizures)] OR (anti-convulsant OR anti-seizuring OR anti-epileptic OR AED) AND (safety OR safe OR adverse-effect OR adverse-effect OR effect OR undesirable effect OR tolerability OR toxicity OR drug toxicity OR reactions OR disease). Hand searching for articles from the reference lists of publications and searching major veterinary neurology conference meeting proceedings from 1970 to 2015 and relative textbook chapters was carried out by the primary and second authors independently. Conference proceedings were searched for the Annual Congresses of the European Society and College of Veterinary Neurology (ESVN/ECVN) and the American College of Veterinary Internal Medicine (ACVIM). Other conference proceedings were searched only if the reference list of identified publications indicated this. All items returned by the search engines, hand searches and correspondence were recorded and entered into the screening process.

\section{Study selection}

Restrictions based on publication date or language were not imposed. Studies written in non-English language were assessed initially based on an English translation (Google Translate software) and then verified by a veterinarian fluent in the language of publication.

A two-stage screening process was used [13] and the process was performed by the primary author. Firstly, studies of relevance to the systematic review objectives were identified (stage 1) and, secondly, studies likely to provide evidence of the highest available quality and sufficient detail for assessing the outcome measures and methodology were selected (stage 2). Stage 1 of the screening process identified from the total search results any studies that: (a) fulfilled inclusion criterion 1 and (b) reported findings related to the adverse effects and safety of AEDs administered in dogs. Stage 1 assessment evaluated the retrieved papers' titles and abstracts only. At stage 2, papers were selected for full data extraction according to the inclusion criteria 2,3 and 4 and were evaluated in detail on the grounds of the quality of evidence and outcomes by MC.

\section{Assessment of quality of evidence}

Blinded RCTs (bRCTs) and blinded randomised ELAS (bRELAS) were considered most likely to produce higher quality evidence, followed by non-blinded RCTs (nbRCTs) and non-blinded randomised ELAS (nbRELAS), then non-randomised clinical trials (NRCTs) and non-randomised ELAS (NRELAS), uncontrolled clinical trials (UCTs) and uncontrolled ELAS (UELAS), cohort, case-control and cross sectional studies and lastly case series and reports [16-18]. Accordingly, the studies were allocated based on their design to one of three groups, i.e. bRCTs, bRELAS, nbRCTs and nbRELAS (first group), NRCTs, NRELAS, UCTs, UELAS, cohort, case-control and cross-sectional studies (second group) and case series and reports (third group).

As a general rule, the studies in the first group (bRCTs and bELAS in particular) were considered to provide higher quality evidence, followed by the studies in the second and third group. In addition, a three-part system of evidence quality assessment to indicate the strengths and weaknesses of each study within each group was used [13, 19]: (a) study group sizes, (b) subject enrolment quality and (c) overall risk of bias based on Cochrane [20] and Syrcle's [21] 'risk of bias' assessment tool in order to provide an indicator of confidence associated with the findings of each study. For instance, bRCTs or bRELAS with large group sizes, clear inclusion criteria, thorough diagnostic investigations and low overall risk of bias were considered to provide the highest available quality of evidence.

\section{Study group sizes}

This characteristic was categorized for each study using the following system $[13,19]$ : (a) $>50$ subjects per group ('good' number of subjects), (b) 20-50 subjects per group ('moderate' number), (c) 10-19 subjects per group ('small' number) and (d) $<10$ subjects per group ('very small' number).

\section{Assessment of subject enrolment quality}

Data on investigations to reach the diagnosis of IE were retrieved to evaluate the quality of subject enrolment in each study as 'well characterized', 'fairly characterized', 'poorly characterized' or 'unclear.' Well characterized diagnoses were defined as diagnostic investigations that included clinical signs and thorough test results consistent with the diagnosis of IE; specifically, the signalment, the absence of neurological deficits between the ictal phases, unremarkable routine biochemical and haematological blood tests and imaging results (including brain MRI and/or CT) and/or normal cerebrospinal fluid (CSF) analysis for all cases of the study. Fairly characterized, used for intermediate situations, were defined as diagnostic investigations that were based on signalment, clinical examination and basic diagnostic investigation (i.e. blood tests only), with only some study cases having had advanced brain imaging and/or CSF analysis. Poorly characterized were defined as diagnostic investigations that were based on signalment, clinical examination and/or basic diagnostic investigation (i.e. blood tests) 
only. Unclear related to reports where the approach to diagnosis of IE was not clearly stated (e.g. when clinical signs were not stated and insufficient or no details of diagnostic tests were provided or when dogs with IE were included without reporting details on diagnostic investigation).

For the ELAS, which included non-epileptic healthy animals, 'clearly characterized' were the studies that defined diagnostic investigations and thorough test results to exclude any systemic illness; 'unclear' were characterized when diagnostic investigations to rule out diseases were not clearly stated or when dogs were included and considered healthy without reporting details on diagnostic procedures.

\section{Assessment of overall risk of bias}

The overall risk of bias in the clinical trials was assessed based on the criteria of the Cochrane 'risk of bias' assessment tool [20]. Syrcle's 'risk of bias' assessment tool [21] was used to assess the overall risk of bias in ELAS. The latter tool is an adapted version of the Cochrane one and was designed to facilitate critical appraisal of evidence from ELAS.

Each of the following study components was categorized as presenting a 'high', 'low' or 'unclear' risk of introducing bias to the study findings: random sequence generation, allocation concealment, blinding of participants and personnel, blinding of outcome assessment, completeness of outcome data, selective reporting of outcomes and other sources of bias. For ELAS, two further components-random housing and baseline characteristics of dogs - were also assessed and mentioned as part of the "other sources of bias" section. Case series and reports as well as observational studies were considered to be of high overall risk of bias.

\section{Level of the studies' evidence}

The level of evidence provided for the safety profile of each AED was based on the overall quality of evidence of the studies. The level of evidence was allocated according to a previous similar system $[13,19]$ which was extensively modified for the needs of the current study: 'strong' evidence was provided for the safety profile when at least one bRCT and/or bRELAS reported or assessed the adverse effects of an AED; 'weak' evidence was provided for the safety profile when bRCTs and/or bRELAS were not available.

\section{Assessment of outcome measures}

The outcome measure of this study was the evaluation of the safety profile of $\mathrm{AED}(\mathrm{s})$ administered in dogs. The adverse effects were organized by body system (e.g. neurological, gastro-intestinal, dermatological, etc.) and types, including type I (dose dependent and predictable) and type II (idiosyncratic-dose independent and unpredictable). Different terms used by the studies but describing the same adverse effects (e.g. drowsiness and somnolence, wobbly gait and ataxia, lethargy and sedation, etc.) were considered synonymous and only one term was selected for use in the analysis. The outcome measure was assessed according to the methods below:

\section{Proportion of specific adverse effects for each AED}

This was expressed as a percentage and calculated for each AED by dividing the number of studies that reported a specific adverse effect by the total number of the studies for this AED. If an AED was used as a monotherapy and adjunctive therapy, further calculations were also performed for each sub-category.

\section{Prevalence and $95 \%$ confidence interval of the affected population in each study}

Prevalence was expressed as a percentage and calculated for each study by dividing the number of subjects that developed adverse effects during the specified study period by the total size of the study population. The $95 \%$ confidence interval $(95 \% \mathrm{CI})$ of the proportion of study animals that developed adverse effects related to the $\operatorname{AED}(\mathrm{s})$ was calculated by standard methods [22] This was used as a further indicator of an AED's safety profile. If the $95 \% \mathrm{CI}$ of affected dogs (based on $95 \%$ CI calculations) were $\geq 50 \%$, then it was considered that the majority of the study population experienced adverse effects.

For each study, the period of treatment, AED's doses and serum levels were reported with the aim to evaluate the association of these values with the prevalence of each AED's adverse effects.

\section{Statistical analysis}

For the comparison groups' studies, a further approach was conducted to identify statistical differences between studies with regards to reported adverse effects. For each AED study, the total number of patients experiencing adverse effects and/or the number of patients experiencing specific adverse effects (e.g. sedation, ataxia, polyuria, etc.) in all therapeutic groups were retrieved. The odds ratio (OR) was then estimated in order to indicate the increased or decreased odds of observing a specific adverse effect(s) in total for an AED compared to its control group (comparison AED or placebo or untreated animals). Statistical analysis was undertaken following the guidelines of the Handbook of the Cochrane Collaboration 5.0. The OR for dichotomous data was calculated using the random-effects model in Review Manager 5.3. Heterogeneity between studies was calculated using the Chi square test and was considered to be heterogeneous when $\mathrm{P} \leq 0.1$. $\mathrm{I}^{2}$ values of no more than 
25,26 to $74 \%$ and no less than $75 \%$ were considered as "low", "moderate" and "high" heterogeneity, respectively. Associations were considered to be statistically significant at $\mathrm{P}<0.05$. $\mathrm{P}$ values between 0.05 and 0.1 were considered as statistical trends of potential interest.

\section{Results}

\section{Description of studies}

By 29 December 2015, the search strategy had identified a total of 368 unique citations; 347 from the electronic searches of PubMed, CAB Abstracts, Google Scholar and manual searches from the publications' reference lists, 16 from manual searching of major conference proceedings and 6 unpublished studies included as part of published data. Two hundred ninety two items fulfilled stage 1 screening criteria. Of these, 90 final studies (published between 1981 and 2015) also fulfilled stage 2 selection criteria and were thus selected for review.

The vast majority of studies were allocated in the second (i.e. non-blinded, non-randomised and uncontrolled studies) and third (i.e. retrospective case series and reports) group. A few studies included more than one sub-study (i.e. a clinical trial and/or ELAS and/or retrospective case series part); accordingly, such studies were included in more than one group. Therefore, study designs represented were five bRCT [23-27], two nbRCT $[28,29]$ and seven nbRELAS $[25,30-35]$ in the first group, six NRCTs [36-41], 11 NRELAS [42-52], 22 UCTs [44, 48, 53-71], six UELAS [34, 72-76] and one cross sectional study [3] in second group, and 19 retrospective case series [77-95] and 16 case reports [96-111] in the third group. In addition, five unpublished studies described adverse effects and were reported briefly in EMA report; thus all these were considered as one study [112] and were not included in any category as there was insufficient information as far as their design was concerned.

Overall, the 90 selected studies reported 12 AEDs. In all studies but one [43], the AEDs were orally administered. Within each study, one or more AEDs were evaluated as a monotherapy and/or adjunct to other AEDs.

\section{Disease characterisation}

In the majority of the studies, the inclusion criteria for diagnosing IE were not well characterized. According to the described grading system for subject enrolment quality, 16 studies $[27,33,53,61,63,64,68,80,81,90,96$, $97,100,101,110,111]$ enroled treatment groups of well characterized IE, 13 studies [3, 44, 48, 54-57, 62, 66, 67, 77-79] enroled treatment groups of fairly characterized IE, and 14 studies $[23-26,39,58,59,65,74,82,84,88$, $108,109]$ enroled treatment groups of poorly characterized IE. In 26 studies [28, 29, 36-38, 50, 60, 69, 70, 75,
83, 85-87, 89, 91-94, 98, 99, 102-106], the diagnostic procedures for enrolment of cases with IE were unclear.

As far as the ELAS including healthy animals were concerned, eight [31, 36, 45, 46, 50-52, 73] enroled treatment groups of clear and 14 [25, 30, 32, 34, 35, 37, $38,42,43,47,49,72,76$ ] enroled treatment groups of unclear or unknown diagnostic investigation for ruling out other diseases. In one report, a dog was nonepileptic and was treated with phenobarbital and chlomipramine due to anxiety and aggression, but the diagnostic investigation for this was unclear [107].

\section{Study group sizes}

The vast majority of studies reported the total number of dogs evaluated. The majority of studies evaluated small or very small study size groups. Thirteen studies $[25,26,40,50,62,69-71,75,82,88,90,113]$ evaluated groups with a good number of dogs, 13 studies [23, 24, $32,37,39,65,74,77,79,80,91,94,95]$ evaluated groups with a moderate number of dogs, 26 studies [3, 28, 34, $36,38,44-46,48,53,54,56-61,63,64,66,70,75,81$, $83,84,114$ ] evaluated groups with a small number of dogs and 38 studies [33-35, 39, 42, 43, 51-53, 55, 67, $68,72,73,76,78,85,87,89,92,93,96-100,102-111$, $115,116]$ evaluated groups with a very small number of dogs. In two studies, the study group size was unclear $[47,49]$.

Signalment and baseline characteristics of study subjects Baseline characteristics (such as breed, age and sex) of total enroled dogs were reported to some extent for all 90 studies. Clear presentation of statistical comparison of intervention groups with respect to signalment and baseline disease characteristics was not commonly encountered.

In all studies reporting baseline data, the recruited dogs represented multiple breeds, both sexes and a wide range of ages at study entry (median 5, mean 4, range 0.5-7 years). Major affected breeds were crossed-breeds and pure breeds such as Labrador and Golden Retrievers followed by German Shepherd dogs, Beagles, Boxers and Poodles. In the majority of the studies more males were affected compared to females, though these differences were not evaluated statistically.

\section{Methodological quality of included studies}

The vast majority of studies revealed high and/or unclear risk of bias for all the components (Fig. 1). As stated in the methods, retrospective case series and reports were not included in the methodological quality assessment as these were considered to be at an overall high risk of bias. 


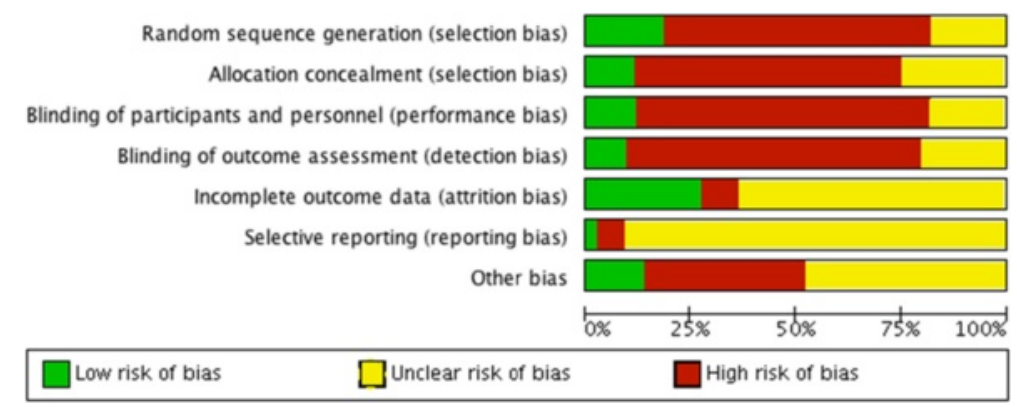

Fig. 1 Risk of bias. Risk of bias assessment presented as percentages across all included studies

\section{Randomization and allocation concealment}

Studies in group A used randomization to allocate the dogs and were considered to provide a low risk of bias. Eight studies [25, 28, 29, 32-35, 68] did not offer enough detail to confirm that allocation concealment was performed. Five studies stated that randomization was concealed without further details. Two studies [23, 27] assigned by random blocking (random allocation to blocks of 10 and 6 respectively). One study [27] used pre-defined randomization lists under double-blinded conditions. One study [31] used drawing lots and two studies $[24,30]$ used a computer-generated list of random numbers.

\section{Blinding of outcome assessment}

Only in five studies [23-27], in group A, blinding was clearly described; these were also considered to be at low risk. In these five studies, blinding was applied to all participants, personnel and outcome assessment. In one of them [23] all but the primary investigator were blinded.

\section{Incomplete outcome data}

Ten studies presented outcome data from all enroled dogs in the treatment group to which they were originally allocated and there were no losses between enrolment and evaluation $[30,33,42,51,53,59,61,67,73$, 78 . The same studies were considered to be at low risk of bias. In two studies, it was unclear whether all dogs completed the study, as inadequate information was provided $[60,64]$. Across the remaining studies, there were dogs that were euthanized or excluded due to poor seizure control, owner request or for unidentified reasons; thus there were losses between the initial study populations and the final number of the dogs.

\section{Selective reporting}

It was difficult to assess selective reporting as study protocols were not sought beyond the information published. In two reports $[29,112]$ further information was attempted to be retrieved but complete protocols were never obtained.

\section{Acknowledgment of other sources of bias}

Twelve studies reported financial support [24, 26-28, 41, $44,53,58,61,68,73,79]$ but there was not adequate evidence to support whether this biased the results. One study [54] clearly mentioned that there was no financial support, while the remaining studies failed to report financial support.

In two studies $[28,78]$ the statistical analysis was not clarified. In one study [25], many dogs were excluded from both groups mainly for treatment-related reasons (post-randomisation bias). Six studies [29, 49, 60, 64, 70, $86]$ were conference abstracts, thus no further information could be retrieved. One dog in one study [66] and two dogs in two studies [28, 63] were diagnosed with symptomatic epilepsy (i.e. a cause was identified); this could potentially affect the final results on AED safety profile. Conflict of interest was clearly stated in one study [25].

In the ELAS, specifically, nine studies [25, 30, 33, 34, $42,44,51,52,68]$ reported details for the experimental dogs' housing. Random housing of the dogs occurred in all but nine studies [34, 35, 37, 42-44, 49, 72, 73]. The baseline characteristics of the dogs were reported in nine studies $[25,30,33,42,44,51,52,68,73]$ and were similar for all the experimental groups in seven studies $[25,30,33,42,44,51,52]$.

\section{AEDs safety profile}

\section{A) Safety profile for each AED individually}

Proportions of adverse effects for each AED are summarized in the text and presented in Figs. 2, 3, 4, 5, 6, 7, 8, $9,10,11$. Details of doses and serum concentrations of $\mathrm{AED}(\mathrm{s})$, period of treatment as well as prevalence of adverse effects and $95 \% \mathrm{CI}$ of the proportion of affected cases (included type and most frequently occurred) for each study are summarized in the text and provided in detail in Tables 1, 2, 3, 4, 5, 6, 7. 


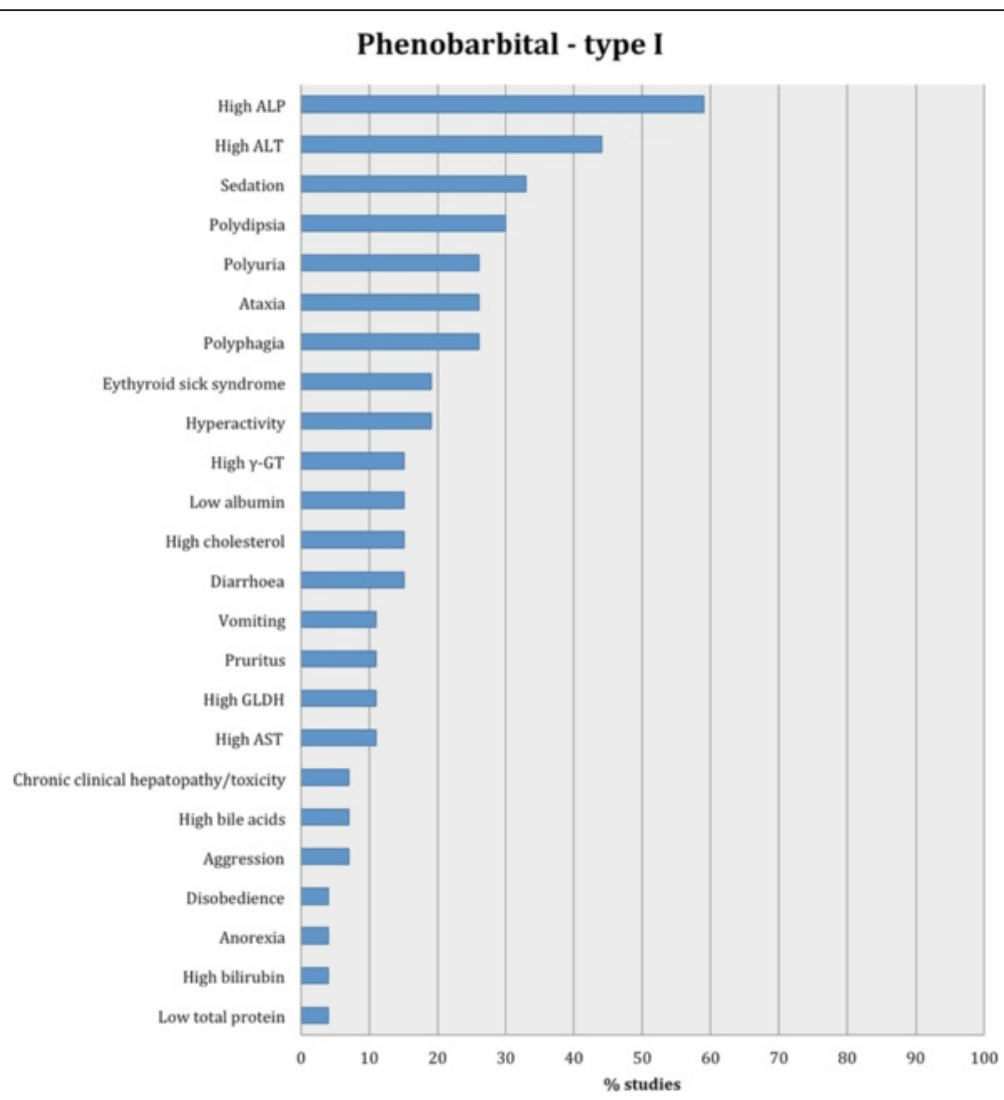

Fig. 2 Proportion of specific type I adverse effects for phenobarbital. Each adverse effect represents the percentage of studies that reported this specific adverse effect for phenobarbital monotherapy

\section{Phenobarbital}

There was an overall strong level of evidence provided for the phenobarbital safety profile. Forty-three studies $[3,23,25,27-29,31,33,36-40,42,43,45,46,49,50$, $52,53,60,69,71,74-76,79-81,83-85,87,93-95,98$, $100,103,107,109,111]$ presented data about the safety profile of phenobarbital as a monotherapy agent, giving a combined sample size of 1003 dogs.

Twenty-seven studies reported type I adverse effects (dose dependent/predictable), including neurological signs and clinical pathological findings as the most common (Table 1). Specifically, these adverse effects most

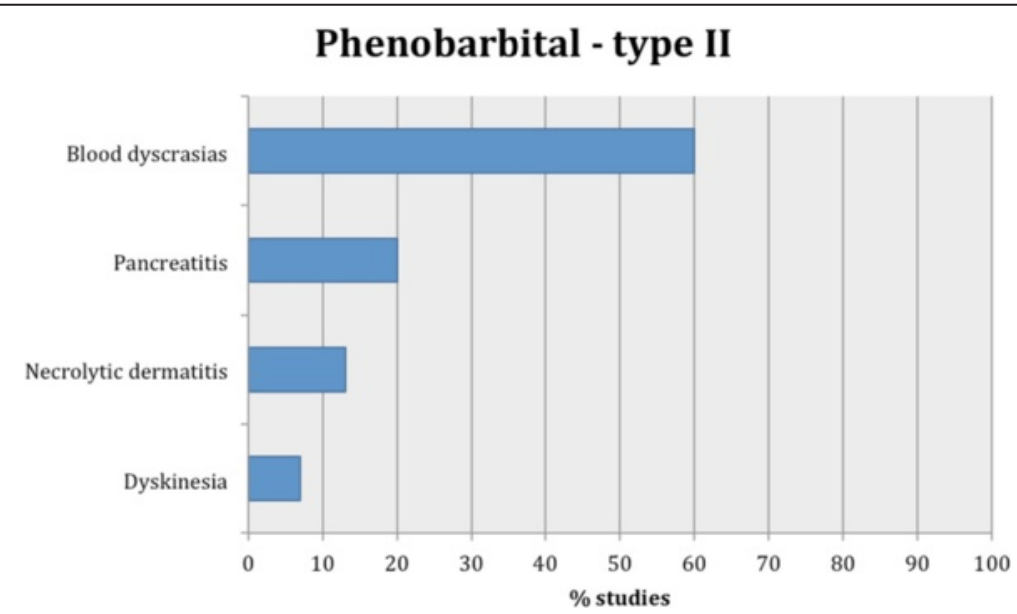

Fig. 3 Proportion of specific type II adverse effects for phenobarbital. Each adverse effect represents the percentage of studies that reported this specific adverse effect for phenobarbital monotherapy 


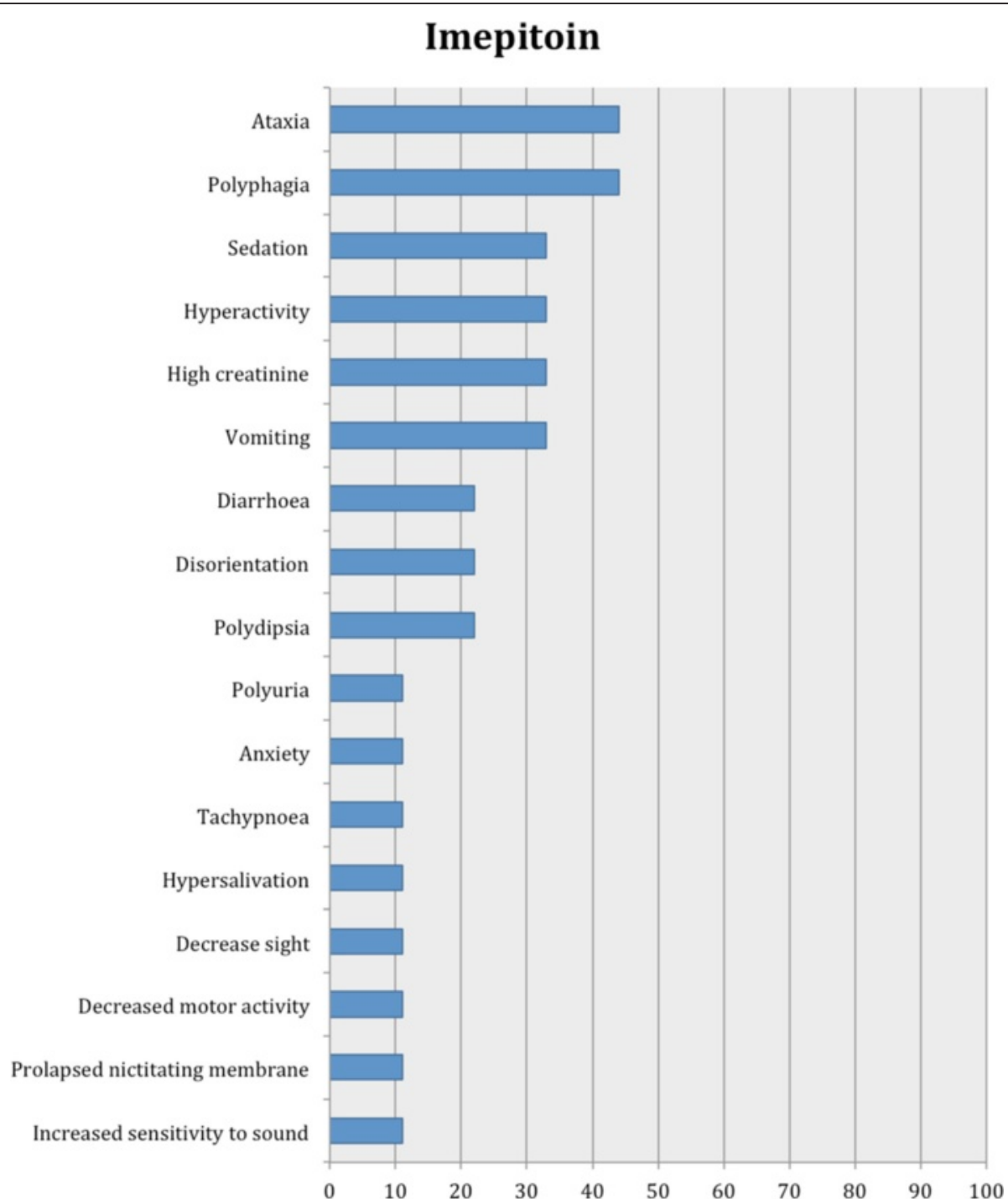

Fig. 4 Proportion of type I adverse effects for imepitoin. Each adverse effect represents the percentage of studies that reported this specific adverse effect for imepitoin monotherapy

commonly included increased serum ALP activity and ALT activity followed by sedation, ataxia, polydipsia, polyuria, polyphagia, euthyroid sick syndrome, hyperactivity, increased serum $\gamma$-GT activity, decreased serum albumin and diarrhoea. Less commonly, vomiting, pruritus, chronic clinical hepatopathy/toxicity, increased serum GLDH activity, AST activity, cholesterol, bile acids and bilirubin activity, aggression, anorexia and decreased serum total proteins were reported (Fig. 2). Twenty studies reported alterations in one or multiple liver enzymes, but only three of them reported chronic clinical hepatopathy/toxicity. The occurrence of euthyroid sick syndrome and asymptomatic/subclinical pancreatitis may have been underestimated because only a very few studies included the relevant diagnostic tests to evaluate these disorders. Two studies [31,33] evaluated the effect of phenobarbital on adrenal function and found no significant effect. The same studies reported no adverse effects, although they focused on reporting adverse effects related to adrenal function. One study [71] reported that adrenal function might have been affected by phenobarbital (i.e. altered ACTH stimulation and dexamethasone suppression tests despite normal endogenous ACTH concentrations).

Fifteen studies reported type II adverse effects (idiosyncratic/unpredictable) with hematological signs as the most common (Table 1). Specifically, the most commonly reported adverse effects included blood dyscrasias (i.e. anemia, thrombocytopenia, leucopenia and/or pancytopenia) followed by pancreatitis, superficial necrolytic dermatitis and lastly dyskinesia (i.e. twitching episodes) (Fig. 3). In one study [50] elevated serum canine 


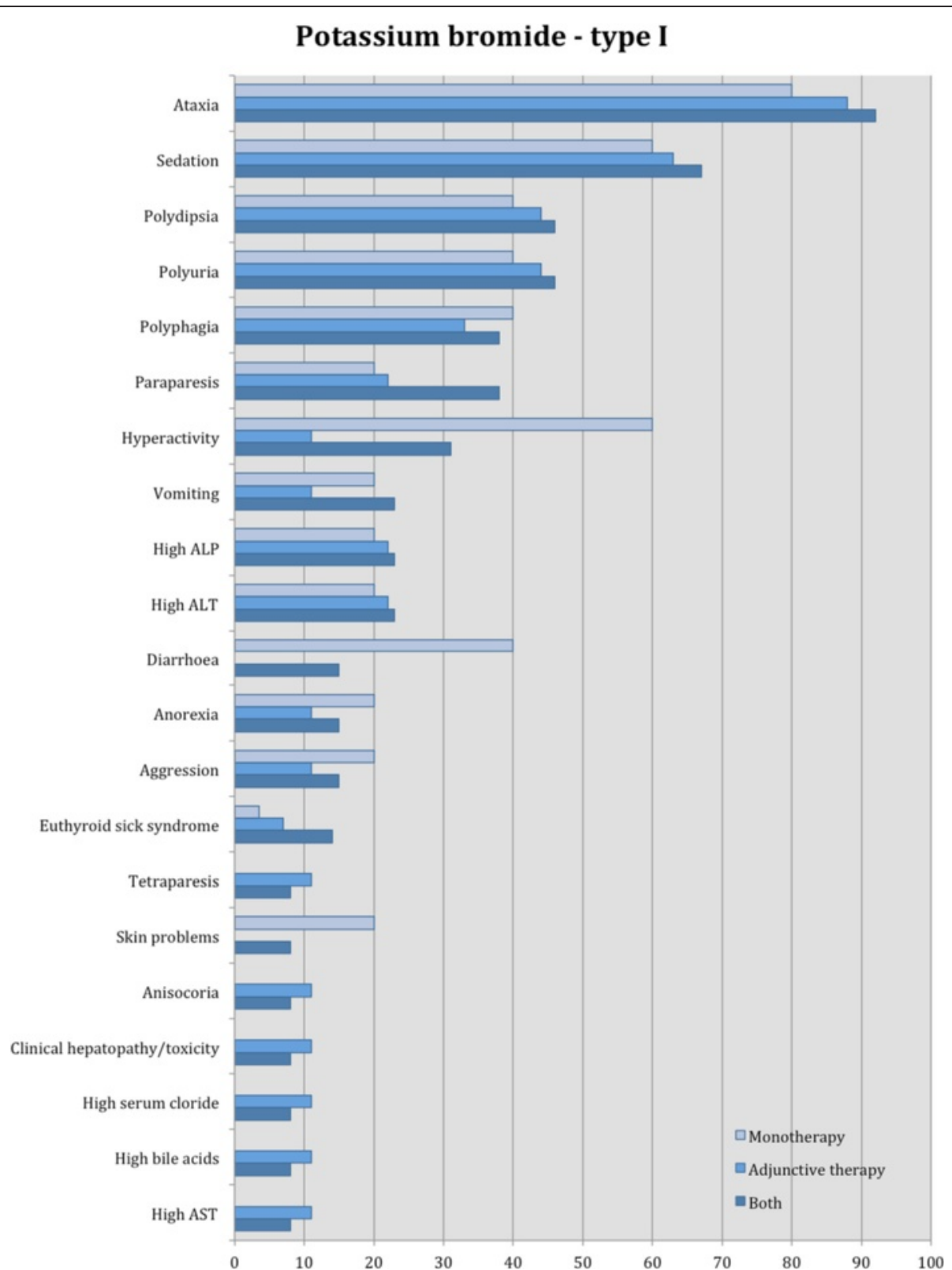

Fig. 5 Proportion of specific type I adverse effects for potassium bromide. Each adverse effect represents the percentage of studies that reported this specific adverse effect for potassium bromide monotherapy and adjunctive therapy

pancreatic lipase immunoreactivity (cPLI) concentration was detected in some dogs, but further diagnostic tests and clinical evaluation was not performed; thus, it is unknown if the dogs had developed clinical pancreatitis. In addition, in one study [81], 212 dogs with IE treated with phenobarbital monotherapy or combined therapy were evaluated and only 9 were found to have phenobarbital-induced blood dyscrasias, giving a prevalence of $4.2 \%$ (95 \% CI: 1.5-7.0 \%).

Adequate data to allow calculations of the prevalence of adverse effects was reported in 25/43 (58 \%) of the studies (Table 1). From these, 13/25 (52 \%) showed $>50 \%$ prevalence of at least one adverse effect for the specific period of treatment they were conducted. Based on the $95 \% \mathrm{CI}$, the majority of the study populations experienced at least one adverse effect in 11/25 (44\%) studies (Table 1).

Adequate information about the treatment period was reported in 34/43 (79 \%) (Table 1). From these, in 10/34 $(29 \%)$ the treatment period was relatively short ( $<6$ months). Adequate information about the dose was provided in 28/43 (65\%) studies (Table 1). From these, 19/28 (68 \%) and 9/28 (32\%) reported type I and type II adverse effects respectively. The maintenance doses were 


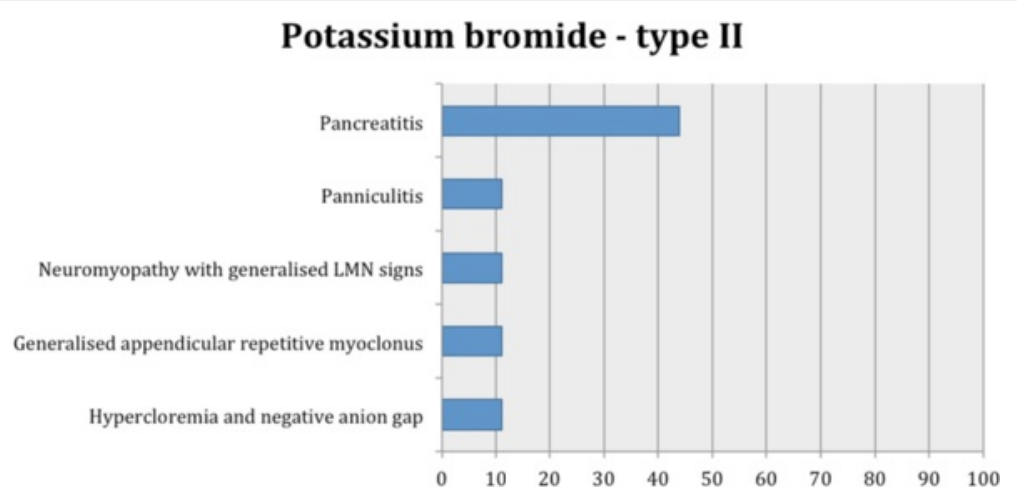

Fig. 6 Proportion of specific type II adverse effects for potassium bromide. Each adverse effect represents the percentage of studies that reported this specific adverse effect for potassium bromide monotherapy and/or adjunctive therapy

higher than the recommended dose range in type I adverse effects $(11 / 19,58 \%)$, while within normal ranges for type II adverse effects (7/9, $78 \%)$. Adequate information about the phenobarbital serum levels was reported in $24 / 43(56 \%)$ studies (Table 1). From these $17 / 24$ (71 \%) and 4/24 (17\%) reported doses for type I and type II adverse effects respectively. The phenobarbital serum levels were higher than the recommended therapeutic ranges in type I adverse effects $(9 / 17,53 \%)$, while within normal ranges for type II adverse effects (4/4, $100 \%)$.

\section{Imepitoin}

There was an overall strong level of evidence provided for the imepitoin safety profile. Six studies $[25,26,44$, $48,112,113]$ presented data about the safety profile of oral imepitoin either as monotherapy (all studies) and/or an adjunct to other AEDs (two studies) [44, 48], giving a combined sample size of 458 dogs. Two studies [25, 44] included both a clinical trial and ELAS part. EMA imepitoin assessment report [112] included two unpublished GLP toxicity studies and three unpublished clinical trials [117-119]; the latter three studies were not clearly distinguished in the EMA report and thus they were considered as one study.

Nine studies reported type I adverse effects including neurological and gastro-intestinal signs as the most common (Table 2). Specifically, the most common adverse events included ataxia and polyphagia, followed by sedation, hyperactivity, increased serum creatinine activity, vomiting and diarrhoea, disorientation and polydipsia. Less commonly, polyuria, anxiety, tachypnea,

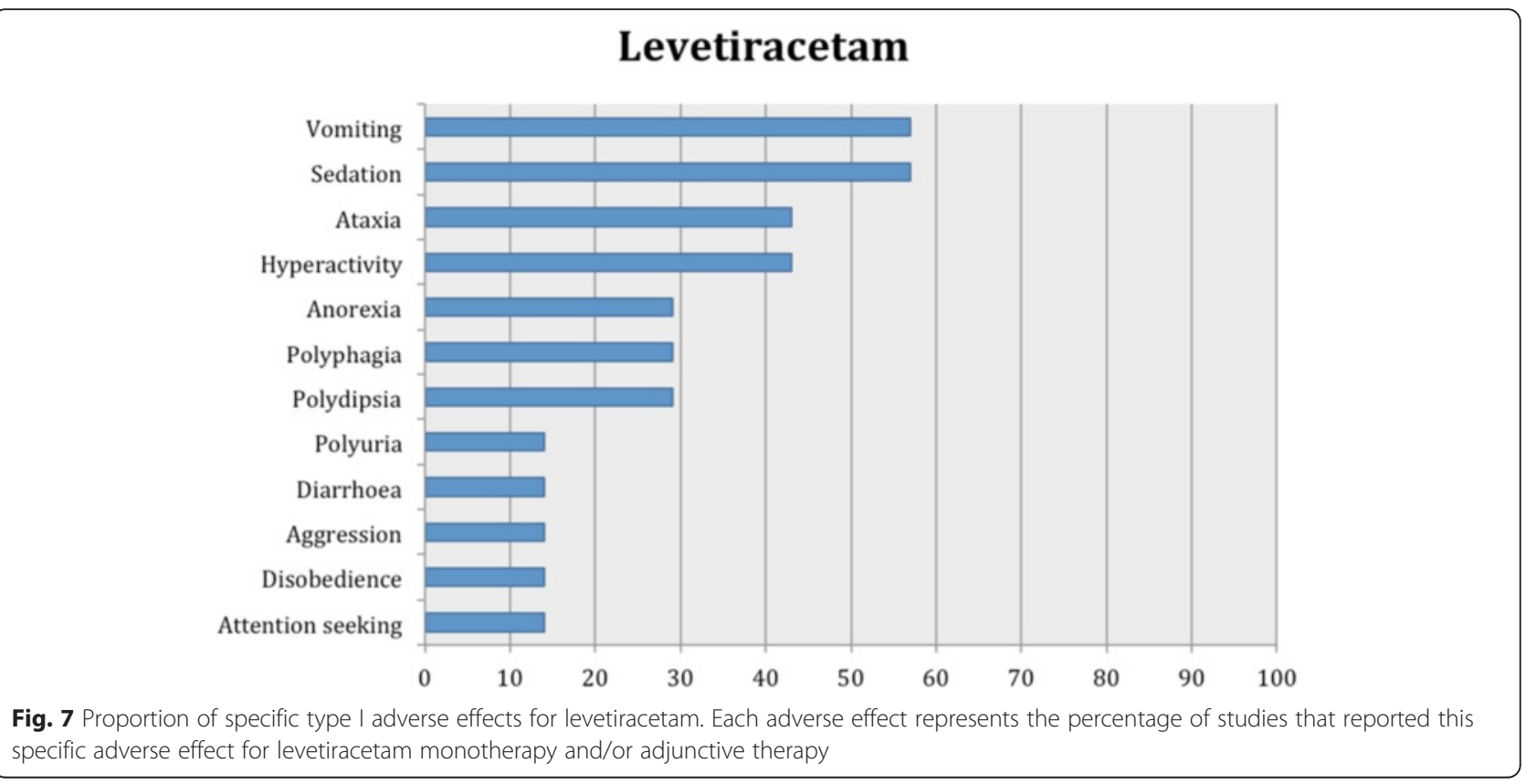




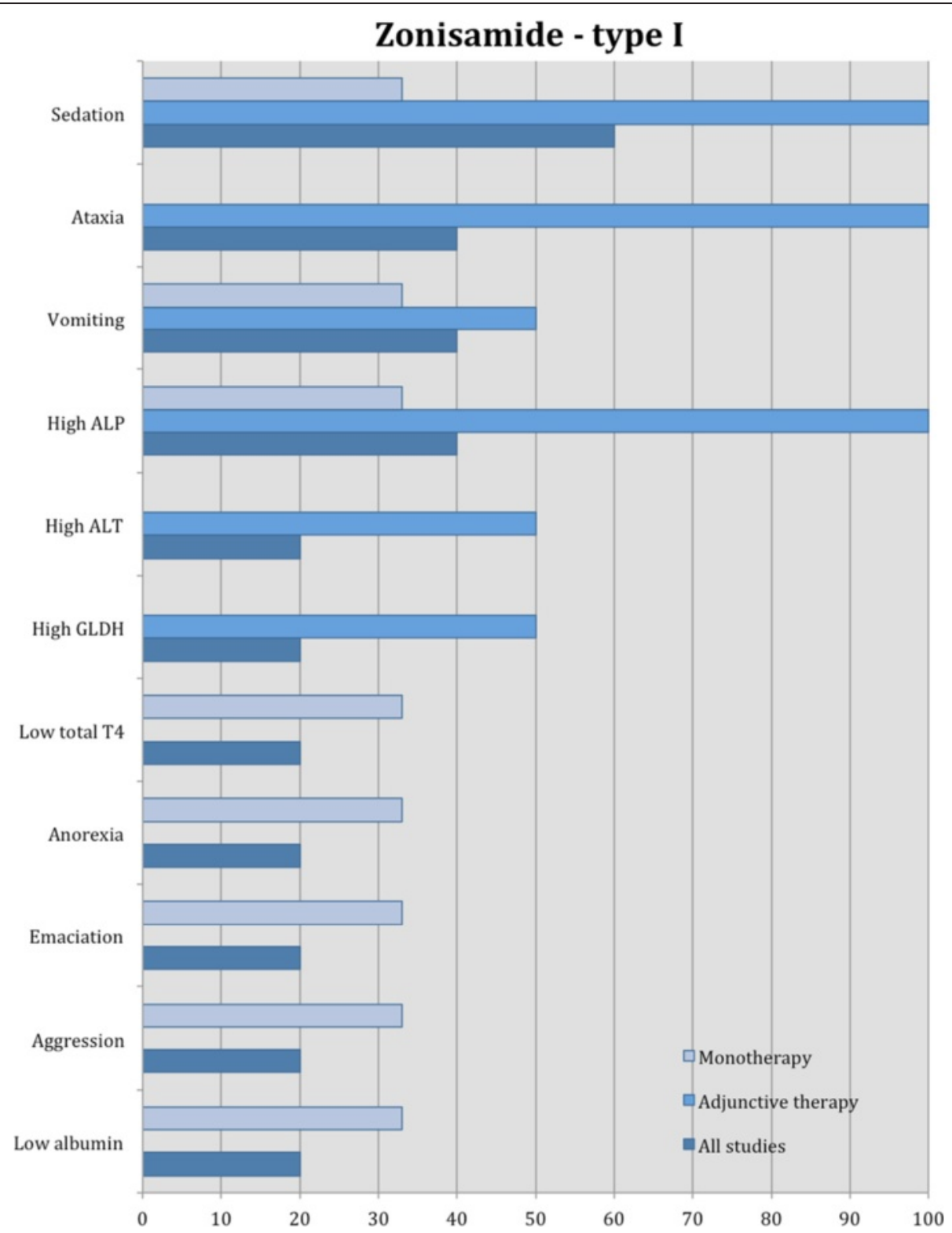

Fig. 8 Proportion of specific type I adverse effects for zonisamide. Each adverse effect represents the percentage of studies that reported this specific adverse effect for zonisamide monotherapy and adjunctive therapy

hypersalivation, decrease in sight and motor activity, prolapsed nictitating membrane and increased sensitivity to sound were reported (Fig. 4).

It is worth mentioning that the two GLP toxicity studies [112] and the ELAS part of one study [25] reported a few further adverse effects. However, these specific adverse effects were not included in the assessment above (see Table 2 for details) because higher than therapeutic doses were administered ( $3 \mathrm{X}, 5 \mathrm{X}$ or higher the recommended dose) which intended to evaluate the potential toxicity and tolerability of the drug. The same studies showed no adverse effects when imepitoin was administered in the recommended doses $(\leq 30 \mathrm{mg} / \mathrm{kg})$ apart from vomiting, hypersalivation and prolapsed nictitating membrane (as described above).

Adequate data to allow calculations of the prevalence of adverse effects was reported in 5/9 (55\%) of the studies (Table 2). From these, only two studies showed $>50 \%$ prevalence of adverse effects for the specific treatment periods within which they were conducted. However, one of the studies included a group of dogs receiving imepitoin adjunctive therapy to phenobarbital or primidone and therefore the prevalence may have been overestimated in these. Based on the $95 \%$ CIs, the majority of the combined study populations experienced adverse effects in only one study (Table 2). 


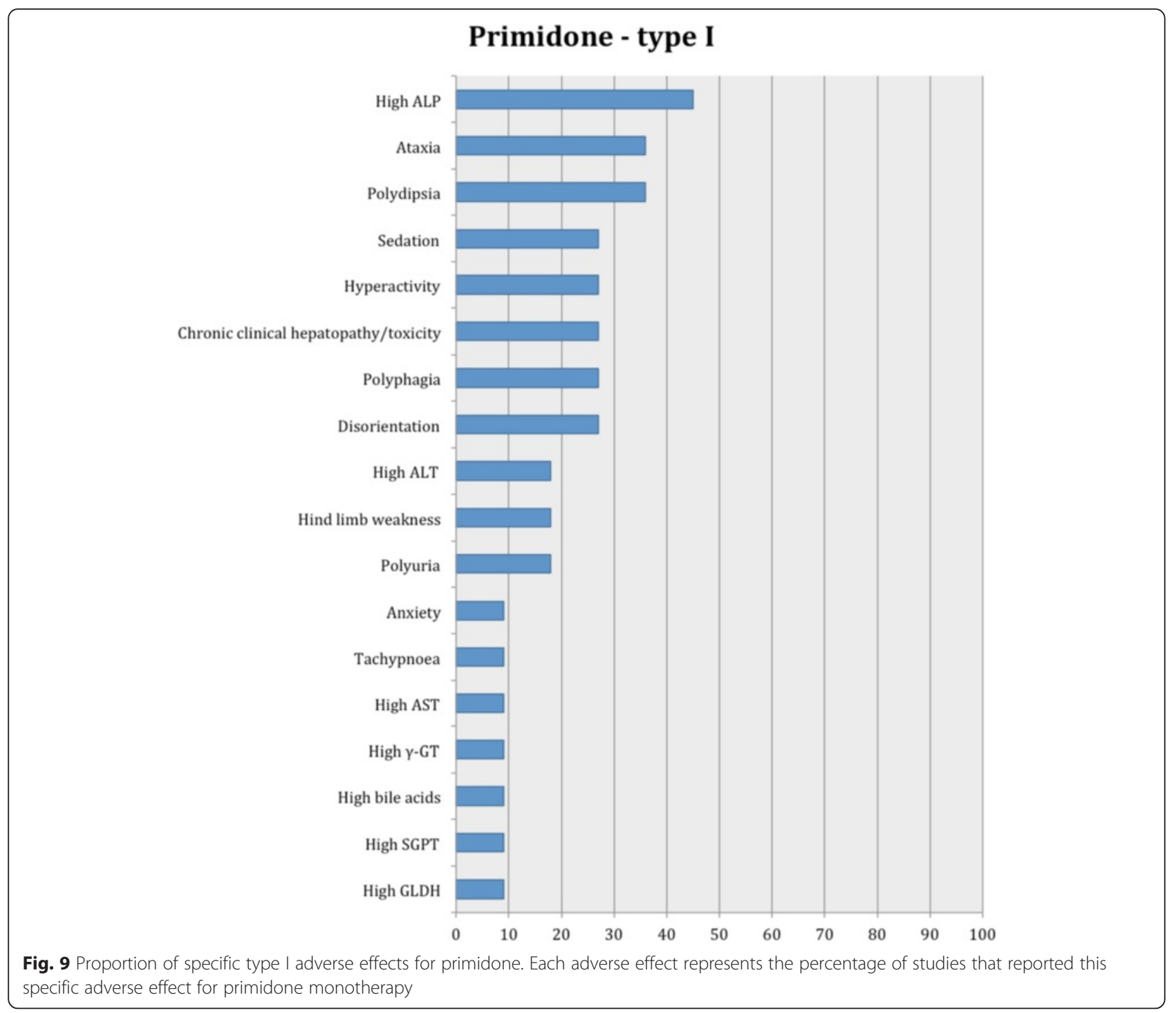

Adequate information about the treatment period was reported in 7/9 (78 \%) (Table 2). From these, in 6/7 $(86 \%)$ the treatment period was relatively short ( $<6$ months). Adequate information about the doses was provided in all studies (Table 2). The imepitoin maintenance doses were mainly at the higher recommended dose, but the toxicology studies in which higher than recommended doses were used. Adequate information about the imepitoin serum levels was reported in $2 / 9$ (22 \%) studies (Table 2), but no correlation was detected between imepitoin serum levels and adverse effects.

\section{Potassium bromide}

There was an overall strong level of evidence provided for potassium bromide safety profile as monotherapy and weak as an adjunctive therapy. Twenty-one studies $[3,23,29,39,51,53,66,67,73,75,77,86,88,93,96$, $101,102,106,110,116]$ presented data about the safety of potassium bromide either as monotherapy (8 studies) $[3,23,29,39,50,51,73,86]$ or adjunctive therapy to phenobarbital and/or other AEDs (16 studies) [3, 39, 50, $53,66,67,75,77,88,93,96,101,102,106,110,116]$, giving a combined sample size of 1940 dogs.

Fifteen studies reported type I adverse effects, including neurological signs as the most common (Table 3). From these, 7/15 (47 \%) and 10/15 (67 \%) studies reported type I adverse effects for potassium bromide monotherapy and adjunctive therapy, respectively. Specifically, in all the studies, the adverse effects most commonly reported were ataxia followed by sedation, polyuria, polydipsia, polyphagia, paraparesis, hyperactivity, vomiting, increased serum ALP and ALT activity. Less commonly, diarrhoea, anorexia, aggression and then tetraparesis, skin issues, euthyroid sick syndrome, anisocoria, chronic clinical hepatopathy/toxicity, and lastly increased serum chloride, bile acids and AST 


\section{Felbamate - type I}

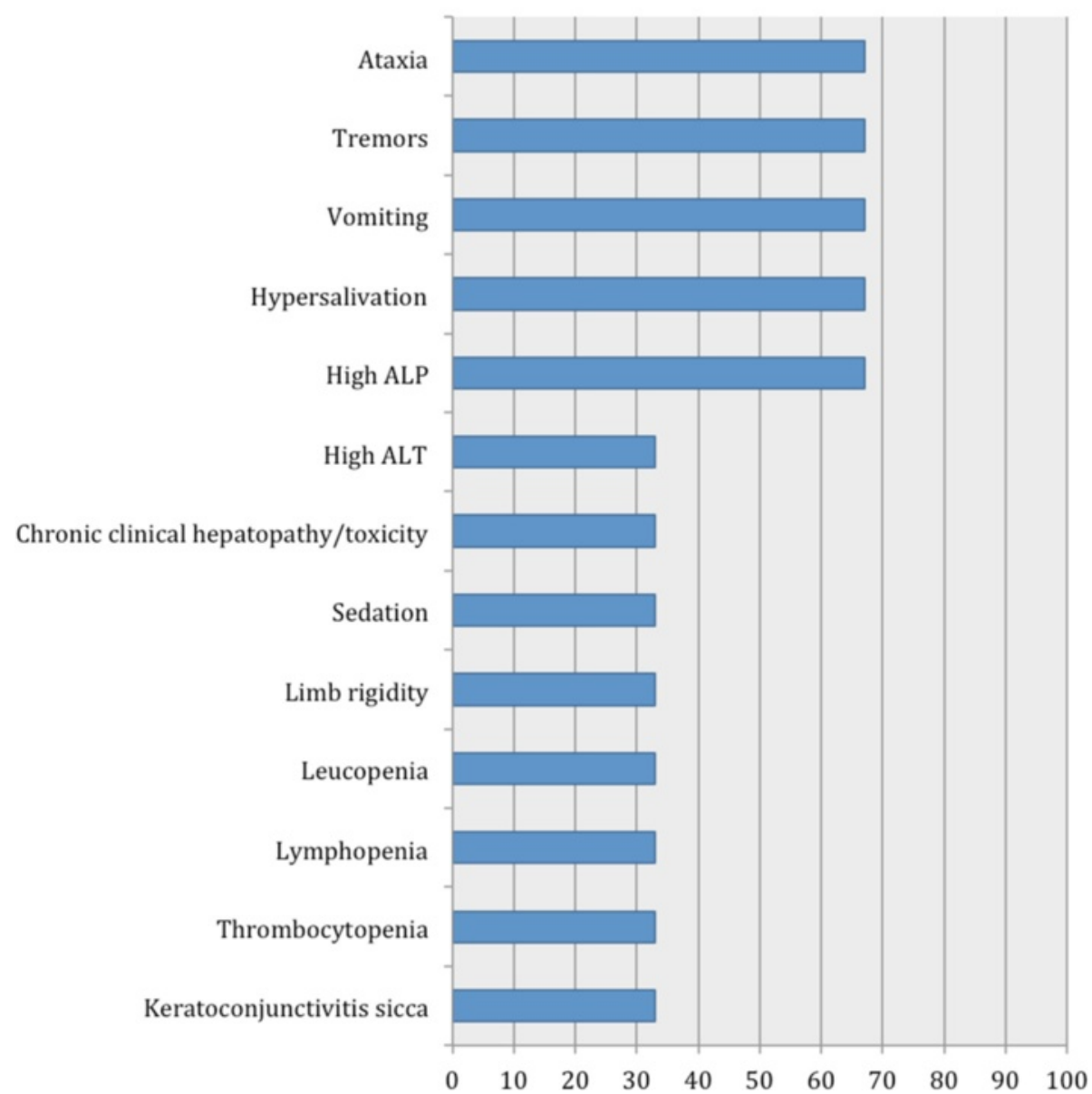

Fig. 10 Proportion of specific type I adverse effects for felbamate. Each adverse effect represents the percentage of studies that reported this specific adverse effect for felbamate monotherapy and/or adjunctive therapy

activity were reported. A similar pattern was shown in the adjunctive therapy studies; diarrhoea, skin conditions and increased amylase and lipase were not reported. In the monotherapy studies, the most common adverse effects were ataxia, sedation and hyperactivity followed by polyuria, polydipsia, polyphagia and diarrhoea; clinical hepatopathy/toxicity, tetraparesis, anisocora, euthyroid sick syndrome, increased serum bile acids and AST activity were not reported (Fig. 5). In one study [51], euthyroid sick syndrome was detected in both potassium bromide-treated and placebo group, indicating that this AED might not affect thyroid function. In another study [39], potassium bromide monotherapy did not affect the thyroxin serum levels.

Nine studies reported type II adverse effects, including gastro-intestinal signs as the most common (Table 3). Specifically, pancreatitis was most commonly reported, followed by panniculitis, generalized appendicular repetitive myoclonus, neuromyopathy with generalized lower motor signs and hyperchloraemia with negative anion gap (Fig. 6). The latter was detected in a dog, two days after a loading dose $(200 \mathrm{mg} / \mathrm{kg}$ BID orally) of potassium bromide was given, but not after regular maintenance doses. In one study [50], although clinical pancreatitis due to potassium bromide or the phenobar$\mathrm{bital} /$ potassium bromide combination was suspected, it was not confirmed as the history and clinical status of the dogs were unknown.

Adequate data to allow calculations of the prevalence of adverse effects was reported in 14/21 (67\%) of the studies (Table 3). From these, 7/15 (47 \%) showed $>50 \%$ prevalence of adverse effect for the specific period of treatment they were conducted. Based on the $95 \% \mathrm{CI}$, the majority of the combined study population experienced at least one adverse effect in 7/15 (47 \%) studies (Table 3).

Adequate information about the treatment period was reported in 17/21 (81 \%) (Table 3). From these, in 5/17 


\section{Phenytoin - type I}

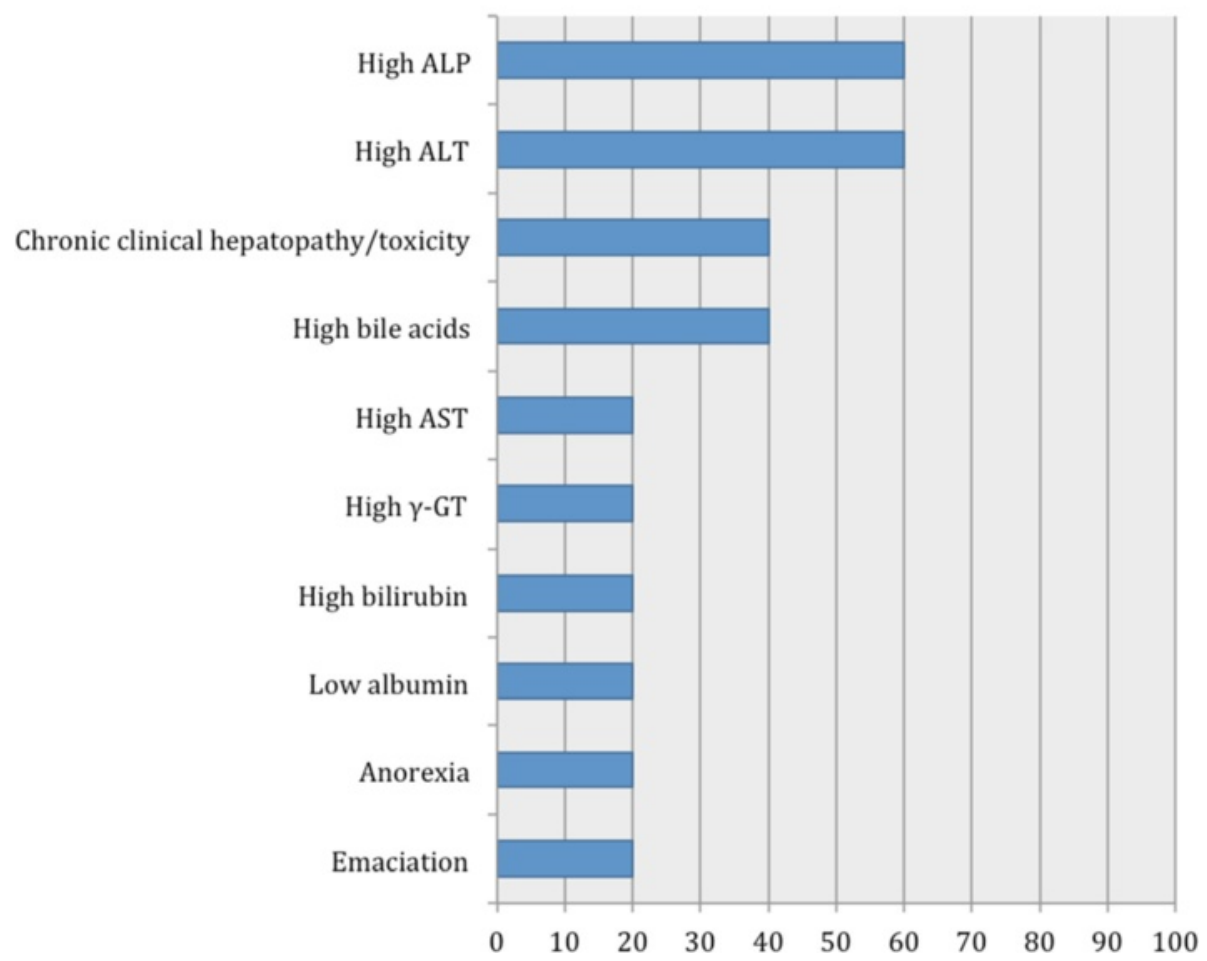

Fig. 11 Proportion of specific type I adverse effects for phenytoin. Each adverse effect represents the percentage of studies that reported this specific adverse effect for phenytoin monotherapy and/or adjunctive therapy

(29 \%) the treatment period was relatively short ( $<6$ months). Adequate information about the doses was provided in 13/21 (62\%) studies (Table 3). From these 9/13 (69 \%) and 4/13 (31 \%) reported doses for type I and type II adverse effects respectively. The maintenance doses were within the recommended dose margins in all but one study. Adequate information about the potassium bromide serum levels was reported in 16/21 (76 \%) studies (Table 3). From these, 12/16 (75 \%) and 6/16 (37 \%) reported serum levels for type I and type II adverse effects respectively. The potassium bromide serum levels were higher than the recommended margins in type I adverse effects (7/12, $58 \%$ ), while within normal ranges for type II adverse effects (4/6, $67 \%)$.

\section{Levetiracetam}

There was an overall strong level of evidence provided for the levetiracetam safety profile as monotherapy and adjunctive therapy. Six studies $[24,27,53,60,72,90]$ presented data about the safety profile of levetiracetam as monotherapy [27, 72] or adjunct to other AEDs (four remaining studies), giving a combined sample size of 129 dogs. One study [53] included both a clinical trial and retrospective case series part.
All the studies reported only type I adverse effects including neurological and gastro-intestinal signs as the most common (Table 4). Specifically, adverse effects most commonly included were vomiting and sedation followed by ataxia and hyperactivity. Less commonly, anorexia, polyphagia, polydipsia followed by polyuria, diarrhoea, aggression, disobedience and attention seeking were reported (Fig. 7). In the monotherapy study, only one episode of vomiting occurred. In one study, no adverse effects were reported.

Adequate data to allow calculations of the prevalence of adverse effects was reported in all studies (Table 4). From these, only two showed $>50 \%$ prevalence of adverse effects for the specific period of treatment during which it was conducted. Based on the $95 \% \mathrm{CI}$, the majority of the population experienced adverse effects in none of the studies (Table 4).

Adequate information about the treatment period was reported in all of the studies (Table 4). From these, in 3/ 7 (43\%) the treatment period was relatively short ( $<6$ months). Adequate information about the doses was provided in all of studies (Table 4). The maintenance doses exceeded the recommended dose range in two studies. Adequate information about the levetiracetam serum levels was reported in 2/7 (29\%) studies (Table 4), 
Table 1 Details of number of dogs, $95 \% \mathrm{Cl}$ affected cases, AED doses and serum levels, treatment period and adverse effects

\begin{tabular}{|c|c|c|c|c|c|c|c|c|c|c|}
\hline Studies & AED & $\begin{array}{l}\text { No of dogs } \\
\text { treated }\end{array}$ & Prevalence & $\begin{array}{l}95 \% \mathrm{Cl} \\
\text { affected cases }\end{array}$ & Doses of AEDs & $\begin{array}{l}\text { Serum levels } \\
\text { of AEDs }\end{array}$ & $\begin{array}{l}\text { Treatment } \\
\text { period }\end{array}$ & $\begin{array}{l}\text { Body system affected and adverse } \\
\text { effects }\end{array}$ & $\begin{array}{l}\text { Most common } \\
\text { adverse effects }\end{array}$ & $\begin{array}{l}\text { Adverse } \\
\text { effect type }\end{array}$ \\
\hline $\begin{array}{l}\text { Boothe et al. } \\
2012\end{array}$ & PHB & 20 & $78.5 \%$ & $60.5 \%-96.5 \%$ & $\begin{array}{l}\text { mean, } 4.11+/-1.1 \\
\text { range, } 3.9-4.9 \\
\mathrm{mg} / \mathrm{kg} \text { PO BID }\end{array}$ & $\begin{array}{l}\text { mean, } 27+/-6 ; \\
\text { range, } 12.4-36 \\
\mu \mathrm{g} / \mathrm{mL}\end{array}$ & $6 \mathrm{~m}$ & $\begin{array}{l}\text { Neurological (ataxia, hyperactivity, } \\
\text { sedation), GI (vomiting, diarrhea, PP), } \\
\text { PU, PD, ClinPath (increased ALP, } \\
\text { decreased albumin) }\end{array}$ & $\begin{array}{l}\text { ataxia, sedation, } \\
\text { increased serum ALP, } \\
\text { decreased albumin }\end{array}$ & 1 \\
\hline $\begin{array}{l}\text { Heynold et al. } \\
1997\end{array}$ & PHB & 37 & $35 \%$ & $19.6 \%-50.4 \%$ & $\begin{array}{l}\text { mean, } 2.5 \mathrm{mg} / \mathrm{kg} \\
\text { PO BID }\end{array}$ & $\begin{array}{l}\text { range, } 15-40 \\
\mu \mathrm{g} / \mathrm{ml}\end{array}$ & $\begin{array}{l}\text { mean, 50; } \\
\text { range, } \\
8-108 \mathrm{~m}\end{array}$ & $\begin{array}{l}\text { Neurological (ataxia, sedation, } \\
\text { aggression), GI (PP), Dermatological } \\
\text { (itching) }\end{array}$ & sedation & 1 \\
\hline $\begin{array}{l}\text { Löscher et al. } \\
2013\end{array}$ & PHB & 8 & NA & NA & $\begin{array}{l}\text { range, } 10-40 \\
\mathrm{mg} / \mathrm{kg} \text { PO SID }\end{array}$ & $14.7 \mu \mathrm{g} / \mathrm{ml}$ & $2.5 \mathrm{~m}$ & $\begin{array}{l}\text { Neurological (sedation, ataxia), } \\
\text { PU, PD }\end{array}$ & $\begin{array}{l}\text { sedation, ataxia, } \\
\text { PU, PD }\end{array}$ & 1 \\
\hline $\begin{array}{l}\text { Gaskill et al. } \\
2000\end{array}$ & PHB & 22 & $32 \%$ & $12.5 \%-51.5 \%$ & $\begin{array}{l}\text { At } 3 \text { w: mean, } \\
3.6+/-1.3 \text {; range, } \\
1.3-6.0 \mathrm{mg} / \mathrm{kg} \\
\text { At } 6 \mathrm{~m} \text { : mean, } \\
3.7+/-1.4 ; \text { range, } \\
1.3-8.3 \mathrm{mg} / \mathrm{kg} \\
\text { At } 12 \mathrm{~m} \text { : } \mathrm{mean}, \\
3.7+/-1.6 ; \text { range, } \\
1.3-8.3 \mathrm{mg} / \mathrm{kg} \\
\text { PO SID }\end{array}$ & $\begin{array}{l}\text { At } 3 \text { w: mean, } \\
58.6+/-15.0 ; \\
\text { range, 33-85 } \\
\text { mmol/L } \\
\text { At } 6 \mathrm{~m}: \\
62.5+/-25.7 ; \\
\text { range, 8-120 } \\
\text { mmol/L. } \\
\text { At } 12 \mathrm{~m} \text { : mean, } \\
62.2+/-23.51 ; \\
\text { range, } 11-116 \\
\mathrm{mmol} / \mathrm{L}\end{array}$ & $12 \mathrm{~m}$ & $\begin{array}{l}\text { Endocrine (decreased total T4 levels, } \\
\text { increased TSH levels, normal TSH } \\
\text { stimulation test) }\end{array}$ & $\begin{array}{l}\text { euthyroid sick } \\
\text { syndrome }\end{array}$ & 1 \\
\hline $\begin{array}{l}\text { Steinberg } \\
2004\end{array}$ & $\begin{array}{l}\text { PHB } \\
\text { (monotherapy } \\
\text { prior to the } \\
\text { addition of } \\
\text { other AEDs) }\end{array}$ & 14 & $26.6 \%$ & $4.8 \%-52.1 \%$ & NA & $\begin{array}{l}\text { PHB: mean, } \\
32.1+/-14.4 \\
\mu \mathrm{g} / \mathrm{ml}\end{array}$ & $\begin{array}{l}\text { median, 17; } \\
\text { range, } \\
3.3-58.5 \mathrm{~m}\end{array}$ & Gl (chronic hepatotoxicity) & $\begin{array}{l}\text { chronic } \\
\text { hepatotoxicity }\end{array}$ & 1 \\
\hline $\begin{array}{l}\text { von Klopmann } \\
\text { et al. } 2006\end{array}$ & PHB & 34 & $68 \%$ & $52.3 \%-83.7 \%$ & NA & NA & NA & $\begin{array}{l}\text { Endocrine (decreased total T4 levels, } \\
\text { normal TSH levels, normal TSH } \\
\text { stimulation test) }\end{array}$ & $\begin{array}{l}\text { euthyroid sick } \\
\text { syndrome }\end{array}$ & 1 \\
\hline $\begin{array}{l}\text { Chang et al. } \\
2006\end{array}$ & PHB & 11 & $92.5 \%$ & $76.9 \%-108.1 \%$ & NA & NA & $\begin{array}{l}\text { median, 18; } \\
\text { range, } \\
3-72 \mathrm{~m}\end{array}$ & $\begin{array}{l}\text { Neurological (ataxia, hyperactivity, } \\
\text { sedation), GI (vomiting, diarrhea, PP), } \\
\text { Dermatological (itching), PU, PD }\end{array}$ & $\begin{array}{l}\mathrm{PD}, \mathrm{PU}, \mathrm{PP} \text {, sedation, } \\
\text { hyperactivity }\end{array}$ & 1 \\
\hline $\begin{array}{l}\text { Tipold et al. } \\
2014\end{array}$ & PHB & 110 & $57.3 \%$ & $48.1 \%-66.5 \%$ & $\begin{array}{l}\text { range, 2-6 } \\
\mathrm{mg} / \mathrm{kg} \text { PO BID }\end{array}$ & $<45 \mu \mathrm{g} / \mathrm{mL}$ & $5 \mathrm{~m}$ & $\begin{array}{l}\text { Neurological (sedation), Gl } \\
\text { (PP, diarrhea), PU, PD, ClinPath } \\
\text { (increased ALP, } \gamma-G T \text {, ALT and GLDH) }\end{array}$ & sedation, PP, PU, PD & 1 \\
\hline $\begin{array}{l}\text { Fredso et al. } \\
2015\end{array}$ & PHB & 6 & $93 \%$ & $70 \%-114 \%$ & $\begin{array}{l}\text { median, } 2.7 ; \\
\text { mean, } 3 ; \text { range } \\
2.2-3 \mathrm{mg} / \mathrm{kg} \\
\mathrm{PO} \mathrm{BID}\end{array}$ & $\begin{array}{l}\text { median, } 77 ; \\
\text { mean, } 77.3 ; \\
\text { range } 55-111 \\
\mu \mathrm{mol} / \mathrm{L}\end{array}$ & $2-12 m$ & $\begin{array}{l}\text { Neurological (sedation, ataxia, } \\
\text { hyperactivity, disobedience), Gl (PP), } \\
\text { PU, PD }\end{array}$ & $P D, P P$ & 1 \\
\hline $\begin{array}{l}\text { Schwartz- } \\
\text { Porsche et al. } \\
1985\end{array}$ & PHB & 15 & $93 \%$ & $80.1 \%-105.9 \%$ & $\begin{array}{l}\text { range, 5-17 } \\
\mathrm{mg} / \mathrm{kg} \text { PO SID }\end{array}$ & $\begin{array}{l}\text { range, } \\
19-57 \mu \mathrm{g} / \mathrm{ml}\end{array}$ & $\begin{array}{l}\text { mean, } 15 ; \\
\text { range, } \\
7.3-32 \mathrm{~m}\end{array}$ & $\begin{array}{l}\text { Neurological (sedation, ataxia), Gl } \\
(\mathrm{PP}), \mathrm{PD}, \mathrm{ClinPath}(\mathrm{ALT}, \mathrm{ALP}, \mathrm{GLDH})\end{array}$ & $\begin{array}{l}\text { ataxia, sedation, } \\
\text { PP, PD }\end{array}$ & 1 \\
\hline $\begin{array}{l}\text { Gaskill et al. } \\
2005\end{array}$ & PHB & 12 & NA & NA & $\begin{array}{l}\text { median, 5; range, } \\
2.1-12.9 \mathrm{mg} / \mathrm{kg} \\
\text { PO SID }\end{array}$ & $\begin{array}{l}\text { mean, 22.8; } \\
\text { range, } 9.7-44.2 \\
\mu \mathrm{g} / \mathrm{ml}\end{array}$ & $\begin{array}{l}\text { median, } 20.4 ; \\
\text { range, } 4-78 \mathrm{~m}\end{array}$ & ClinPath (increased ALT, ALP) & increased ALT, ALP & 1 \\
\hline
\end{tabular}


Table 1 Details of number of dogs, $95 \% \mathrm{Cl}$ affected cases, AED doses and serum levels, treatment period and adverse effects (Continued)

\begin{tabular}{|c|c|c|c|c|c|c|c|c|c|c|}
\hline $\begin{array}{l}\text { Farnbach et al. } \\
1984\end{array}$ & PHB & 42 & $2.4 \%$ & $-2.2 \%-7.2 \%$ & $\begin{array}{l}\text { range, } 0.3-19.9 \\
\mathrm{mg} / \mathrm{kg} \text { PO SID }\end{array}$ & $\begin{array}{l}\text { mean, 24.3; } \\
\text { range, 6.5-81.3 } \\
\mu \mathrm{g} / \mathrm{ml}\end{array}$ & NA & Neurological (hyperactivity) & hyperactivity & 1 \\
\hline $\begin{array}{l}\text { Gaskill and } \\
\text { Kimber } 2010\end{array}$ & PHB & 30 & $80 \%$ & $65.7 \%-94.3 \%$ & NA & NA & $12 \mathrm{~m}$ & $\begin{array}{l}\text { Neurological (ataxia, sedation, } \\
\text { hyperactivity, aggression), Gl } \\
\text { (PP, anorexia, vomiting, diarrhoea), } \\
\text { Dermatological (skin problems), } \\
\text { ClinPath (increased ALP, ALT, lipase), } \\
\text { PU, PD }\end{array}$ & $\begin{array}{l}\text { PP, PU, PD, vomiting, } \\
\text { skin problems, } \\
\text { hyperactivity }\end{array}$ & 1 \\
\hline $\begin{array}{l}\text { Aitken et al. } \\
2003\end{array}$ & PHB & 95 & $40 \%$ & $30.1 \%-49.8 \%$ & $\begin{array}{l}<2->10 \mathrm{mg} / \mathrm{kg} \\
\text { PO SID }\end{array}$ & $<65->120 \mu \mathrm{mol} / \mathrm{l}$ & $<3->12 \mathrm{~m}$ & $\begin{array}{l}\text { ClinPath (increased ALT, ALP, } Y-G T \text {, } \\
\text { GLDH, cholesterol, bile acids) }\end{array}$ & $\begin{array}{l}\text { increased ALP, } \\
\text { ALT, GLDH }\end{array}$ & 1 \\
\hline $\begin{array}{l}\text { Dayrell-Hart } \\
\text { et al. } 1991\end{array}$ & PHB & 18 & NA & NA & $\begin{array}{l}\text { median, } 10.4 ; \\
\text { range, } 3.1-27 \\
\mathrm{mg} / \mathrm{kg} \text { PO SID }\end{array}$ & $\begin{array}{l}\text { mean, } 49.7 ; \\
\text { range, } 16-60 \\
\mu \mathrm{g} / \mathrm{ml}(12 \text { dogs } \\
\text { had }>40)\end{array}$ & $\begin{array}{l}\text { median, 39; } \\
\text { range, 5-82 } \mathrm{m}\end{array}$ & $\begin{array}{l}\text { Gl (hepatotoxicity) } \\
\text { (also all dogs were ataxic and } \\
\text { sedated) }\end{array}$ & NA & I \\
\hline $\begin{array}{l}\text { Andrik et al. } \\
2010\end{array}$ & PHB & $\begin{array}{l}30 \\
\text { (15 epileptic } \\
\text { and } 15 \\
\text { non-epileptic) }\end{array}$ & NA & NA & $\begin{array}{l}\text { Epileptic dogs: } \\
2 \mathrm{mg} / \mathrm{kg} \text { PO BID } \\
\text { (increased if } \\
\text { necessary) } \\
\text { Non-epileptic } \\
\text { dogs: Initially at } \\
2 \mathrm{mg} / \mathrm{kg} \text { PO SID, } \\
\text { then increased at } \\
8 \mathrm{mg} / \mathrm{kg} \text { PO SID }\end{array}$ & NA & $\begin{array}{l}\text { Epileptic dogs: } \\
\text { range, } 12-60 \mathrm{~m} \\
\text { Non-epileptic } \\
\text { dogs: } 5 \mathrm{~m}\end{array}$ & $\begin{array}{l}\text { Gl (chronic hepatotoxicity), ClinPath } \\
\text { (increased ALP, ALT, AST, total } \\
\text { bilirubin, decreased albumin and } \\
\text { total protein) }\end{array}$ & $\begin{array}{l}\text { increased ALT, } \\
\text { ALP }\end{array}$ & I \\
\hline $\begin{array}{l}\text { Litchfield et al. } \\
1972\end{array}$ & PHB & 4 & NA & NA & $\begin{array}{l}\text { range, 5-40 } \\
\mathrm{mg} / \mathrm{kg} \text { IV SID }\end{array}$ & NA & $0.5 \mathrm{~m}$ & ClinPath (increased ALP) & NA & I \\
\hline $\begin{array}{l}\text { Foster et al. } \\
2000\end{array}$ & PHB & $\begin{array}{l}\text { Experimental } \\
\text { dogs: } 6 \\
\text { Epileptic } \\
\text { dogs: } 10\end{array}$ & $70 \%$ & $\begin{array}{l}\text { Experimental } \\
\text { dogs: } 0 \% \\
\text { Epileptic dogs: } \\
41.6 \%-98.4 \%\end{array}$ & $\begin{array}{l}\text { Experimental } \\
\text { dogs: mean, } \\
6 \mathrm{mg} / \mathrm{kg} ; \mathrm{range}, \\
5.9-6.4 \mathrm{mg} / \mathrm{kg} \\
\text { PO SID } \\
\text { Epileptic dogs: } \\
\text { range, 3.9-14.4 } \\
\mathrm{mg} / \mathrm{kg} \text { PO SID }\end{array}$ & $\begin{array}{l}\text { Experimental } \\
\text { dogs: mean, } \\
63+/-15 \text {, range, } \\
<65-194 \mu \mathrm{mol} / \mathrm{L} \\
\text { Epileptic dogs: } \\
\text { mean, 110; } \\
\text { range, 72-171 } \\
\mu \mathrm{mol} / \mathrm{L}\end{array}$ & $\begin{array}{l}\text { Experimental } \\
\text { dogs: } 3 \mathrm{~m} \\
\text { Epileptic dogs: } \\
\text { range, } 14-92 \mathrm{~m}\end{array}$ & ClinPath (increased ALP, ALT, cholesterol) & increased ALP & 1 \\
\hline $\begin{array}{l}\text { Gaskil et al. } \\
1999\end{array}$ & PHB & 78 & $40 \%$ & $28.8 \%-50.6 \%$ & $\begin{array}{l}\text { median 4; range, } \\
1-16.4 \mathrm{mg} / \mathrm{kg} \\
\text { PO SID }\end{array}$ & $\begin{array}{l}\text { median, 17.6; } \\
\mathrm{range}, 4-70 \\
\mu \mathrm{g} / \mathrm{ml}\end{array}$ & $\begin{array}{l}\text { median, 12.5; } \\
\text { range, 0.3-96 }\end{array}$ & $\begin{array}{l}\text { Endocrine (decreased total T4, free } \\
\text { T4, increased TSH) } \\
\text { Also, ClinPath abnormalities were } \\
\text { reported, i.e. increased ALT, ALP, } \\
\text { AST, } \gamma \text {-GT, fasting bile acids and } \\
\text { cholesterol, but no further details } \\
\text { are provided. }\end{array}$ & $\begin{array}{l}\text { eythyroid sick } \\
\text { syndrome }\end{array}$ & 1 \\
\hline $\begin{array}{l}\text { Muller et al. } \\
2000\end{array}$ & PHB & 12 & $91.6 \%$ & $\begin{array}{l}100 \% \text { or } \\
50.5 \%-99.5 \%\end{array}$ & $\begin{array}{l}\text { mean, } 5 ; \text { range, } \\
4.8-6.6 \mathrm{mg} / \mathrm{kg} \\
\text { PO BID }\end{array}$ & $\begin{array}{l}\text { range, } \\
20-40 \mu \mathrm{g} / \mathrm{mL}\end{array}$ & $7.1 \mathrm{~m}$ & $\begin{array}{l}\text { Endocrine (decreased total T4, free } \\
\text { T4, increased TSH, cholesterol and } \\
\text { total T3), Neurological (sedation for } \\
\text { the first } 3 \text { days) } \\
\text { No significant PHB's effect on either } \\
\text { of the adrenal function tests }\end{array}$ & $\begin{array}{l}\text { euthyroid sick } \\
\text { syndrome }\end{array}$ & I \\
\hline
\end{tabular}


Table 1 Details of number of dogs, $95 \% \mathrm{Cl}$ affected cases, AED doses and serum levels, treatment period and adverse effects (Continued)

\begin{tabular}{|c|c|c|c|c|c|c|c|c|c|c|}
\hline $\begin{array}{l}\text { Muller et al. } \\
2000\end{array}$ & PHB & 12 & $87.5 \%$ & $75.9 \%-107.3 \%$ & $\begin{array}{l}\text { mean, } 5 \text {; range, } \\
4.8-6.6 \mathrm{mg} / \mathrm{kg} \\
\text { PO BID }\end{array}$ & $\begin{array}{l}\text { range, } \\
20-40 \mu \mathrm{g} / \mathrm{ml}\end{array}$ & $7.1 \mathrm{~m}$ & $\begin{array}{l}\text { ClinPath (increased ALP, ALT, } \gamma \text {-GT, } \\
\text { decreased albumin), Neurological } \\
\text { (sedation for the first } 3 \text { days) }\end{array}$ & $\begin{array}{l}\text { increased ALP, } \\
\text { ALT, } Y-G T\end{array}$ & I \\
\hline $\begin{array}{l}\text { Kantrowitz } \\
\text { et al. } 1999\end{array}$ & PHB & 55 & NA & NA & NA & $\begin{array}{l}\text { median, } 25.3 ; \\
\text { range, } 8.0-74.3 \\
\mu \mathrm{g} / \mathrm{ml}\end{array}$ & $\begin{array}{l}\text { median, } 7 \mathrm{~m} ; \\
\text { range, } 1-120 \mathrm{~m}\end{array}$ & $\begin{array}{l}\text { Endocrine (decreased T4, } \\
\text { increased TSH) }\end{array}$ & $\begin{array}{l}\text { eythyroid sick } \\
\text { syndrome }\end{array}$ & 1 \\
\hline $\begin{array}{l}\text { Chauvet et al. } \\
1995\end{array}$ & PHB & 5 & $100 \%$ & $100 \%$ & NA & $\begin{array}{l}\text { range, } \\
20-47 \mu \mathrm{g} / \mathrm{ml}\end{array}$ & $13 \mathrm{~m}$ & $\begin{array}{l}\text { Endocrine (increased ACTH, altered } \\
\text { ACTH stimulation and } \\
\text { dexamethasone supression test), } \\
\text { ClinPath (increased ALT, ALP, } \\
\text { decreased albumin, cholesterol), } \\
\text { PU, PD }\end{array}$ & $\begin{array}{l}\text { increased ACTH, } \\
\text { altered ACTH } \\
\text { stimulation and } \\
\text { dexamethasone } \\
\text { supression test, } \\
\text { increased ALT, ALP, } \\
\text { decreased albumin, } \\
\text { cholesterol }\end{array}$ & 1 \\
\hline $\begin{array}{l}\text { Balazs et al. } \\
1978\end{array}$ & PHB & 4 & $100 \%$ & $100 \%$ & $\begin{array}{l}40 \mathrm{mg} / \mathrm{kg} \\
\mathrm{PO} \mathrm{SID}\end{array}$ & NA & $1.8 \mathrm{~m}$ & ClinPath (increased ALP) & increased ALP & 1 \\
\hline $\begin{array}{l}\text { Conning and } \\
\text { Litchfield } 1971\end{array}$ & PHB & NA & NA & NA & NA & NA & NA & ClinPath (increased ALP) & increased ALP & I \\
\hline $\begin{array}{l}\text { Sturtevant } \\
\text { et al. } 1977\end{array}$ & PHB & 2 & $100 \%$ & $100 \%$ & $4.4 \mathrm{mg} / \mathrm{kg}$ PO TID & NA & $1 \mathrm{~m}$ & ClinPath (Increased ALT, ALP) & $\begin{array}{l}\text { increased ALT } \\
\text { and ALP }\end{array}$ & 1 \\
\hline $\begin{array}{l}\text { Thrift et al. } \\
2010\end{array}$ & PHB & 1 & NA & NA & $6.4 \mathrm{mg} / \mathrm{kg} \mathrm{PO} \mathrm{BID}$ & NA & $2 m$ & $\begin{array}{l}\text { ClinPath (anemia, increased } \\
\text { ALT, ALP, AST) }\end{array}$ & idiosyncrasic anemia & |\& || \\
\hline $\begin{array}{l}\text { Kube et al. } \\
2006\end{array}$ & PHB & 1 & NA & NA & $\begin{array}{l}\text { Initially } 5 \mathrm{mg} / \mathrm{kg} \\
\text { PO BID for } 4 \text { days, } \\
\text { then } 3 \mathrm{mg} / \mathrm{kg} \\
\text { PO BID }\end{array}$ & NA & $2 m$ & Dyskinesia (twitching episodes) & NA & $\|$ \\
\hline $\begin{array}{l}\text { Steiner et al. } \\
2008\end{array}$ & PHB & 118 & $14.4 \%$ & $8.1 \%-20.7 \%$ & NA & Unclear & NA & ClinPath (Increased cPLI) & NA & $\|$ \\
\hline $\begin{array}{l}\text { Gaskill et al. } \\
2000\end{array}$ & PHB & 88 & $9 \%$ & $3.0 \%-15.0 \%$ & NA & $\begin{array}{l}\text { range, } \\
39-130 \mathrm{~mol} / \mathrm{L}\end{array}$ & $16 \mathrm{~m}$ & $\begin{array}{l}\text { GI (pancreatitis, increased amylase } \\
\text { and/or lipase activities) }\end{array}$ & $\begin{array}{l}\text { increased amylase } \\
\text { and/or lipase activities }\end{array}$ & $\|$ \\
\hline $\begin{array}{l}\text { March et al. } \\
2004\end{array}$ & PHB & 11 & NA & NA & $\begin{array}{l}\text { mean, } 12.4+/-5.7 \\
\text { range, 3.8-19.8 } \\
\mathrm{mg} / \mathrm{kg} \text { PO SID }\end{array}$ & $\begin{array}{l}\text { mean, } \\
43.5+/-15.1 ; \\
\text { range, } 22.8-66 \\
\mu \mathrm{g} / \mathrm{ml}\end{array}$ & $\begin{array}{l}\text { median, 6; range, } \\
\text { 20.4-132 } \mathrm{m}\end{array}$ & $\begin{array}{l}\text { Dermatological (superficial } \\
\text { necrolytic dermatitis) }\end{array}$ & NA & $\|$ \\
\hline Weiss 2005 & PHB & 3 & NA & NA & NA & NA & NA & $\begin{array}{l}\text { Blood dyscrasias (bone marrow } \\
\text { necrosis-myelofibrosis) }\end{array}$ & NA & $\|$ \\
\hline $\begin{array}{l}\text { Jacobs et al. } \\
1998\end{array}$ & PHB & 2 & NA & NA & $\begin{array}{l}\text { Case 1: } 2.2 \mathrm{mg} / \mathrm{kg} \\
\text { PO BID; Case 2: } \\
4.4 \mathrm{mg} / \mathrm{kg} \text { PO BID }\end{array}$ & NA & Case 1: 5 m; Case 2: $3 \mathrm{~m}$ & $\begin{array}{l}\text { Blood dyscrasias (neutropenia, } \\
\text { thrombocytopenia), ClinPath } \\
\text { (increase ALP) }\end{array}$ & NA & $\|$ \\
\hline $\begin{array}{l}\text { Weiss et al. } \\
2002\end{array}$ & PHB & 1 & NA & NA & NA & NA & NA & Blood dyscrasias (myelofibrosis) & NA & $\|$ \\
\hline $\begin{array}{l}\text { Bevier et al. } \\
2010\end{array}$ & PHB & 1 & NA & NA & NA & NA & NA & $\begin{array}{l}\text { Dermatological } \\
\text { (superficial necrolytic dermatitis) }\end{array}$ & NA & $\|$ \\
\hline
\end{tabular}


Table 1 Details of number of dogs, $95 \% \mathrm{Cl}$ affected cases, AED doses and serum levels, treatment period and adverse effects (Continued)

\begin{tabular}{|c|c|c|c|c|c|c|c|c|c|c|}
\hline $\begin{array}{l}\text { Bersan et al. } \\
2014\end{array}$ & PHB & 16 & NA & NA & $\begin{array}{l}\text { median, 3; mean, } \\
2.75+/-0.43 \text {; range, } \\
1.60-7.25 \mathrm{mg} / \mathrm{kg} \\
\text { PO BID }\end{array}$ & $\begin{array}{l}\text { median, 19; } \\
\text { mean, 22.4+/-5.5; } \\
\text { range, 13.2-30.5 } \\
\mu \mathrm{g} / \mathrm{ml}\end{array}$ & $\begin{array}{l}\text { median, 69.5; } \\
\text { mean, } \\
72.1+/- \text { sd 45.8; } \\
\text { range, } \\
14-157 \mathrm{~m}\end{array}$ & $\begin{array}{l}\text { Blood dyscrasias (anemia and/or } \\
\text { thrombocytopenia and/or } \\
\text { neutropenia and/or pancytopenia) }\end{array}$ & anemia, pancytopenia & $\|$ \\
\hline $\begin{array}{l}\text { Volk et al. } \\
2008 \\
\text { (case series) }\end{array}$ & $\begin{array}{l}\text { PHB } \\
\text { (monotherapy } \\
\text { prior to the } \\
\text { addition of } \\
\text { other AEDs) }\end{array}$ & 8 & NA & NA & $\begin{array}{l}\text { NA but was within } \\
\text { normal reference } \\
\text { values }\end{array}$ & NA & $\begin{array}{l}\text { Approximately } \\
2-3 \mathrm{~m}\end{array}$ & $\begin{array}{l}\text { Blood dyscrasias } \\
\text { (bone marrow suppression) }\end{array}$ & NA & $\|$ \\
\hline $\begin{array}{l}\text { Habock and } \\
\text { Pakozdy } 2012\end{array}$ & PHB & 37 & $22 \%$ & $16.8 \%-57.2 \%$ & NA & NA & $>1 \mathrm{~m}$ & $\begin{array}{l}\text { Blood dyscrasias (anemia and/or } \\
\text { thrombocytopenia and/or } \\
\text { neutropenia and/or pancytopenia) }\end{array}$ & NA & $\|$ \\
\hline $\begin{array}{l}\text { Von Klopmann } \\
\text { et al. } 2006\end{array}$ & PHB & 1 & NA & NA & $2 \mathrm{mg} / \mathrm{kg} \mathrm{PO} \mathrm{BID}$ & NA & & Blood dyscrasias (pancytopenia) & NA & $\|$ \\
\hline $\begin{array}{l}\text { Bizzeti et al. } \\
2006\end{array}$ & PHB & 7 & $14.4 \%$ & $-11.6 \%-40.2 \%$ & NA & NA & NA & $\begin{array}{l}\text { Pancreatitis, ClinPath } \\
\text { (Increased amylase, lipase, cPLI) }\end{array}$ & NA & $\|$ \\
\hline $\begin{array}{l}\text { Mathis et al. } \\
2014\end{array}$ & PHB & 1 & NA & NA & $2.1 \mathrm{mg} / \mathrm{kg} \mathrm{PO} B \mathrm{BI}$ & $27.5 \mu \mathrm{g} / \mathrm{dL}$ & $6 \mathrm{~m}$ & $\begin{array}{l}\text { Blood dyscrasias } \\
\text { (bone marrow supression) }\end{array}$ & NA & $\|$ \\
\hline $\begin{array}{l}\text { Daminet et al. } \\
1999\end{array}$ & PHB & 9 & $0 \%$ & $0 \%$ & $\begin{array}{l}\text { Initially } 1.8-3 \text { for } \\
\text { one week, then } \\
2.7-4.5 \mathrm{mg} / \mathrm{kg} \\
\text { PO BID }\end{array}$ & $\begin{array}{l}\text { range, } 65-150 \\
\mathrm{pmol} / \mathrm{L}\end{array}$ & $0.8 \mathrm{~m}$ & No adverse effects & NA & NA \\
\hline $\begin{array}{l}\text { Dyer et al. } \\
1994\end{array}$ & PHB & 6 & $0 \%$ & $0 \%$ & $5 \mathrm{mg} / \mathrm{kg} \mathrm{PO} \mathrm{BID}$ & $\begin{array}{l}\text { range, } 18-37 \\
\mu \mathrm{g} / \mathrm{ml}\end{array}$ & $2 \mathrm{~m}$ & $\begin{array}{l}\text { No PHB's effect on endogenous } \\
\text { ACTH and ACTH stimulation test }\end{array}$ & NA & NA \\
\hline
\end{tabular}

Abbreviations: AED(s) anti-epileptic drug(s), BID bis in die (twice daily), Chloraz Chlorazepate, CSF cerebrospinal fluid, CL confidence level, Gaba Gabapentin, IE idiopathic epilepsy, LEV Levetiracetam, $m$ month(s), NA Not Available, PHB phenobarbital, $P D$ polydipsia, $P U$ polyuria, $P P$ polyphagia, $P B r$ potassium bromide, Prim primidone, $P O$ per os, SID semel in die (once daily), $T I D$ ter in die (three times daily), TPM topiramate, $W$ week(s), $y$ year(s) 
Table 2 Details of number of dogs, $95 \% \mathrm{Cl}$ affected cases, AED doses and serum levels, treatment period and adverse effects

\begin{tabular}{|c|c|c|c|c|c|c|c|c|c|c|}
\hline Studies & AED & $\begin{array}{l}\text { No of dogs } \\
\text { treated }\end{array}$ & Prevalence & $\begin{array}{l}95 \% \mathrm{Cl} \\
\text { affected cases }\end{array}$ & Doses of AEDs & $\begin{array}{l}\text { Serum levels } \\
\text { of AEDs }\end{array}$ & $\begin{array}{l}\text { Treatment } \\
\text { period }\end{array}$ & $\begin{array}{l}\text { Body system affected and } \\
\text { adverse effects }\end{array}$ & $\begin{array}{l}\text { Most common } \\
\text { adverse effects }\end{array}$ & $\begin{array}{l}\text { Adverse } \\
\text { effect type }\end{array}$ \\
\hline $\begin{array}{l}\text { Rundfeldt et } \mathrm{sl} . \\
2015\end{array}$ & Imepitoin & $\begin{array}{l}127 \\
\text { Imepitoin } \\
\text { high dose } \\
\text { group: } 66 \\
\text { Imepitoin } \\
\text { low dose } \\
\text { group: } 61\end{array}$ & $\begin{array}{l}\text { Imepitoin high dose } \\
\text { group: } 86 \% \\
\text { Imepitoin low dose } \\
\text { group: } 82 \%\end{array}$ & $\begin{array}{l}\text { Imepitoin high } \\
\text { dose group: } \\
77.6 \%-94.3 \% \\
\text { Imepitoin low } \\
\text { dose group: } \\
72.3 \%-91.6 \%\end{array}$ & $\begin{array}{l}\text { Imepitoin high } \\
\text { dose group: } \\
30 \mathrm{mg} / \mathrm{kg} \text { PO BID } \\
\text { Imepitoin low } \\
\text { dose group: } \\
1 \text { mg/kg PO BID }\end{array}$ & NA & $\begin{array}{l}\text { 1st phase: } 3 \mathrm{~m} \\
\text { 2nd phase: } 3 \mathrm{~m}\end{array}$ & $\begin{array}{l}\text { Neurological (hyperactivity, } \\
\text { disorientation), musculoskeletal } \\
\text { (unspecified), gastro-intestinal } \\
\text { (unspecified), respiratory } \\
\text { (unspecified), rogenital } \\
\text { (unspecified), other systems } \\
\text { (unspecified), general } \\
\text { (unspecified) }\end{array}$ & $\begin{array}{l}\text { Disorientation, } \\
\text { hyperactivity }\end{array}$ & 1 \\
\hline $\begin{array}{l}\text { Tipold et al. } \\
2014\end{array}$ & Imepitoin & 116 & $46.6 \%$ & $37.5 \%-55.7 \%$ & $\begin{array}{l}10-30 \mathrm{mg} / \mathrm{kg} \\
\text { PO BID }\end{array}$ & NA & $5 \mathrm{~m}$ & $\begin{array}{l}\text { Neurological (sedation, } \\
\text { hyperactivity), Gl (PP, diarrhoea), } \\
\text { PU, PD, Renal//rinary disorders, } \\
\text { ClinPath (increased creatinine) }\end{array}$ & $\begin{array}{l}\text { PP, PD, PU, sedation, } \\
\text { hyperactivity }\end{array}$ & 1 \\
\hline $\begin{array}{l}\text { Tipold et al. } \\
2014 \text { (ELAS) }\end{array}$ & Imepitoin & 32 & NA & NA & $\begin{array}{l}30,90 \text { or } 150 \\
\mathrm{mg} / \mathrm{kg} \text { PO BID } \\
\text { (adverse effects } \\
\text { occurred mainly } \\
\text { in the higest } \\
\text { doses, i.e. } 3 X \\
\text { and } 5 X \text { the } \\
\text { recommended } \\
\text { dose) }\end{array}$ & NA & $6 \mathrm{~m}$ & $\begin{array}{l}\text { Neurological (loss of righting } \\
\text { reflex, ataxia, intermittent } \\
\text { tremors, decreased activity, } \\
\text { nystagmus), Gl (vomiting, } \\
\text { hypersalivation, white material } \\
\text { in the faeces), ClinPath } \\
\text { (increased creatinine), } \\
\text { Ophtalmological (lacrimation, } \\
\text { eye dryness, eye discharges, } \\
\text { relaxed nictitating membranes, } \\
\text { eyelid closure) }\end{array}$ & $\begin{array}{l}\text { NA (infrequent } \\
\text { adverse effects) }\end{array}$ & 1 \\
\hline $\begin{array}{l}\text { Loscher et al. } \\
\text { 2004, Rieck } \\
\text { et al. } 2006\end{array}$ & $\begin{array}{l}\text { Imepitoin as } \\
\text { monotherapy } \\
\text { (12 dogs) and } \\
\text { imepitoin as an } \\
\text { adjunct to PHB } \\
\text { or Primidone } \\
\text { (17 dogs) }\end{array}$ & 29 & $58.6 \%$ & $40.7 \%-76.5 \%$ & $\begin{array}{l}\text { Imepitoin: Initially } \\
5 \mathrm{mg} / \mathrm{kg} \text { PO BID } \\
\text { for } 1 \text { week, then } \\
10-30 \mathrm{mg} / \mathrm{kg} \\
\text { PO BID. } \\
\text { PHB: 6-23 mg/kg } \\
\text { PO SID. } \\
\text { Primidone: } \\
25-53 \mathrm{mg} / \mathrm{kg} \\
\text { PO SID }\end{array}$ & $\begin{array}{l}\text { Imepitoin: mean, } \\
4,000 ; \text { range, } \\
3400-7300 \mathrm{ng} / \mathrm{ml} \\
(2 \mathrm{~h} \text { after dosing) } \\
\text { and mean, } 650 \\
\mathrm{ng} / \mathrm{ml} \text { ( } 12 \mathrm{~h} \\
\text { after dosing). } \\
\text { PHB: range, } \\
15-45 \mu \mathrm{\mu g} / \mathrm{ml} \\
(2 \text { dogs with } \\
\text { adverse effects } \\
\text { had } 56.6-58.9 \\
\text { lg/mL). } \\
\text { Prim: NA }\end{array}$ & $\begin{array}{l}\text { mean, } \\
7.7 \pm 0.7 \mathrm{~m}\end{array}$ & $\begin{array}{l}\text { Neurological (ataxia, sedation), } \\
\text { Gl (PP), ClinPath (increased } \\
\text { ALT, ALP, GLDH) }\end{array}$ & PP & 1 \\
\hline $\begin{array}{l}\text { Löscher et al. } \\
2004 \text { (ELAS) }\end{array}$ & Imepitoin & $\begin{array}{l}\text { 1st } \\
\text { experiment: } 6 \\
\text { 2nd } \\
\text { experiment: } 6\end{array}$ & $0 \%$ & $0 \%$ & $\begin{array}{l}\text { 1st experiment: } \\
5 \mathrm{mg} / \mathrm{kg} \text { PO BID } \\
\text { 2nd experiment: } \\
40 \mathrm{mg} / \mathrm{kg} \text { PO BID }\end{array}$ & $\begin{array}{l}\text { 1st experiment: } \\
\text { range, } 20-120 \\
\mathrm{ng} / \mathrm{ml} \\
\text { 2nd experiment: } \\
\text { range, } \\
4800-7400 \mathrm{ng} / \mathrm{ml}\end{array}$ & $\begin{array}{l}1 \mathrm{st} \text { experiment: } \\
1.2 \mathrm{~m} \\
\text { 2nd experiment: } \\
1.2 \mathrm{~m}\end{array}$ & $\begin{array}{l}\text { 1st experiment: none } \\
\text { 2nd experiment: none but } \\
\text { increase in body weight }\end{array}$ & NA & 1 \\
\hline $\begin{array}{l}\text { EMA report } \\
2012 \\
\text { (US field trial) }\end{array}$ & Imepitoin & 110 & NA & NA & $\begin{array}{l}\text { range, } \\
10-30 \mathrm{mg} / \mathrm{kg} \\
\mathrm{PO} \mathrm{BID}\end{array}$ & NA & NA & $\begin{array}{l}\text { Neurological (ataxia, hyperactivity, } \\
\text { anxiety, disorientation), ClinPath } \\
\text { (increased enzymes-unclear which) } \\
\text { tachypnoea, PD }\end{array}$ & $\begin{array}{l}\text { ataxia, hyperactivity, } \\
\text { anxiety, PD, increased } \\
\text { liver enzymes }\end{array}$ & 1 \\
\hline
\end{tabular}


Table 2 Details of number of dogs, $95 \% \mathrm{Cl}$ affected cases, AED doses and serum levels, treatment period and adverse effects (Continued)

\begin{tabular}{|c|c|c|c|c|c|c|c|c|c|c|}
\hline $\begin{array}{l}\text { EMA report } \\
2012 \\
\text { (unpublished } \\
\text { clinical trials: } \\
\text { Tipold 2006; } \\
\text { Heit 2011; } \\
\text { de Vries 2011) }\end{array}$ & Imepitoin & NA & NA & NA & $\begin{array}{l}30 \mathrm{mg} / \mathrm{kg} \text { PO BID } \\
\text { (Unclear if other } \\
\text { doses were } \\
\text { also used) }\end{array}$ & NA & NA & $\begin{array}{l}\text { Neurological (ataxia, decreased } \\
\text { motor activity, disorientation, } \\
\text { hyperactivity, decreased sight, } \\
\text { increased sensitivity to sound), } \\
\text { Gl (vomiting, diarrhoea, } \\
\text { polyphagia), Renal } \\
\text { (increase creatinine) }\end{array}$ & $\begin{array}{l}\text { ataxia, decreased motor } \\
\text { activity, disorientation, } \\
\text { hyperactivity, decreased } \\
\text { sight, increased } \\
\text { sensitivity to sound, } \\
\text { vomiting, diarrhoea }\end{array}$ & I \\
\hline $\begin{array}{l}\text { EMA report } \\
\text { (GLP toxicity } \\
\text { study 1) }\end{array}$ & Imepitoin & 32 & $0 \%$ & $\begin{array}{l}\text { Doses of 0, } \\
31.6 \mathrm{mg} / \mathrm{kg}: 0 \% \\
\text { Other doses: } \\
\text { NA }\end{array}$ & $\begin{array}{l}\text { Doses of } 0,31.6 \\
100 \text { and } 316 \\
\mathrm{mg} / \mathrm{kg} / \text { day PO }\end{array}$ & NA & $1 \mathrm{~m}$ & $\begin{array}{l}\text { Neurological (decreased motor } \\
\text { activity), GI (hypersalivation, } \\
\text { vomiting), ECG modifications } \\
\text { No adverse effects in the } \\
\text { recommended doses; adverse } \\
\text { effects occurred only in } \\
\text { the highest doses }\end{array}$ & NA & I \\
\hline $\begin{array}{l}\text { EMA report } \\
\text { (GLP toxicity } \\
\text { study 2) }\end{array}$ & Imepitoin & NA & NA & NA & $\begin{array}{l}\text { Doses of } 0,31.6 \\
82.5 \text { and } 215 \\
\mathrm{mg} / \mathrm{kg} / \text { day PO }\end{array}$ & NA & $\begin{array}{l}3.2 \mathrm{~m} \text { (followed } \\
\text { by a } 1.2 \mathrm{~m} \\
\text { recovery period) }\end{array}$ & $\begin{array}{l}\text { Only vomiting occurred in } \\
\text { the } 0 \text { and } 31.6 \mathrm{mg} / \mathrm{kg} / \mathrm{day} \\
\text { doses; adverse effects occurred } \\
\text { only in the highest doses }\end{array}$ & NA & I \\
\hline
\end{tabular}

Abbreviations: AED(s) anti-epileptic drug(s), BID bis in die (twice daily), Chloraz chlorazepate, CSF cerebrospinal fluid, CL confidence level, Gaba Gabapentin, IE idiopathic epilepsy, LEV Levetiracetam, $m$ month(s), NA Not Available, $P H B$ phenobarbital, $P D$ polydipsia, $P U$ polyuria, $P P$ polyphagia, $P B r$, potassium bromide, Prim primidone, $P O$ per os, SID semel in die (once daily), TID ter in die (three times daily), TPM topiramate, $W$ week(s), y year(s) 
Table 3 Details of number of dogs, $95 \% \mathrm{Cl}$ affected cases, AED doses and serum levels, treatment period and adverse effects

\begin{tabular}{|c|c|c|c|c|c|c|c|c|c|c|}
\hline Studies & AED & $\begin{array}{l}\text { No of dogs } \\
\text { treated }\end{array}$ & Prevalence & $\begin{array}{l}95 \% \mathrm{Cl} \text { affected } \\
\text { cases }\end{array}$ & Doses of AEDs & $\begin{array}{l}\text { Serum levels of } \\
\text { AEDs }\end{array}$ & Treatment period & $\begin{array}{l}\text { Body system affected and } \\
\text { adverse effects }\end{array}$ & $\begin{array}{l}\text { Most common } \\
\text { adverse effects }\end{array}$ & $\begin{array}{l}\text { Adverse } \\
\text { effect type }\end{array}$ \\
\hline $\begin{array}{l}\text { Boothe et al. } \\
2012\end{array}$ & $\mathrm{PBr}$ & 23 & $78.5 \%$ & $61.7 \%-95.3 \%$ & $\begin{array}{l}\text { mean, 30.6; range, } \\
26-35 \mathrm{mg} / \mathrm{kg} \mathrm{PO} \\
\mathrm{BID}\end{array}$ & $\begin{array}{l}\text { mean, } 1.9+/-0.6 ; \\
\text { range, } 0.9-3.3 \\
\mathrm{mg} / \mathrm{ml}\end{array}$ & approximately $6 \mathrm{~m}$ & $\begin{array}{l}\text { Neurological (ataxia, } \\
\text { hyperactivity, sedation), Gl } \\
\text { (vomiting, diarrhoea, PP), } \\
\text { PU, PD }\end{array}$ & $\begin{array}{l}\text { sedation, } \\
\text { hyperactivity, } \\
\text { ataxia, PD, PU }\end{array}$ & 1 \\
\hline Pearce 1990 & $\mathrm{PBr}$ as an adjunct to $\mathrm{PHB}$ & 10 & $40 \%$ & $9.6 \%-70.4 \%$ & $\begin{array}{l}\text { PBr: } 22 \mathrm{mg} / \mathrm{kg} \\
\text { PO SID (dose } \\
\text { increases occurred) } \\
\text { PHB: median, 3.3; } \\
\text { mean, } 3.8 \mathrm{mg} / \mathrm{kg} \\
\text { PO BID (dose was } \\
\text { reduced by a mean } \\
\text { of } 50 \% \text { in } 7 / 10 \\
\text { dogs during the } \\
\text { PBr treatment) }\end{array}$ & $\begin{array}{l}\text { PBr: mean, 810; } \\
\text { range, 500-1625 } \\
\text { mg/l } \\
\text { PHB: mean, 29.7; } \\
\text { range, 17-45 } \\
\mathrm{ug} / \mathrm{ml}\end{array}$ & $\begin{array}{l}\text { median, } 7 \\
\text { mean, } 7.8 \mathrm{~m}\end{array}$ & $\begin{array}{l}\text { Neurological (ataxia, } \\
\text { sedation, hyperactivity), } \\
\text { PU, PD }\end{array}$ & $\begin{array}{l}\text { ataxia, letargy, } \\
\text { PU, PD }\end{array}$ & 1 \\
\hline $\begin{array}{l}\text { March et al. } \\
2002\end{array}$ & $\mathrm{PBr}$ & 6 & $20 \%$ & $-12.0 \%-52.0 \%$ & $\begin{array}{l}30 \mathrm{mg} / \mathrm{kg} \\
\mathrm{PO} \mathrm{BID}\end{array}$ & $\begin{array}{l}\text { median, 245; } \\
\text { range, 178-269 } \\
\mathrm{mg} / \mathrm{dL}\end{array}$ & $\begin{array}{l}3.9 \mathrm{~m} \\
\text { (adverse effects } \\
\text { occurred after this } \\
\text { period when dose } \\
\text { adjustments } \\
\text { occurred (NA)) }\end{array}$ & $\begin{array}{l}\text { Neurological (ataxia, } \\
\text { paraparesis, hyperactivity) }\end{array}$ & $\begin{array}{l}\text { ataxia, } \\
\text { paraparesis }\end{array}$ & 1 \\
\hline $\begin{array}{l}\text { Rossmeisl et al. } \\
2009\end{array}$ & $\begin{array}{l}\mathrm{PBr} \text { as an adjunct to PHB } \\
\text { and/or other AEDs }\end{array}$ & 1298 & $2 \%$ & $1.2 \%-2.8 \%$ & $\begin{array}{l}\text { PBr: } 44.9+/-1.7 \\
\mathrm{mg} / \mathrm{kg} \text { PO SID } \\
\mathrm{PHB}: 6.3+/-0.4 \\
\mathrm{mg} / \mathrm{kg} \text { PO SID }\end{array}$ & $\begin{array}{l}\mathrm{PBr}: 3.7+/-0.3 \\
\mathrm{mg} / \mathrm{ml} \\
\text { PHB: } 31.4+/-1.2 \\
\mu \mathrm{g} / \mathrm{dl}\end{array}$ & NA & $\begin{array}{l}\text { Neurological (sedation, } \\
\text { ataxia, paraparesis, } \\
\text { tetraparesis) }\end{array}$ & $\begin{array}{l}\text { sedation, } \\
\text { ataxia, } \\
\text { paraparesis, } \\
\text { tetraparesis }\end{array}$ & 1 \\
\hline $\begin{array}{l}\text { Dayrell-Hart B et } \\
\text { al. } 1996\end{array}$ & $\mathrm{PBr}$ & 238 & $10.9 \%$ & $6.9 \%-14.9 \%$ & NA & $\begin{array}{l}21 \text { affected dogs } \\
\text { had }>2.3 \mathrm{mg} / \mathrm{ml} \\
\text { and } 5 \text { affected } \\
\text { dogs had }<0.5 \\
\mathrm{mg} / \mathrm{ml}\end{array}$ & NA & $\begin{array}{l}\text { Neurological (ataxia, } \\
\text { sedation) }\end{array}$ & $\begin{array}{l}\text { ataxia, } \\
\text { sedation }\end{array}$ & 1 \\
\hline $\begin{array}{l}\text { Podell and } \\
\text { Fenner } 1993\end{array}$ & $\begin{array}{l}\mathrm{PBr} \text { as an adjunct to PHB } \\
\text { and/or other AEDs }\end{array}$ & 23 & $78 \%$ & $61.1 \%-94.9 \%$ & $\begin{array}{l}\text { PBr: mean, 20.75; } \\
\text { range, 13-40 } \\
\text { PO BID } \\
\text { PHB: NA }\end{array}$ & $\begin{array}{l}\text { PBr: } 161 \mathrm{mg} / \mathrm{dll} \\
\text { PHB: } 37.8 \mathrm{mcg} / \mathrm{ml}\end{array}$ & $\begin{array}{l}\text { mean, 15; } \\
\text { range, 4-33 m }\end{array}$ & $\begin{array}{l}\text { Neurological (ataxia, } \\
\text { sedation), GI (PP), ClinPath } \\
\text { (increased serum chloride), } \\
\text { PU, PD }\end{array}$ & $\begin{array}{l}\mathrm{PU}, \mathrm{PD}, \mathrm{PP}, \\
\text { sedation }\end{array}$ & 1 \\
\hline $\begin{array}{l}\text { Chang et al. } \\
2006\end{array}$ & $\begin{array}{l}\mathrm{PBr} \\
\text { (monotherapy } \\
\text { or as an } \\
\text { adjunct to PHB) }\end{array}$ & $\begin{array}{l}\text { Monotherapry: } 4 \\
\text { Adjunctive } \\
\text { Therapy: } 10\end{array}$ & $\begin{array}{l}\text { Monotherapry: } \\
62.5 \% \\
\text { Adjunctive } \\
\text { Therapy: } 95 \%\end{array}$ & $\begin{array}{l}\text { Monotherapy: } \\
15.0-110.0 \% \\
\text { Adjunctive therapy: } \\
81 \%-109 \%\end{array}$ & NA & NA & $\begin{array}{l}\text { median, 18; } \\
\text { range, 3-72 m }\end{array}$ & $\begin{array}{l}\text { Neurological (ataxia, } \\
\text { hyperactivity), } \\
\text { Dermatological (pruritus), } \\
\text { Gl (PP), } \\
\text { The adjunctive therapy } \\
\text { group had also PU, PD } \\
\text { and vomiting/diarhoea }\end{array}$ & $\begin{array}{l}\text { Ataxia, } \\
\text { hyperactivity, } \\
\text { pruritus, PP }\end{array}$ & 1 \\
\hline Yohn et al. 1992 & $\begin{array}{l}\mathrm{PBr} \text { as an } \\
\text { adjunct to PHB }\end{array}$ & 1 & NA & NA & NA & $2.7 \mathrm{mg} / \mathrm{ml}$ & $1 \mathrm{~m}$ & $\begin{array}{l}\text { Neurological (sedation, } \\
\text { ataxia, paraparesis, } \\
\text { anisocoria) }\end{array}$ & NA & 1 \\
\hline
\end{tabular}


Table 3 Details of number of dogs, $95 \% \mathrm{Cl}$ affected cases, AED doses and serum levels, treatment period and adverse effects (Continued)

\begin{tabular}{|c|c|c|c|c|c|c|c|c|c|c|}
\hline $\begin{array}{l}\text { Kantowitz et al. } \\
1999\end{array}$ & $\begin{array}{l}\mathrm{PBr} \\
\text { (monotherapy } \\
\text { or as an } \\
\text { adjunct to PHB) }\end{array}$ & $\begin{array}{l}\text { Monotherapry: } 15 \\
\text { Adjunctive } \\
\text { therapy: } 8\end{array}$ & NA & NA & NA & $\begin{array}{l}\text { Monotherapy: } \\
\text { median, 1985; } \\
\text { range, 500-3419 } \\
\text { mg/dL } \\
\text { Adjunctive } \\
\text { therapy: PBr: } \\
\text { median, 1399; } \\
\text { range, 584-2438 } \\
\text { mg/dL. PHB: } \\
\text { median, 22.4; } \\
\text { range, 10.9-40 } \\
\mu \mathrm{g} / \mathrm{ml}\end{array}$ & $\begin{array}{l}\text { Monotherapy: median, } \\
\text { 14.5; range, 3-37 m } \\
\text { Adjunctive therapy: } \\
\text { PBr: median, 5; range, } \\
\text { 3-72 m. PHB: } \\
\text { median, 22; range, } \\
\text { 3-96 m }\end{array}$ & $\begin{array}{l}\text { Monotherapy: Normal } \\
\text { Adjunctive therapy: } \\
\text { Endocrine (decreased } \\
\text { total T4, free T4) }\end{array}$ & NA & 1 \\
\hline $\begin{array}{l}\text { Srivastava et al. } \\
2013\end{array}$ & $\begin{array}{l}\mathrm{PBr} \text { as an } \\
\text { adjunct to PHB }\end{array}$ & 6 & $100 \%$ & $100 \%$ & $\begin{array}{l}\text { PBr: } 30 \mathrm{mg} / \mathrm{kg} \\
\text { PO SID } \\
\text { PHB: Initially } \\
2.5 \mathrm{mg} / \mathrm{kg} \text {, then } \\
5 \mathrm{mg} / \mathrm{kg} \text { PO SID. }\end{array}$ & NA & $\begin{array}{l}\text { mean, } 11.50+/-1.23 \text {; } \\
\text { range, } 8-15 \mathrm{~m} \\
\text { (on PHB). } \\
\text { Then, PBr started and } \\
3 \mathrm{~m} \text { later a reduction } \\
\text { of } 50 \% \text { in the dose of } \\
\text { PHB was performed. } \\
\text { After } 6 \mathrm{~m} \text {, PHB was } \\
\text { completely withdrawn. }\end{array}$ & $\begin{array}{l}\text { Neurological (ataxia), Gl } \\
\text { (hepatoxicity, anorexia, PP), } \\
\text { ClinPath (increased ALT, } \\
\text { ALP, AST, bile acids), } \\
\text { PU, PD } \\
\text { (polyphagia, PU, PD } \\
\text { appeared after 1-1.5 } \\
\text { years of PBr therapy) }\end{array}$ & PU, PD, PP & 1 \\
\hline Shaw et al. 1996 & $\begin{array}{l}\mathrm{PBr} \text { as an } \\
\text { adjunct to } \mathrm{PHB}\end{array}$ & 1 & NA & NA & $\begin{array}{l}\text { PBr: } 20 \mathrm{mg} / \mathrm{kg} \\
\text { PO SID } \\
\text { PHB: } 3.75 \mathrm{mg} / \mathrm{kg} \\
\text { PO BID }\end{array}$ & $\begin{array}{l}\text { PBr: } 1100 \text { mg/l. } \\
\text { PHB: } 20.4 \text { mg/ml }\end{array}$ & Approximately $21 \mathrm{~m}$ & $\begin{array}{l}\text { After PBr initiation: } \\
\text { Neurological } \\
\text { (sedation, ataxia) }\end{array}$ & NA & 1 \\
\hline Paull et al. 2003 & $\mathrm{PBr}$ & 5 & $60 \%$ & $17.1 \%-102.9 \%$ & $\begin{array}{l}\text { Initially } 100 \mathrm{mg} / \mathrm{kg} \\
\text { PO BID for } 2 \text { days. } \\
\text { Then, } \\
30 \mathrm{mg} / \mathrm{kg} \text { PO SID } \\
\text { for } 180 \text { days. }\end{array}$ & $\begin{array}{l}\text { range, } 88-300 \\
\mathrm{mg} / \mathrm{dL} \text { (only one } \\
\mathrm{dog} \text { was }>300 \\
\mathrm{mg} / \mathrm{dl} \text { ) }\end{array}$ & $6 \mathrm{~m}$ & $\begin{array}{l}\text { Endocrine (Euthyroid sick } \\
\text { syndrome with decreased } \\
\text { T4 and normal TSH) } \\
\text { Placebo group had the } \\
\text { same results }\end{array}$ & NA & 1 \\
\hline $\begin{array}{l}\text { Stabile et al. } \\
2014\end{array}$ & $\begin{array}{l}\mathrm{PBr} \text { as an } \\
\text { adjunct to PHB }\end{array}$ & 1 & NA & NA & $\begin{array}{l}\text { PBr: Initially, } \\
400 \mathrm{mg} / \mathrm{kg} \text { divided } \\
\text { in six daily doses } \\
\text { for four days. Then, } \\
14 \mathrm{mg} / \mathrm{kg} \text { PO BID } \\
\text { PHB: Initially, } \\
2.7 \mathrm{mg} / \mathrm{kg} \text {, then } \\
5 \mathrm{mg} / \mathrm{kg} \text { and finally } \\
6.4 \mathrm{mg} / \mathrm{kg} \text { PO BID. }\end{array}$ & $\begin{array}{l}\text { PBr: } 15.9 \mathrm{mg} / \mathrm{ml} ; \\
\text { PHB: } 23.7 \mu \mathrm{g} / \mathrm{ml}\end{array}$ & $\geq 26 \mathrm{~m}$ & $\begin{array}{l}\text { Neurological (sedation, } \\
\text { ataxia, generalised } \\
\text { appendicular repetitive } \\
\text { myoclonus), ClinPath } \\
\text { (pseudohyperchlormia, } \\
\text { increased ALP) }\end{array}$ & NA & $|\&| \mid$ \\
\hline $\begin{array}{l}\text { Gaskill and } \\
\text { Kimber } 2010\end{array}$ & $\mathrm{PBr}$ & 32 & $85.9 \%$ & $73.8 \%-98.0 \%$ & NA & NA & $12 \mathrm{~m}$ & $\begin{array}{l}\text { Neurological (ataxia, } \\
\text { sedation, hyperactivity, } \\
\text { aggression), Gl (PP, } \\
\text { anorexia, vomiting, } \\
\text { diarrhoea, pancreatitis), } \\
\text { Dermatological (skin } \\
\text { problems), ClinPath } \\
\text { (increased ALP, ALT, } \\
\text { amylase, lipase), PU, PD }\end{array}$ & $\begin{array}{l}\text { vomiting, } \\
\text { sedation, PP, } \\
\text { PU, PD }\end{array}$ & $|\&| \mid$ \\
\hline
\end{tabular}


Table 3 Details of number of dogs, $95 \% \mathrm{Cl}$ affected cases, AED doses and serum levels, treatment period and adverse effects (Continued)

\begin{tabular}{|c|c|c|c|c|c|c|c|c|c|c|}
\hline Volk et al. 2008 & $\begin{array}{l}\text { PBr as an } \\
\text { adjunct to PHB } \\
\text { (prior to } \\
\text { addition of } \\
\text { other AEDs) }\end{array}$ & 14 & $100 \%$ & $100 \%$ & $\begin{array}{l}\mathrm{PBr} \text { and } \mathrm{PHB}: \mathrm{NA} \\
\text { but were within } \\
\text { normal reference } \\
\text { values }\end{array}$ & $\begin{array}{l}\mathrm{PBr}: 1.7+/-0.4 \\
\mathrm{mg} / \mathrm{ml} \\
\mathrm{PHB}: 35.5+/-6.3 \\
\mu \mathrm{g} / \mathrm{ml}\end{array}$ & $\geq 2-6 \mathrm{~m}$ & $\begin{array}{l}\text { Neurological (ataxia, } \\
\text { aggression), GI (PP, } \\
\text { vomiting, pancreatitis), } \\
\text { ClinPath (increased ALT, } \\
\text { ALP) PU, PD }\end{array}$ & $\begin{array}{l}\text { increased ALT, } \\
\text { ALP, ataxia, } \\
\text { aggression }\end{array}$ & $|\&| \mid$ \\
\hline $\begin{array}{l}\text { Gaskill et al. } \\
2000\end{array}$ & $\begin{array}{l}\mathrm{PBr} \text { as an } \\
\text { adjunct to } \mathrm{PHB}\end{array}$ & $\begin{array}{l}\text { Clinical trial: } 6 \\
\text { Case series: } 19\end{array}$ & $\begin{array}{l}\text { Clinical trial: } \\
50 \% \\
\text { Case series: } \\
37 \%\end{array}$ & $\begin{array}{l}\text { Clinical trial: } \\
10.0 \%-90.0 \% \\
\text { Case series: } \\
15.3 \%-58.7 \% \\
\text { (pancreatitis); } \\
47.5 \%-89.3 \% \\
\text { (increased } \\
\text { enzymes only) }\end{array}$ & $\begin{array}{l}\text { Clinical trial: NA } \\
\text { Case series: NA }\end{array}$ & $\begin{array}{l}\text { Clinical trial: NA } \\
\text { Case series: PBr: } \\
\text { range, } 12.5-37.5 \\
\text { mmol/L; PHB: } \\
\text { range, 54-190 } \\
\text { imol/L }\end{array}$ & $\begin{array}{l}\text { Clinical trial: } \\
\text { approximately } 1 \text { year } \\
\text { Case series: NA }\end{array}$ & $\begin{array}{l}\text { Gl (pancreatitis, increased } \\
\text { amylase and/or lipase } \\
\text { activities) }\end{array}$ & $\begin{array}{l}\text { pancreatitis, } \\
\text { increased } \\
\text { amylase and/ } \\
\text { or lipase } \\
\text { activities }\end{array}$ & $\|$ \\
\hline $\begin{array}{l}\text { Steinmetz et al. } \\
2012\end{array}$ & $\begin{array}{l}\text { PBr as an } \\
\text { adjunct to PHB }\end{array}$ & 1 & NA & NA & $\begin{array}{l}101.19 \mathrm{mg} / \mathrm{kg} \\
\text { SID PO (added at } \\
\text { the beginning of } \\
\text { the } 4 \text { th year) } \\
\text { PHB: } 4.9 \mathrm{mg} / \mathrm{kg} \\
\text { BID PO }\end{array}$ & $\begin{array}{l}\text { PBr: } 45 \mathrm{mmol} / \mathrm{l} \\
\text { PHB: } \\
168.52 \mu \mathrm{mol} / \mathrm{l}\end{array}$ & $\begin{array}{l}48 \mathrm{~m} \text { (adverse effect } \\
\text { occurred after the } \\
48 \mathrm{~m} \text { ) }\end{array}$ & $\begin{array}{l}\text { Neurological } \\
\text { (neuromyopathy with } \\
\text { generalised low } \\
\text { motor signs) }\end{array}$ & NA & $\|$ \\
\hline $\begin{array}{l}\text { Mackay and } \\
\text { Mitchell } 1998\end{array}$ & $\begin{array}{l}\mathrm{PBr} \text { as an } \\
\text { adjunct to PHB }\end{array}$ & 1 & NA & NA & $\begin{array}{l}\text { PBr: Initially } \\
200 \mathrm{mg} / \mathrm{kg} \text { PO BID } \\
\text { for } 3 \text { days, then } \\
30 \mathrm{mg} / \mathrm{kg} \text { PO SID } \\
\text { PHB: } 5 \mathrm{mg} / \mathrm{kg} \\
\text { PO BID. }\end{array}$ & $\begin{array}{l}\text { PBr: NA. } \\
\text { PHB: } 126 \text { umol/L }\end{array}$ & $\begin{array}{l}3 \mathrm{~d} \\
\text { (signs started } 3 \mathrm{~d} \text { after } \\
\text { the loading dose of } \\
\mathrm{PBr} \text { was initiated) }\end{array}$ & $\begin{array}{l}\text { ClinPath (artifactual } \\
\text { hyperchloraemia and } \\
\text { negative anion gap), } \\
\text { Neurological (pacing, } \\
\text { disorientation), Gl } \\
\text { (vomiting) } \\
\text { The Neurological and Gl } \\
\text { signs were attributed to } \\
\text { hypercloraemia }\end{array}$ & NA & $\|$ \\
\hline $\begin{array}{l}\text { Boynosky and } \\
\text { Stokking } 2014\end{array}$ & $\begin{array}{l}\mathrm{PBr} \text { as an } \\
\text { adjunct to PHB }\end{array}$ & 2 & NA & NA & $\begin{array}{l}\text { Initially } 40 \mathrm{mg} / \mathrm{kg} \\
\text { PO SID, then } \\
60 \mathrm{mg} / \mathrm{kg} \text { PO SID } \\
\text { (case 1) or } 86 \mathrm{mg} / \\
\mathrm{kg} \text { PO SID (case 2) }\end{array}$ & $\begin{array}{l}\text { Case 1: } 2.9 \\
\mathrm{mg} / \mathrm{mL} \text {; Case 2: } \\
\text { initially 0.8, then } \\
3 \mathrm{mg} / \mathrm{ml} \text { (after } \\
7.5 \mathrm{months} \text { of } \\
\text { treatment) }\end{array}$ & $\begin{array}{l}12 \mathrm{~m} \\
\text { [adverse effects } \\
\text { occured } 3 \text { (case 1) and } \\
8 \text { (case } 2 \text { ) } \mathrm{m} \text { after the } \\
\text { dose increase] }\end{array}$ & $\begin{array}{l}\text { Dermatological } \\
\text { (panicculitis) accompanied } \\
\text { by sedation and anorexia }\end{array}$ & NA & $\|$ \\
\hline $\begin{array}{l}\text { Steiner et al. } \\
2008\end{array}$ & $\begin{array}{l}\mathrm{PBr} \\
\text { (monotherapy } \\
\text { or as an } \\
\text { adjunct to PHB) }\end{array}$ & $\begin{array}{l}\text { Monotherapy: } 98 \\
\text { Adjunctive } \\
\text { therapy: } 121\end{array}$ & $14 \%$ & $\begin{array}{l}\text { Monotherapy: } \\
8.2 \%-22.4 \% \\
\text { Adjunctive: } \\
5.9 \%-17.3 \%\end{array}$ & NA & $\begin{array}{l}\text { range, } 0.5-4.2 \\
\mathrm{mg} / \mathrm{ml} \text { (majority } \\
\text { of dogs; range, } \\
1-2 \mathrm{mg} / \mathrm{ml} \text { ) }\end{array}$ & NA & ClinPath (increased cPLI) & NA & $\|$ \\
\hline $\begin{array}{l}\text { Bizzeti et al. } \\
2006\end{array}$ & $\begin{array}{l}\mathrm{PBr} \text { as an } \\
\text { adjunct to PHB }\end{array}$ & 7 & $43 \%$ & $6.1 \%-79.4 \%$ & NA & NA & NA & $\begin{array}{l}\text { Pancreatitis, ClinPath } \\
\text { (increased amylase, } \\
\text { lipase,CPLI) }\end{array}$ & NA & $\|$ \\
\hline
\end{tabular}

Abbreviations: AED(s) anti-epileptic drug(s), BID bis in die (twice daily), Chloraz Chlorazepate, CSF cerebrospinal fluid, CL confidence level, Gaba Gabapentin, IE idiopathic epilepsy, LEV Levetiracetam, $m$ month(s), NA Not Available, PHB phenobarbital, PD polydipsia, PU polyuria, PP polyphagia, PBr potassium bromide, Prim primidone, PO per os, SID semel in die (once daily), TID ter in die (three times daily); TPM Topiramate; $w$ week(s), y year(s) 
Table 4 Details of number of dogs, $95 \% \mathrm{Cl}$ affected cases, AED doses and serum levels, treatment period and adverse effects

\begin{tabular}{|c|c|c|c|c|c|c|c|c|c|c|}
\hline Studies & AED & $\begin{array}{l}\text { No of } \\
\text { Dogs }\end{array}$ & Prevalence & $\begin{array}{l}95 \% \mathrm{Cl} \\
\text { affected case }\end{array}$ & Doses of AEDs & Serum levels of AEDs & Treatment period & $\begin{array}{l}\text { Body system affected and } \\
\text { adverse effects }\end{array}$ & $\begin{array}{l}\text { Most common } \\
\text { adverse effects }\end{array}$ & $\begin{array}{l}\text { Adverse } \\
\text { effect type }\end{array}$ \\
\hline $\begin{array}{l}\text { Volk et al. } \\
2008\end{array}$ & $\begin{array}{l}\text { LEV as an adjunct } \\
\text { to PHB and/or PBr }\end{array}$ & 14 & $7.14 \%$ & $-6.3 \%-20.6 \%$ & $\begin{array}{l}\text { LEV: } 10 \mathrm{mg} / \mathrm{kg} \text { for } 2 \mathrm{~m}, 20 \mathrm{mg} / \mathrm{kg} \\
\text { for further } 2 \mathrm{~m}, 10-20 \mathrm{mg} / \mathrm{kg} \\
\text { until } 6 \mathrm{~m} \text { and then } 10-20 \mathrm{mg} / \mathrm{kg} \\
\text { long-term PO TID } \\
\text { PHB and PBr: NA but were within } \\
\text { normal reference values }\end{array}$ & $\begin{array}{l}\text { PHB: } 35.5+/-6.3 \mu \mathrm{g} / \mathrm{ml}, \\
\text { PBr: } 1.7+/-0.4 \mathrm{mg} / \mathrm{ml}, \\
\text { (prior LEV initiation and } \\
2 \mathrm{~m} \text { after initiation). }\end{array}$ & $\geq 2-6 \mathrm{~m}$ & Neurological (sedation) & sedation & I \\
\hline $\begin{array}{l}\text { Volk et al. } \\
2008 \\
\text { (case series) }\end{array}$ & $\begin{array}{l}\text { LEV as an adjunct } \\
\text { to PHB and/or PBr } \\
\text { and/or gaba } \\
\text { and/or TPM }\end{array}$ & 8 & $25 \%$ & $-2.6 \%-18.6 \%$ & $\begin{array}{l}\text { LEV: } 30-32 \mathrm{mg} / \mathrm{kg} \text { PO TID TID } \\
\text { PHB and PBr: NA but were } \\
\text { within normal reference values }\end{array}$ & NA & $\begin{array}{l}\text { Approximately } \\
2-3 \mathrm{~m}\end{array}$ & Neurological (sedation) & sedation & I \\
\hline $\begin{array}{l}\text { Muñana } \\
\text { et al. } 2012\end{array}$ & $\begin{array}{l}\text { LEV as an adjunct } \\
\text { to PHB and/or PBr } \\
\text { and/or gaba } \\
\text { and/or zonisamide }\end{array}$ & 28 & $57 \%$ & $38.7 \%-75.3 \%$ & $\begin{array}{l}\text { LEV: median, 20.6; range, } \\
\text { 17-23.1 PO TID } \\
\text { PHB: median, 7.2; range, } \\
3.8-17.2 \mathrm{mg} / \mathrm{kg} \text { PO SID. } \\
\text { PBr: median, 34.0; range, } \\
13.6-84.2 \mathrm{mg} / \mathrm{kg} \text { PO SID }\end{array}$ & $\begin{array}{l}\text { LEV: range, }<2-50.8 \\
\mu \mathrm{gg} / \mathrm{mL} \text {. } \\
\text { PHB: mean, 28.13; } \\
\text { range, 15.77-36.40 } \mu \mathrm{g} / \mathrm{mL} \\
\text { PBr: mean, 186.20; } \\
\text { range, } 71.18-390 \mathrm{mg} / \mathrm{dL}\end{array}$ & $\begin{array}{l}9 \mathrm{~m} \text { (during } \\
\text { the } 5 \text { th } \mathrm{m} \text { no } \\
\text { AED was } \\
\text { administered) }\end{array}$ & $\begin{array}{l}\text { Neurological (ataxia, } \\
\text { hyperactivity), Gl: } \\
\text { (anorexia, vomiting) }\end{array}$ & ataxia & । \\
\hline $\begin{array}{l}\text { Steinberg } \\
2004\end{array}$ & $\begin{array}{l}\text { LEV as an adjunct } \\
\text { to } \mathrm{PHB} \text { and } \mathrm{PBr}\end{array}$ & 15 & $0 \%$ & $0 \%$ & $\begin{array}{l}\text { LEV: range, } 7.1-23.8 \mathrm{mg} / \mathrm{kg} \\
\text { PO TID } \\
\text { PB and PBr: NA }\end{array}$ & $\begin{array}{l}\text { PHB: mean, } \\
32.1+/-14.4 \mu \mathrm{g} / \mathrm{ml} . \\
\text { LEV: NA } \\
\text { PBr: } 2.2+/-0.7 \mathrm{mg} / \mathrm{dl}\end{array}$ & $\begin{array}{l}\text { median, 38; } \\
\text { range, } \\
13.8-95.5 \mathrm{~m}\end{array}$ & $\begin{array}{l}\text { No adverse effects } \\
\text { attributed to LEV }\end{array}$ & NA & I \\
\hline $\begin{array}{l}\text { Packer } \\
\text { et al. } 2015\end{array}$ & $\begin{array}{l}\text { LEV as an adjunct } \\
\text { to } \mathrm{PHB} \text { and } \mathrm{PBr}\end{array}$ & 52 & $46 \%$ & $32.5 \%-59.6 \%$ & $\begin{array}{l}\text { Maintenance group: mean, } 19.5 ; \\
\text { range, } 9-26 \mathrm{mg} / \mathrm{kg} \text { PO TID. } \\
\text { Pulse group: Initial dose at } 60 \\
\mathrm{mg} / \mathrm{kg} \text { followed by } 20 \\
\mathrm{mg} / \mathrm{kg} \text { PO TID }\end{array}$ & NA & $\begin{array}{l}\text { Maintenance } \\
\text { group: mean, } \\
\text { 1.4; range, } \\
0.3-7.5 \mathrm{y} \\
\text { Pulse group: } \\
\text { mean, 0.8; } \\
\text { range, 0.3-3.4 y }\end{array}$ & $\begin{array}{l}\text { Neurological (ataxia, } \\
\text { sedation, aggression, } \\
\text { hyperactivity), GI (PP, } \\
\text { vomiting, diarrhoea), PD } \\
\text { (Three times more often } \\
\text { in the pulse group) }\end{array}$ & ataxia, sedation & I \\
\hline $\begin{array}{l}\text { Fredso } \\
\text { et al. } 2015\end{array}$ & LEV & 6 & $84 \%$ & $53.8 \%-113.2 \%$ & $\begin{array}{l}\text { median, 31; mean, 30.4; } \\
\text { range, 27.6-51.5 PO TID }\end{array}$ & $\begin{array}{l}\text { median, 114; mean, 93; } \\
\text { range, 18-137 } \mu \mathrm{mol} / \mathrm{L}\end{array}$ & $2-12 m$ & $\begin{array}{l}\text { Neurological (ataxia, } \\
\text { sedation, hyperactivity, } \\
\text { disobedience, attention } \\
\text { seeking), GI (PP, anorexia, } \\
\text { vomiting), PU, PD }\end{array}$ & PP & I \\
\hline $\begin{array}{l}\text { Moore } \\
\text { et al. } 2010\end{array}$ & LEV & 6 & $16.6 \%$ & $-13.2 \%-46.4 \%$ & $\begin{array}{l}\text { At day one, a single dose was } \\
\text { administered: mean, } 21.7 ; \\
\text { range, } 20.8-22.7 \mathrm{mg} / \mathrm{kg} \text { PO. } \\
\text { Then: range, } 20.8-22.7 \mathrm{mg} / \mathrm{kg} \\
\text { PO TID for } 6 \mathrm{~d}\end{array}$ & $289.31+/-51.68 \mu \mathrm{g} / \mathrm{mL}$ & $0.25 \mathrm{~m}$ & $\begin{array}{l}\text { Gl (vomiting) } \\
\text { (only one episode at } \\
\text { the first d) }\end{array}$ & NA & । \\
\hline
\end{tabular}

Abbreviaions: AED(s) anti-epileptic drug(s), BID bis in die (twice daily), Chloraz Chlorazepate, CSF cerebrospinal fluid, CL confidence level, Gaba Gabapentin, IE idiopathic epilepsy, LEV Levetiracetam, $m$ month(s), NA Not Available, PHB phenobarbital, $P D$ polydipsia, PU polyuria, PP polyphagia, $P B r$ potassium bromide, Prim primidone, $P O$ per os, SID semel in die (once daily), TID ter in die (three times daily), TPM Topiramate, $w$ week(s), y year(s) 
Table 5 Details of number of dogs, $95 \% \mathrm{Cl}$ affected cases, AED doses and serum levels, treatment period and adverse effects

\begin{tabular}{|c|c|c|c|c|c|c|c|c|c|c|}
\hline Study & AED & $\begin{array}{l}\text { No of } \\
\text { dogs }\end{array}$ & Prevalence & $\begin{array}{l}95 \% \mathrm{Cl} \\
\text { affected case }\end{array}$ & Doses of AEDs & $\begin{array}{l}\text { Serum levels } \\
\text { of AEDs }\end{array}$ & $\begin{array}{l}\text { Treatment } \\
\text { period }\end{array}$ & $\begin{array}{l}\text { Body system affected } \\
\text { and adverse effects }\end{array}$ & $\begin{array}{l}\text { Most common } \\
\text { adverse effects }\end{array}$ & $\begin{array}{l}\text { Adverse } \\
\text { effect type }\end{array}$ \\
\hline $\begin{array}{l}\text { von Klopmann } \\
\text { et al. } 2007\end{array}$ & $\begin{array}{l}\text { Zonisamide as an } \\
\text { adjunct to PHB } \\
\text { and/or PBr }\end{array}$ & 11 & $72.7 \%$ & $46.4 \%-99.0 \%$ & $\begin{array}{l}\text { Zonisamide: mean, 8.9; range, } \\
5-11 \mathrm{mg} / \mathrm{kg} \text { PO BID } \\
\text { PHB and PBr: NA but } \\
\text { continued unchanged or } \\
\text { reduced if appropriate }\end{array}$ & $\begin{array}{l}\text { Zonisamide: median, } \\
19.2 ; \text { range, } 15.2-38 . \\
4 \mathrm{lg} / \mathrm{ml} \text {. } \\
\text { PHB: median, } 121 ; \\
\text { range, } 66-1505 \mathrm{Imol} / \mathrm{l} . \\
\text { PBr: median } 1.2 ; \\
\text { range, } 0.7-1.7 \mathrm{~g} / \mathrm{l} .\end{array}$ & $\begin{array}{l}\text { range, } \\
4-17 \mathrm{~m}\end{array}$ & $\begin{array}{l}\text { Neurological (ataxia, sedation), } \\
\text { ClinPath (increased ALP, ALT } \\
\text { and GLDH) }\end{array}$ & $\begin{array}{l}\text { ataxia, sedation, } \\
\text { increased ALP }\end{array}$ & 1 \\
\hline $\begin{array}{l}\text { Chung et al. } \\
2012\end{array}$ & Zonisamide & 10 & $10 \%$ & $-8.6 \%-28.6 \%$ & $\begin{array}{l}\text { median } 9.5 ; \text { mean } 8.65 ; \\
\text { range } 2.5-12 \mathrm{mg} / \mathrm{kg} \text { PO BID }\end{array}$ & $\begin{array}{l}\text { range, } \\
15.24-22.41 \mathrm{mg} / \mathrm{mL}\end{array}$ & $\begin{array}{l}\text { median, } 12 ; \\
\text { mean, } 11.2 \mathrm{~m}\end{array}$ & $\begin{array}{l}\text { Neurological (sedation), } \\
\text { Gl (vomiting, anorexia) }\end{array}$ & $\begin{array}{l}\text { sedation, vomiting, } \\
\text { anorexia }\end{array}$ & 1 \\
\hline $\begin{array}{l}\text { Dewey et al. } \\
2004\end{array}$ & $\begin{array}{l}\text { Zonisamide as an } \\
\text { adjunct to PHB } \\
\text { and/or PBr and/or } \\
\text { felbamate and/or } \\
\text { gaba and/or cloraz }\end{array}$ & 12 & $50 \%$ & $21.7 \%-78.3 \%$ & $\begin{array}{l}\text { Zonisamide: mean, 8.9; range, } \\
5-11 \mathrm{mg} / \mathrm{kg} \text { PO BID. } \\
\text { Other AEDs: NA but in } 9 / 12 \\
\text { dogs concurrent AEDs doses } \\
\text { were eliminated or reduced. }\end{array}$ & $\begin{array}{l}\text { Zonisamide: } \\
\text { median, } 23.5 ; \\
\text { mean, } 21.2 \mu \mathrm{g} / \mathrm{mL} \text {. }\end{array}$ & $\begin{array}{l}\text { mean, 8; } \\
\text { median, } 9 ; \\
\text { range, 2-18 m }\end{array}$ & $\begin{array}{l}\text { Neurological (ataxia, sedation), } \\
\text { Gl (vomiting), ClinPath (ALP) }\end{array}$ & $\begin{array}{l}\text { ataxia, } \\
\text { increased ALP }\end{array}$ & 1 \\
\hline $\begin{array}{l}\text { Walker et al. } \\
1988\end{array}$ & Zonisamide & 40 & NA & NA & 10,30 or $75 \mathrm{mg} / \mathrm{kg}$ PO SID & range, $10-140 \mathrm{ug} / \mathrm{ml}$ & $13 \mathrm{~m}$ & $\begin{array}{l}\text { Neurological (aggression) Gl } \\
\text { (emaciation), ClinPath } \\
\text { (increased ALP, decreased } \\
\text { albumin) }\end{array}$ & $\begin{array}{l}\text { aggression, } \\
\text { increased ALP, } \\
\text { decreased albumin }\end{array}$ & I \\
\hline $\begin{array}{l}\text { Boothe et al. } \\
2008\end{array}$ & Zonisamide & 8 & $0 \%$ & $0 \%$ & $\begin{array}{l}6.9 \mathrm{mg} / \mathrm{kg} \mathrm{IV} \mathrm{SID} \mathrm{or} \\
10.3 \mathrm{mg} / \mathrm{kg} \text { PO SID }\end{array}$ & range, $6-55 \mathrm{mcg} / \mathrm{ml}$ & $2 \mathrm{~m}$ & $\begin{array}{l}\text { Endocrine (Decreased } \\
\text { total T4) } \\
\text { (However, total T4 was } \\
\text { only slightly decreased at } \\
\text { the study end) }\end{array}$ & NA & I \\
\hline $\begin{array}{l}\text { Cook et al. } \\
2011\end{array}$ & Zonisamide & 1 & NA & NA & range, $7.9-8.4 \mathrm{mg} / \mathrm{kg}$ PO BID & $38 \mu \mathrm{g} / \mathrm{mL}$ & $18 \mathrm{~m}$ & $\begin{array}{l}\text { ClinPath (mixed acid } \\
\text { base disorder) }\end{array}$ & NA & $\|$ \\
\hline $\begin{array}{l}\text { Miller et al. } \\
2011\end{array}$ & Zonisamide & 1 & NA & NA & $7.7 \mathrm{mg} / \mathrm{kg}$ PO BID & NA & $0.3 \mathrm{~m}$ & Gl (hepatoxicity) & NA & $\|$ \\
\hline
\end{tabular}

Abbreviations: $A E D(s)$ anti-epileptic drug(s), BID bis in die (twice daily), Chloraz Chlorazepate, CSF cerebrospinal fluid, CL confidence level, Gaba Gabapentin, IE idiopathic epilepsy, LEV Levetiracetam, $m$ month(s), NA Not Available, PHB phenobarbital, $P D$ polydipsia, $P U$ polyuria, PP polyphagia, PBr potassium bromide, Prim primidone, $P O$ per os, SID semel in die (once daily), TID ter in die (three times daily), TPM Topiramate, $w$ week(s), y year(s) 
Table 6 Details of number of dogs, $95 \%$ Cl affected cases, AED doses and serum levels, treatment period and adverse effects

\begin{tabular}{|c|c|c|c|c|c|c|c|c|c|c|}
\hline Studies & AED & $\begin{array}{l}\text { No of } \\
\text { dogs treated }\end{array}$ & Prevalence & $\begin{array}{l}95 \% \mathrm{Cl} \\
\text { affected cases }\end{array}$ & Doses of AEDs & $\begin{array}{l}\text { Serum levels } \\
\text { of AEDs }\end{array}$ & Treatment period & $\begin{array}{l}\text { Body system affected and } \\
\text { adverse effects }\end{array}$ & $\begin{array}{l}\text { Most common } \\
\text { adverse effects }\end{array}$ & $\begin{array}{l}\text { Adverse } \\
\text { effect type }\end{array}$ \\
\hline $\begin{array}{l}\text { Schwartz-Porsche } \\
\text { et al. } 1982\end{array}$ & Prim & 30 & NA & NA & $\begin{array}{l}\text { range, } 13-100 \\
\mathrm{mg} / \mathrm{kg} \text { PO SID }\end{array}$ & $\begin{array}{l}\text { range, } \\
6-37 \mu \mathrm{g} / \mathrm{ml}\end{array}$ & $\begin{array}{l}\text { range } \\
6-96 \mathrm{~m}\end{array}$ & $\begin{array}{l}\text { Neurological (sedation, hind limb } \\
\text { weakness), GI (PP), PU, PD, } \\
\text { ClinPath (ALP, SGPT) }\end{array}$ & sedation, PU, PD & 1 \\
\hline $\begin{array}{l}\text { Schwartz- } \\
\text { Porsche et al. } \\
1985\end{array}$ & Prim & 20 & NA & NA & $\begin{array}{l}\text { range, } \\
17-107 \mathrm{mg} / \mathrm{kg} \\
\text { PO SID }\end{array}$ & $\begin{array}{l}\text { range, } \\
0.5-58 \mu \mathrm{g} / \mathrm{ml}\end{array}$ & $\begin{array}{l}\text { mean, 14; range, 6.0- } \\
35 \mathrm{~m}\end{array}$ & $\begin{array}{l}\text { Neurological (sedation, hind limb } \\
\text { weakness, ataxia), GI (PP), PD, } \\
\text { ClinPath (ALT, ALP, GLDH) }\end{array}$ & $\begin{array}{l}\text { sedation, PU, } \\
\text { increased ALT, ALP } \\
\text { and GLDH }\end{array}$ & I \\
\hline $\begin{array}{l}\text { Farnbach et al. } \\
1984\end{array}$ & Prim & 23 & $4.3 \%$ & $-4.0 \%-12.6 \%$ & $\begin{array}{l}\text { range, } 15.2-82 \\
\mathrm{mg} / \mathrm{kg} \text { PO SID }\end{array}$ & $\begin{array}{l}\text { range, } \\
4.8-70.7 \mu \mathrm{g} / \mathrm{ml}\end{array}$ & NA & Neurological (sedation, ataxia) & sedation, ataxia & I \\
\hline $\begin{array}{l}\text { Cunningham et al. } \\
1983\end{array}$ & Prim & 15 & NA & NA & $\begin{array}{l}10.6-39.4 \\
\mathrm{mg} / \mathrm{kg} \text { PO TID }\end{array}$ & $\begin{array}{l}\text { mean, } \\
2.4 \mu \mathrm{g} / \mathrm{ml}\end{array}$ & $9 \mathrm{~m}$ & $\begin{array}{l}\text { Neurological (disorientation, ataxia, } \\
\text { hyperactivity, pacing), GI (PP), PU, PD }\end{array}$ & $\begin{array}{l}\text { PU, PD, PP, } \\
\text { drowsiness, ataxia }\end{array}$ & I \\
\hline $\begin{array}{l}\text { Poffenbarger et al. } \\
1985\end{array}$ & $\begin{array}{l}\text { Prim (monotherapy or } \\
\text { as an adjunct to PHB) }\end{array}$ & 3 & NA & NA & Varied & NA & 8-84 m & $\begin{array}{l}\text { Gl (chronic hepatopathy/ } \\
\text { toxicity-hepatic chirosis) }\end{array}$ & $\begin{array}{l}\text { chronic } \\
\text { hepatopathy/toxicity }\end{array}$ & I \\
\hline Bunch et al. 1987 & Prim & 1 & NA & NA & $13 \mathrm{mg} / \mathrm{kg}$ PO BID & NA & $4 d$ & Neurological (hyperactivity) & NA & I \\
\hline Bunch et al. 1984 & Prim & 22 & $93 \%$ & $82.3 \%-103.6 \%$ & $\begin{array}{l}\text { Prim: } 33+/-19 \\
\text { mg/kg PO SID } \\
\text { Other AEDs: NA }\end{array}$ & NA & range, $6-120 \mathrm{~m}$ & $\begin{array}{l}\text { Gl (chronic hepatopathy/toxicity), } \\
\text { ClinPath (increased ALP, ALT, AST, } \\
\gamma \text {-GT, bile acids) }\end{array}$ & $\begin{array}{l}\text { chronic hepatoxicity, } \\
\text { increased ALP, ALT, } \\
\text { AST, bile acids }\end{array}$ & I \\
\hline Bunch et al. 1982 & Prim & 2 & NA & NA & $\begin{array}{l}\text { Case 1: } 750 \mathrm{mg} \\
\text { in total PO BID } \\
\text { Case 2: } 250 \mathrm{mg} \\
\text { in total PO BID }\end{array}$ & NA & $24 \mathrm{~m}$ & GI (chronic hepatopathy/toxicity) & chronic hepatoxicity & 1 \\
\hline $\begin{array}{l}\text { EPAR (US field } \\
\text { trial) }\end{array}$ & Prim & 110 & NA & NA & NA & NA & NA & $\begin{array}{l}\text { Neurological (ataxia, hyperactivity, } \\
\text { anxiety, disorientation), ClinPath } \\
\text { (increased enzymes-unclear which) } \\
\text { tachypnoea, PD }\end{array}$ & $\begin{array}{l}\text { ataxia, PD, increased } \\
\text { liver enzymes }\end{array}$ & 1 \\
\hline $\begin{array}{l}\text { Raw and Gaskell } \\
1985\end{array}$ & Prim & 52 & $35 \%$ & $22.0 \%-48.0 \%$ & NA & NA & $48 \mathrm{~m}$ & Neurological (ataxia), GI (PP), PD & ataxia, PD, PP & \\
\hline $\begin{array}{l}\text { Meyer and } \\
\text { Noonan } 1981\end{array}$ & Prim & 6 & $100 \%$ & $100 \%$ & $\begin{array}{l}30-40 \mathrm{mg} / \mathrm{kg} \\
\text { PO BID }\end{array}$ & NA & $3 \mathrm{~m}$ & ClinPath (increased ALP, ALT) & increased ALT, ALP & I \\
\hline $\begin{array}{l}\text { Sturtevant et al. } \\
1977\end{array}$ & Prim & 2 & $100 \%$ & $100 \%$ & $\begin{array}{l}17.6 \mathrm{mg} / \mathrm{kg} \\
\text { POTID }\end{array}$ & NA & $1 \mathrm{~m}$ & ClinPath (anemia, increased ALP) & increased ALP & |\& || \\
\hline Jacobs et al. 1998 & Prim & 1 & NA & NA & $25 \mathrm{mg} / \mathrm{kg} \mathrm{PO} B \mathrm{BI}$ & NA & $2 \mathrm{~m}$ & $\begin{array}{l}\text { Blood dyscrasias (neutropenia, } \\
\text { anemia, thrombocytopenia), } \\
\text { ClinPath (decreased albumin, } \\
\text { increased ALP) }\end{array}$ & NA & $\|$ \\
\hline Henricks 1987 & Prim & 1 & NA & NA & $\begin{array}{l}62 \mathrm{mg} \text { in total } \\
\mathrm{PO} \mathrm{BID}\end{array}$ & NA & $2 m$ & Dermatitis & NA & $\|$ \\
\hline Balazs et al. 1978 & Prim & 4 & $0 \%$ & $0 \%$ & $\begin{array}{l}40-80 \mathrm{mg} / \mathrm{kg} \\
\mathrm{PO} \mathrm{SID}\end{array}$ & NA & $1.75 \mathrm{~m}$ & No adverse effects & NA & NA \\
\hline Bunch et al. 1985 & Prim & 6 & $0 \%$ & $0 \%$ & NA & NA & NA & No adverse effects & NA & NA \\
\hline
\end{tabular}

Abbreviations: AED(s) anti-epileptic drug(s), BID bis in die (twice daily), Chloraz Chlorazepate, CSF cerebrospinal fluid, CL confidence level, Gaba Gabapentin, IE idiopathic epilepsy, LEV Levetiracetam, $m$ month(s), NA Not Available, PHB phenobarbital, PD polydipsia, PU polyuria, PP polyphagia, PBr potassium bromide, Prim primidone, PO per os, SID semel in die (once daily), TID ter in die (three times daily), TPM Topiramate, $w$ week(s), y year(s) 
Table 7 Details of number of dogs, $95 \% \mathrm{Cl}$ affected cases, AED doses and serum levels, treatment period and adverse effects

\begin{tabular}{|c|c|c|c|c|c|c|c|c|c|c|}
\hline Studies & AED & No of dogs & Prevalence & $\begin{array}{l}95 \% \mathrm{Cl} \\
\text { affected cases }\end{array}$ & Doses of AEDs & $\begin{array}{l}\text { Serum levels } \\
\text { of AEDs }\end{array}$ & $\begin{array}{l}\text { Treatment } \\
\text { period }\end{array}$ & $\begin{array}{l}\text { Body system affected } \\
\text { and adverse effects }\end{array}$ & $\begin{array}{l}\text { Most common adverse } \\
\text { effects }\end{array}$ & $\begin{array}{l}\text { Adverse } \\
\text { effect type }\end{array}$ \\
\hline $\begin{array}{l}\text { Govendir et al. } \\
2005\end{array}$ & $\begin{array}{l}\text { Gaba as an } \\
\text { adjunct to } \mathrm{PHB} \\
\text { and/or } \mathrm{PBr}\end{array}$ & 17 & $76.5 \%$ & $56.3 \%-96.6 \%$ & $\begin{array}{l}\text { Gaba: median, 35; range, } 32-40 \\
\text { mg/kg PO SID. } \\
\text { PHB: median, 8; range, 6-26 } \\
\text { mg/kg PO SID. } \\
\text { PBr: median, 24; range, 14-56 } \\
\text { mg/kg PO SID. }\end{array}$ & $\begin{array}{l}\text { Gabapentin: NA } \\
\text { PHB and PBr: } \\
\text { within normal } \\
\text { reference values }\end{array}$ & $4 \mathrm{~m}$ & $\begin{array}{l}\text { Neurological (sedation, } \\
\text { ataxia), GI (PP, } \\
\text { pancreatitis, chronic } \\
\text { hepatoxicity), ClinPath } \\
\text { (increased ALP, } \\
\text { triglycerides), PU, PD }\end{array}$ & ataxia, sedation & 1 \\
\hline Platt et al. 2006 & $\begin{array}{l}\text { Gaba as an } \\
\text { adjunct to } \\
\text { PHB and } \mathrm{PBr}\end{array}$ & 11 & $54.5 \%$ & $25.1 \%-83.9 \%$ & $\begin{array}{l}\text { mean, 10.9; range, } 9.3-13.6 \\
\mathrm{mg} / \mathrm{kg} \text { PO TID }\end{array}$ & $\begin{array}{l}\text { median, 6.8; } \\
\text { mean, 8.4; } \\
\text { range, } \\
2.2-20.7 \mathrm{mg} / \mathrm{l}\end{array}$ & $3 \mathrm{~m}$ & $\begin{array}{l}\text { Neurological } \\
\text { (ataxia, sedation) }\end{array}$ & ataxia, sedation & 1 \\
\hline $\begin{array}{l}\text { Dewey } \\
\text { et al. } 2009\end{array}$ & $\begin{array}{l}\text { Pregabalin as } \\
\text { an adjunct to } \\
\text { PHB and PBr }\end{array}$ & 11 & $91 \%$ & $74.1 \%-107.9 \%$ & $\begin{array}{l}\text { Pregabalin: } 2 \mathrm{mg} / \mathrm{kg} \text { PO TID. The } \\
\text { dose was increased by } 1 \mathrm{mg} / \mathrm{kg} \\
\text { PO TID each w until } 3 \text { or } 4 \\
\mathrm{mg} / \mathrm{kg} \text { PO TID. } \\
\text { PHB and PBr: NA but were } \\
\text { within normal reference values }\end{array}$ & $\begin{array}{l}\text { Pregabalin: } \\
\text { median, } 7.3 ; \\
\text { mean, 6.4; } \\
\text { range 2-11 } \\
\mu \mathrm{g} / \mathrm{ml} \\
\text { PHB: median, } \\
27.1 ; \text { mean, 27.7; } \\
\text { range 19.8-40 } \\
\mu \mathrm{g} / \mathrm{ml} \\
\text { PBr: median, } \\
1,6 ; \text { mean, } 1.9 ; \\
\text { range, 0.2-2.81 } \\
\mathrm{mg} / \mathrm{ml}\end{array}$ & $3 \mathrm{~m}$ & $\begin{array}{l}\text { Neurological (ataxia, } \\
\text { sedation), ClinPath } \\
\text { (increased ALP, ALT) } \\
\text { PU, PD, PP were also } \\
\text { recorded but were } \\
\text { associated to the } \\
\text { combination therapy } \\
\text { with PHB and PBr }\end{array}$ & ataxia, sedation & I \\
\hline $\begin{array}{l}\text { Ruehlmann } \\
\text { et al. } 2001\end{array}$ & $\begin{array}{l}\text { Felbamate as } \\
\text { an adjunct to } \\
\text { PHB }\end{array}$ & 6 & $33.3 \%$ & $-4.4 \%-71.0 \%$ & $\begin{array}{l}\text { Felbamate: median, } 63 \text { (initial } \\
\text { dose) and } 77 \text { (final dose); range, } \\
62-220 \mathrm{mg} / \mathrm{kg} \text { PO SID. } \\
\text { PHB: } 3.75 \mathrm{mg} / \mathrm{kg} \text { PO BID } \\
\text { (discontinued } 2 \mathrm{~m} \text { after } \\
\text { felbamate initiation) }\end{array}$ & $\begin{array}{l}\text { median, } \\
\text { 35; mean, } \\
13-55 \text { mg/l }\end{array}$ & median, $9 \mathrm{~m}$ & $\begin{array}{l}\text { Haematological } \\
\text { (leucopenia, } \\
\text { lymphopenia, } \\
\text { thrombocytopenia), } \\
\text { keratoconjunctivitis } \\
\text { sicca }\end{array}$ & $\begin{array}{l}\text { leucopenia, } \\
\text { lymphopenia, } \\
\text { thrombocytopenia }\end{array}$ & 1 \\
\hline $\begin{array}{l}\text { McGee et al. } \\
1998\end{array}$ & Felbamate & NA & NA & NA & $\begin{array}{l}\text { Sub-chronic group: 250, 500, and } \\
1000 \mathrm{mg} / \mathrm{kg} \text { PO SID } \\
\text { Chronic group: } 100 \text { and } 300 \\
\text { mg/kg PO SID }\end{array}$ & $\begin{array}{l}\text { range, } \\
16.5-79 \mu \mathrm{gg} / \mathrm{ml}\end{array}$ & $\begin{array}{l}\text { Sub-chronic } \\
\text { group: } 3 \mathrm{~m} \\
\text { chronic } \\
\text { group: } 12 \mathrm{~m}\end{array}$ & $\begin{array}{l}\text { Sub-chronic group: } \\
\text { Neurological (ataxia, } \\
\text { sedation, tremors), GI } \\
\text { (vomiting, salivation), } \\
\text { ClinPath } \\
\text { (increased ALT) } \\
\text { Chronic group: } \\
\text { Neurological (ataxia, } \\
\text { limb rigidity, tremors), } \\
\text { GI (vomiting, salivation), } \\
\text { ClinPath (increased } \\
\text { ALT, ALP) }\end{array}$ & $\begin{array}{l}\text { Sub-chronic group: } \\
\text { ataxia, sedation, } \\
\text { tremors, vomiting, } \\
\text { salivation, } \\
\text { increased ALT. } \\
\text { Chronic group: } \\
\text { ataxia, limb rigidity, } \\
\text { convulsions, } \\
\text { vomiting, salivation, } \\
\text { increased ALT, ALP. }\end{array}$ & 1 \\
\hline $\begin{array}{l}\text { Dayrell- } \\
\text { Hart et al. } \\
1996\end{array}$ & $\begin{array}{l}\text { Felbamate as } \\
\text { an adjunct to } \\
\text { PHB and PBr }\end{array}$ & 16 & $25 \%$ & $3.8 \%-46.2 \%$ & NA & NA & NA & $\begin{array}{l}\text { Gl (chronic } \\
\text { hepatotoxicity) }\end{array}$ & chronic hepatotoxicity & I \\
\hline
\end{tabular}


Table 7 Details of number of dogs, $95 \% \mathrm{Cl}$ affected cases, AED doses and serum levels, treatment period and adverse effects (Continued)

\begin{tabular}{|c|c|c|c|c|c|c|c|c|c|c|}
\hline $\begin{array}{l}\text { Bunch } \\
\text { et al. } 1985\end{array}$ & $\begin{array}{l}\text { Phenytoin } \\
\text { (monotherapy } \\
\text { or as an } \\
\text { adjunct to } \\
\text { Prim) }\end{array}$ & $\begin{array}{l}\text { Monotherapy: } \\
8 \\
\text { Adjunctive } \\
\text { therapy: } 8\end{array}$ & $\begin{array}{l}\text { Monotherapy: } \\
0 \% \\
\text { Adjunctive } \\
\text { therapy: } 37 \%\end{array}$ & $\begin{array}{l}\text { Monotherapy: } \\
0 \% \\
\text { Adjunct } \\
\text { therapy: } \\
-1 \%-16.7 \%\end{array}$ & NA & NA & NA & 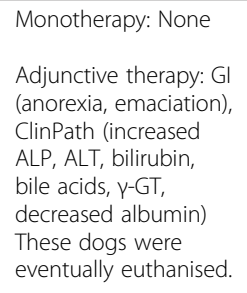 & $\begin{array}{l}\text { Monotherapy: NA } \\
\text { Adjunctive therapy: } \\
\text { anorexia, increased } \\
\text { ALP, ALT, bilirubin, } \\
\text { bile acids, } y \text {-GT, } \\
\text { decreased albumin }\end{array}$ & 1 \\
\hline $\begin{array}{l}\text { Bunch et al. } \\
1984\end{array}$ & $\begin{array}{l}\text { Phenytoin } \\
\text { (monotherapy } \\
\text { or as an } \\
\text { adjunct to } \\
\text { other AED(s)) }\end{array}$ & $\begin{array}{l}\text { Monotherapy: } \\
7 \\
\text { Adjunctive } \\
\text { therapy: } 19\end{array}$ & NA & NA & $\begin{array}{l}\text { Phenytoin: mean, } 21+/-11 \\
\text { mg/kg PO SID } \\
\text { Prim: mean, 33+/-19 mg/kg } \\
\text { PO SID. } \\
\text { Other AEDs: NA }\end{array}$ & NA & $\begin{array}{l}\text { range, } \\
6-120 \mathrm{~m}\end{array}$ & $\begin{array}{l}\text { Gl (chronic } \\
\text { hepatoxicity), ClinPath } \\
\text { (increased ALP, ALT, } \\
\text { AST, bile acids) }\end{array}$ & $\begin{array}{l}\text { Chronic } \\
\text { hepatoxicity, } \\
\text { increased ALP, ALT, } \\
\text { AST, bile acids }\end{array}$ & 1 \\
\hline $\begin{array}{l}\text { Meyer and } \\
\text { Noonan } 1981\end{array}$ & Phenytoin & 6 & $100 \%$ & $100 \%$ & 13-19 mg/kg PO TID & NA & $3 \mathrm{~m}$ & $\begin{array}{l}\text { ClinPath (increased } \\
\text { ALP, ALT) }\end{array}$ & $\begin{array}{l}\text { increased ALP and } \\
\text { ALT }\end{array}$ & 1 \\
\hline $\begin{array}{l}\text { Sturtevant } \\
\text { et al. } 1977\end{array}$ & Phenytoin & 2 & $100 \%$ & $100 \%$ & $22 \mathrm{mg} / \mathrm{kg}$ PO TID & NA & $1 \mathrm{~m}$ & $\begin{array}{l}\text { ClinPath (increased } \\
\text { ALP, ALT) }\end{array}$ & $\begin{array}{l}\text { increased ALP and } \\
\text { ALT }\end{array}$ & 1 \\
\hline $\begin{array}{l}\text { Bunch et al. } \\
1982\end{array}$ & $\begin{array}{l}\text { Phenytoin as } \\
\text { an adjunct } \\
\text { to Prim }\end{array}$ & 3 & NA & NA & $\begin{array}{l}\text { Case 1: Prim: } 250 \text { mg PO } \\
\text { BID; Phenytoin: NA. } \\
\text { Case 2: Prim: } 750 \text { mg PO BID } \\
\text { Phenytoin: } 100-233 \text { mg PO TID } \\
\text { Case 3: Prim: NA PHB: } 150 \text { mg PO } \\
\text { SID Phenytoin: } 750 \text { mg PO SID } \\
1000 \text { mg PO SID }\end{array}$ & NA & $\begin{array}{l}\text { Case 1: } \\
48 \mathrm{~m} \text {. } \\
\text { Case 2: } \\
30 \mathrm{~m} \\
\text { Case 3: } \\
36 \mathrm{~m}\end{array}$ & $\begin{array}{l}\text { Gl (chronic } \\
\text { hepatoxicity) }\end{array}$ & $\begin{array}{l}\text { chronic } \\
\text { hepatoxicity }\end{array}$ & 1 \\
\hline $\begin{array}{l}\text { Weiss et al. } \\
2002\end{array}$ & Phenytoin & 1 & NA & NA & NA & NA & NA & $\begin{array}{l}\text { Blood dyscrasias } \\
\text { (myelofibrosis) }\end{array}$ & NA & $\|$ \\
\hline $\begin{array}{l}\text { Bunch et al. } \\
1987\end{array}$ & $\begin{array}{l}\text { Phenytoin as } \\
\text { an adjunct to } \\
\text { PHB and/or } \\
\text { Prim }\end{array}$ & 3 & NA & NA & $\begin{array}{l}\text { Case 1: Phenytoin: } 5 \mathrm{mg} / \mathrm{kg} \text { PO } \\
\text { BID, then increased up to } 15 \mathrm{mg} / \\
\mathrm{kg} \text { PO TID. PHB: } 0.8 \mathrm{mg} / \mathrm{kg} \text { PO BID, } \\
\text { then increased up to } 13 \mathrm{mg} / \mathrm{kg} \text { PO } \\
\text { BID } \\
\text { Case 2: Phenytoin: } 7.5 \mathrm{mg} / \mathrm{kg} \text { PO } \\
\text { BID, then increased up to } 15 \mathrm{mg} / \\
\mathrm{kg} \text { PO TID. PHB: } 1.1 \mathrm{mg} / \mathrm{kg} \text { PO BID, } \\
\text { then increased up to } 4.5 \mathrm{mg} / \mathrm{kg} \\
\text { PO TID. Prim: } 18.5 \mathrm{mg} / \mathrm{kg} \text { PO TID } \\
\text { Case } 3 \text { : Phenytoin: } 5 \mathrm{mg} / \mathrm{kg} \text { PO } \\
\text { SID, then increased up to } 21 \mathrm{mg} / \\
\mathrm{kg} \text { PO BID. PHB: } 3 \mathrm{mg} / \mathrm{kg} \text { PO BID. } \\
\text { Prim: } 13 \mathrm{mg} / \mathrm{kg} \text { PO SID, then } \\
\text { increased up to } 26 \mathrm{mg} / \mathrm{kg} \\
\text { PO BID }\end{array}$ & $\begin{array}{l}\text { Case 1: NA } \\
\text { Case 2: NA } \\
\text { Case 3: NA }\end{array}$ & $\begin{array}{l}\text { Case 1: } \\
27 \text { months } \\
\text { Case 2: } \\
15 \text { months } \\
\text { Case 3: } \\
8 \text { months }\end{array}$ & Gl (hepatotoxicity) & NA & $\|$ \\
\hline $\begin{array}{l}\text { Nash et al. } \\
1977\end{array}$ & Phenytoin & 1 & NA & NA & $100 \mathrm{mg}$ in total & NA & $1 d$ & Idiosyncrasic hepatitis & NA & $\|$ \\
\hline $\begin{array}{l}\text { Bunch } \\
\text { et al. } 1990\end{array}$ & Phenytoin & 8 & $0 \%$ & $0 \%$ & 40 mg/kg PO TID & NA & $13.5 \mathrm{~m}$ & No adverse effects & NA & NA \\
\hline
\end{tabular}


Table 7 Details of number of dogs, $95 \%$ Cl affected cases, AED doses and serum levels, treatment period and adverse effects (Continued)

\begin{tabular}{|c|c|c|c|c|c|c|c|c|c|c|}
\hline Nafe 1981 & $\begin{array}{l}\text { Valproate } \\
\text { (monotherapy } \\
\text { or as an } \\
\text { adjunct to PHB } \\
\text { and/or Prim } \\
\text { and/or phenytoin) }\end{array}$ & $\begin{array}{l}\text { Monotherapy: } \\
\text { NA } \\
\text { Adjunctive } \\
\text { therapy: } 57\end{array}$ & $\begin{array}{l}\text { Monotherapy: } \\
\text { NA } \\
\text { Adjunctive } \\
\text { therapy: } \\
2 \%\end{array}$ & $\begin{array}{l}\text { Monotherapy: NA } \\
\text { Adjunctive } \\
\text { therapy: } \\
-1.7 \%-5.1 \%\end{array}$ & $\begin{array}{l}\text { Sodium Valproate: Monotherapy: } \\
200 \text { mg/kg. Adjunctive therapy: } \\
\text { range, 25-40 mg/kg PO SID. } \\
\text { PHB, Prim and Phenytoin: NA. }\end{array}$ & NA & $\begin{array}{l}\text { mean, } \\
4.9 ; \text { range, } \\
1-8 \mathrm{~m}\end{array}$ & $\begin{array}{l}\text { Neurological (ataxia, } \\
\text { sedation), } \\
\text { dermatological } \\
\text { (alopecia), Gl } \\
\text { (vomiting) }\end{array}$ & Sedation, alopecia & 1 \\
\hline $\begin{array}{l}\text { Kiviranta } \\
\text { et al. } 2013\end{array}$ & TPM & 10 & NA & NA & $\begin{array}{l}\text { TPM: Initially } 2 \mathrm{mg} / \mathrm{kg} \text { PO BID for } \\
0.5 \mathrm{~m} \text {, then } 5 \mathrm{mg} / \mathrm{kg} \text { PO BID for } \\
2 \mathrm{~m} \text {, and then } 10 \mathrm{mg} / \mathrm{kg} \text { PO BID } \\
\text { for } 2 \mathrm{~m} \text { and then } 10 \text { PO TID for } \\
2 \mathrm{~m} \text {. } \\
\text { PHB, PBr and LEV: NA but were } \\
\text { within normal reference values }\end{array}$ & NA & $2-6 m$ & $\begin{array}{l}\text { Neurological } \\
\text { (sedation, ataxia), } \\
\text { ClinPath } \\
\text { (increased ALP, ALT), } \\
\text { weight lose }\end{array}$ & $\begin{array}{l}\text { sedation, ataxia, increased } \\
\text { ALP, ALT }\end{array}$ & 1 \\
\hline
\end{tabular}

Abbreviations: AED(s) anti-epileptic drug(s), BID bis in die (twice daily), Chloraz Chlorazepate, CSF cerebrospinal fluid, CL confidence level, Gaba Gabapentin, IE idiopathic epilepsy, LEV Levetiracetam, $m$ month(s), NA Not Available, $P H B$ phenobarbital, $P D$ polydipsia, $P U$ polyuria, PP polyphagia, $P B r$ potassium bromide, Prim primidone, $P O$ per os, SID semel in die (once daily), TID ter in die (three times daily), TPM Topiramate, $W$ week(s), y year(s) 
but no correlation could be found between serum levels and safety profile. In one study, however [90], there was a correlation between the administered dose and the safety profile; dogs treated with a higher initial dose (i.e. $60 \mathrm{mg} / \mathrm{kg}$ ) experienced more adverse effects compared to dogs started on lower doses $(20 \mathrm{mg} / \mathrm{kg}$ ) (65 \% (95 \% CI: $43.6-86.5 \%)$ vs. $34 \%$ (95 \% CI: 14.6-53.4\%), $\mathrm{p}=0.03)$.

\section{Zonisamide}

There was an overall weak level of evidence provided for the zonisamide safety profile either as monotherapy or adjunctive therapy. Seven studies [30, 56, 57, 61, 68, 97, 99] presented data about the safety profile of oral zonisamide either as monotherapy (5 studies) [30, 61, 68, 97, 99] or as an adjunct to other AEDs (2 studies) [56, 57], giving a combined sample size of 83 dogs.

Five studies reported type I and type II adverse effects (Table 5). From these, 3/5 (60\%) and 2/5 (40\%) studies reported type I adverse effects for zonisamide monotherapy and adjunctive therapy (mainly to phenobarbital and/or potassium bromide) respectively. Specifically, in all of the studies, adverse effects most commonly included sedation, followed by ataxia, vomiting and decreased serum ALP activity. Less commonly, increased serum ALT and GLDH activity, aggression, anorexia, emaciation and decreased $\mathrm{T} 4$ and serum albumin were reported. In adjunctive therapy studies, adverse effects most commonly included sedation, ataxia and increased serum ALP activity, followed by vomiting, increased serum ALT and GLDH activity. In monotherapy studies all the adverse effects occurred at the same frequency; increased serum GLDH and ALT activity were not reported (Fig. 8). Two studies reported type II adverse effects including idiosyncratic hepatotoxicity and mixed acid-base disorder (Table 5).

Adequate data to allow calculations of the prevalence of adverse effects was reported in 4/7 (57 \%) studies (Table 5). From these, only one showed $>50 \%$ prevalence of adverse effects for the specific period of treatment they conducted. Based on the $95 \% \mathrm{CI}$, the majority of the population experienced at least one adverse effect in $25 \%$ of the studies (Table 5).

Adequate information about the treatment period was reported in all of the studies (Table 5). From these, in 3/ $6(50 \%)$ the treatment period was relatively short ( $<6$ months). Adequate information about the doses was provided in all studies (Table 5). The zonisamide maintenance doses exceeded the recommended dose margins in two studies. Adequate information about the zonisamide serum levels was reported in 6/7 (86 \%) studies (Table 5), but no correlation could be found between serum levels and the safety profile.

\section{Primidone}

There was an overall weak level of evidence provided for the primidone safety profile either as monotherapy or adjunctive therapy. Sixteen studies $[28,32,35,52,64$, $65,76,82,89,91,92,95,103-105,113$ ] presented data about the safety profile of primidone as an adjunct to phenobarbital [104] and as a monotherapy agent (remaining studies), giving a combined sample size of 298 dogs.

Twelve studies reported type I adverse effects including gastro-intestinal and neurological signs as the most common (Table 6). Specifically, adverse effects most commonly included increased serum ALP activity, ataxia and polydipsia followed by sedation, hyperactivity, chronic clinical hepatopathy/toxicity, polyphagia, disorientation and then increased serum ALT activity, hind limb weakness and polyuria. Less commonly reported effects included anxiety, tachypnea and increased serum AST, $\gamma$-GT, bile acids, SGPT and GLDH activity (Fig. 9). In two studies [32, 76] no adverse effects attributed to primidone were reported. Three studies reported type II adverse effects (Table 6). Specifically, blood dyscrasias (anemia, thrombocytopenia and/or neutropenia) and dermatitis were reported.

Adequate data to allow calculations of the prevalence of adverse effects was reported in 7/16 (44\%) of the studies (Table 6). From these, 3/7 (43\%) showed $>50 \%$ prevalence of adverse effects for the specific period of treatment over which it was conducted. Based on the $95 \% \mathrm{CI}$, the majority of the population experienced at least one adverse effect in 3/7 (43 \%) studies (Table 6).

Adequate information about the treatment period was reported in 13/16 (81 \%) (Table 6). From these, in 6/13 the treatment period was relatively short ( $<6$ months). Adequate information about the dose was provided in 14/16 (88 \%) studies (Table 6). From these 11/14 (79 \%) and 3/14 (21 \%) reported doses for type I and type II adverse effects respectively. The primidone maintenance doses were higher than the recommended dose margins in type I and type II adverse effects in 5/11 (45\%) and $1 / 3$ (33 \%) studies, respectively. Adequate information about the primidone (phenobarbital) serum levels was reported in 5/16 (31\%) studies (Table 6). Of these, all reported type I adverse effects. The phenobarbital serum levels were higher than the recommended margins in $3 / 5(60 \%)$ studies.

\section{Gabapentin}

There was an overall weak level of evidence provided for the gabapentin safety profile. Two studies $[58,59]$ presented data about the safety profile of oral gabapentin as an adjunct to other AEDs, giving a combined sample size of 28 dogs. 
Both studies reported only type I adverse effects, including neurological signs as the most common (Table 7). Specific adverse effects most commonly included sedation and ataxia. Less commonly reported adverse effects were polyphagia, pancreatitis, chronic clinical hepatopathy/toxicity, polydipsia, polyuria and increased ALP activity.

Both studies showed $>50 \%$ prevalence of adverse effects for the specific period of treatment during which they were conducted (Table 7). Based on the $95 \%$ CI, the majority of the population experienced at least one adverse effect in one study (Table 7). In both studies the treatment period was relatively short ( $<6$ months). The gabapentin maintenance doses were high but within the upper recommended dose margins in one study. Gabapentin serum levels were not measured and, thus, no analysis to detect associations between serum levels and adverse effects was performed.

\section{Pregabalin}

There was an overall insufficient level of evidence provided for the pregabalin safety profile. One study [55] presented data about the safety profile of oral pregabalin as an adjunct to phenobarbital and potassium bromide in 11 dogs.

The study reported only type I adverse effects including neurological signs as the main ones (Table 7). Specifically, sedation, ataxia and increased ALP activity and ALT activity were reported.

The study showed $91 \%$ prevalence of adverse effects for the specific treatment period in which it was conducted. Based on the $95 \% \mathrm{CI}$, the majority of the population experienced adverse effects (Table 7). The treatment period was relatively short ( $<6$ months). No correlation was found between pregabalin dose/serum levels and adverse effects.

\section{Valproate}

There was an overall weak level of evidence provided for the valproate safety profile. One study [62] presented data about the safety profile of sodium valproate in different groups either as a monotherapy or as an adjunct to phenobarbital, primidone or a combination of phenobarbital and phenytoin in 57 dogs. The study reported only type I adverse effects including neurological, gastro-intestinal and dermatological ones (Table 7). Specifically, vomiting, sedation, ataxia and alopecia were reported.

The study did not show $>50 \%$ prevalence of adverse effects for the specific period of treatment during which it was conducted. Based on the $95 \% \mathrm{CI}$, the majority of the population did not experience adverse effects (Table 7). The treatment period was unclear. No correlation was found between valproate dose and adverse effects. The serum levels were not measured.

\section{Felbamate}

There was an overall weak level of evidence provided for the felbamate safety profile either as monotherapy or adjunctive therapy. Three studies $[47,70,78]$ presented data about the safety profile of felbamate as monotherapy [47] or as an adjunct to phenobarbital in particular and potassium bromide (remaining studies), giving a combined sample size of 22 dogs.

All studies reported type I adverse effects including gastro-intestinal and neurological signs as the most common (Table 7). Specifically, adverse effects most commonly included ataxia, tremors, vomiting, hypersalivation and increased ALP activity. Less commonly, clinical hepatopathy/toxicity, increased ALT activity, sedation, limb rigidity, leucopenia, lymphopenia, thrombocytopenia and keratoconjunctivitis sicca were reported (Fig. 10).

Two studies reported type II adverse effects (Table 7). Specifically, blood dyscrasias (anemia, thrombocytopenia and neutropenia) and dermatitis were reported.

Adequate data to allow calculations of the prevalence of adverse effects was reported in $2 / 3(67 \%)$ of the studies (Table 7) and none showed $>50 \%$ prevalence of adverse effects for the specific period of treatment during which they were conducted. Based on the $95 \%$ $\mathrm{CI}$, the majority of the population did not experience adverse effects (Table 7).

Adequate information about the treatment period was reported in 2/3 (67\%) (Table 7) and it was relatively short in one study ( $<6$ months). Adequate information about the dose and serum levels was provided in $2 / 3$ (67\%) studies (Table 7). In both of these the maintenance doses were higher than the recommended dose margins. No correlation was found between serum levels and adverse effects.

\section{Topiramate}

There was an overall weak level of evidence provided for the topiramate safety profile. One study [54] presented data about the safety profile of topiramate as an adjunct to phenobarbital, potassium bromide and levetiracetam in 10 dogs. The study reported type I adverse effects, including neurological and gastrointestinal as the most common. Specific adverse effects most commonly reported were ataxia, sedation, weight loss and increased ALP and ALT activity. However, these adverse effects could be attributed in part to the co-administered AEDs such as phenobarbital and/or potassium bromide. The prevalence of adverse effects was approximately $50 \%$. The treatment period was relatively short ( $<6$ months). The maintenance dose was within recommended dose margins (Table 7). Topiramate serum levels were not measured. 


\section{Phenytoin}

There was an overall weak level of evidence provided for the phenytoin safety profile either as monotherapy or adjunctive therapy. Nine studies [32, 35, 52, 87, 89, 91, $92,108]$ presented data about the safety profile of phenytoin as monotherapy (7 studies) [32, 34, 35, 52, 87, $91,108]$ as an adjunct to primidone in particular and/or potassium bromide (4 studies) [32, 89, 91, 92], giving a combined sample of 66 dogs.

Five studies reported type I adverse effects including gastro-intestinal as the most common (Table 7). Specifically, increased serum ALP, ALT, bile acids and chronic clinical hepatopathy/toxicity were most commonly included. Less commonly, increased serum AST, $\gamma$-GT, bilirubin and bile acids activity, decreased albumin, anorexia and emaciation were reported (Fig. 11). In the monotherapy group, no adverse effects occurred in two studies [32, 34].

Three studies reported type II adverse effects (Table 7). Specifically, idiosyncratic hepatotoxicity in particular and blood dyscrasias (anemia, thrombocytopenia and/or neutropenia) occurred. Blood dyscrasias (pancytopenia) were reported by the monotherapy study [87].

Adequate data to allow calculations of the prevalence of adverse effects was reported in 4/9 (44\%) of the studies and all but two did not show $>50 \%$ prevalence of adverse effects for the specific period of treatment they were conducted. Based on the $95 \% \mathrm{CI}$, the majority of the population experienced adverse effect in two studies (Table 7).

Adequate information about the treatment period was reported in 8/9 (89 \%) (Table 7). From these, in 6/8 $(75 \%)$ the treatment period was relatively short ( $<6$ months). Adequate information about the dose and/ or serum levels was provided in 7/9 (77 \%) (Table 7). No correlation could be found between dose and adverse effects. Phenytoin serum levels were not measured.

\section{B) Safety profile comparisons between AEDs or between AEDs-placebo}

\section{Phenobarbital monotherapy vs placebo or untreated control}

Total safety information was available in five studies $[33,36,40,43,80]$. Adequate information to calculate OR was provided in all the studies. Studies compared endocrinal factors and liver enzymes between phenobarbital and untreated or placebo-control groups. Four adverse effects were reported. The common estimated OR was 6.21 (95 \% CI: 3.28-11.75), showing a statistically significant association $(\mathrm{P}<0.001)$, with reduced odds of overall adverse effects in the untreated controls. Thus, treated dogs were over 6 times as likely to experience an adverse effect compared to controls. However, moderate heterogeneity was shown between studies $\left(\mathrm{chi}^{2}=6.62\right.$, $\mathrm{P}=0.09$ ). The $\mathrm{OR}$ for abnormal total T4, TSH and increased ALP and ALT activity showed a statistically significant association, with reduced odds of these adverse effects in the controls (Fig. 12).

\section{Phenobarbital monotherapy vs potassium bromide monotherapy}

Total safety information was available in five studies [3, $23,29,39,75]$. Adequate information to calculate OR was provided in all the studies. Twelve adverse effects were reported. The common estimated OR was 0.80 (95 \% CI: 0.31-2.04), showing a statistically nonsignificant association $(P=0.64)$ between monotherapies. Low heterogeneity was shown between studies $\left(\mathrm{chi}^{2}=\right.$ $1.07, \mathrm{P}=0.59$ ). The OR for increased serum ALP activity showed a statistical trend in favour of potassium bromide. The OR for pancreatitis, vomiting and increased serum amylase and lipase activity showed a statistically significant association, with reduced odds of these adverse effects in the phenobarbital group (Fig. 13).

\section{Phenobarbital monotherapy vs levetiracetam monotherapy}

Total safety information was available in one study [27] Eleven main adverse effects were reported. The common estimated OR was 2.5 (95\% CI: 0.16-38.6), showing a statistically non-significant association $(\mathrm{P}=0.51)$ between monotherapies. The OR for hypoactivity showed a statistically significant association, with reduced odds of this adverse effect in levetiracetam monotherapy (Fig. 14). Although the number of affected dogs did not significantly differ between the groups, the frequency of adverse effects (per dog) was higher in the phenobarbital group in this study.

\section{Phenobarbital monotherapy vs imepitoin monotherapy}

Total safety information was available in two studies $[25,42]$. Adequate information to estimate OR was provided in one study [25] (Fig. 15). Four adverse effects were reported. The common estimated OR was 0.65 (95\% CI: $0.38-1.10)$, showing a statistically non-significant association $(\mathrm{P}=0.11)$ between monotherapies. The $\mathrm{OR}$ for polydipsia and polyuria showed a statistical trend in favor of imepitoin. The OR for sedation and hyperactivity showed a statistically significant association, with reduced odds of these adverse effects in imepitoin and phenobarbital, respectively.

The same study [25] reported that liver enzymes (serum ALP, $\gamma$-GT, ALT and GLDH) were increased significantly $(\mathrm{P}<0.001)$ in the phenobarbital group, along with a statistically significant $(\mathrm{P}<0.05)$ association for dose dependence; neither such increase nor dose dependence trend was seen in the imepitoin group. 


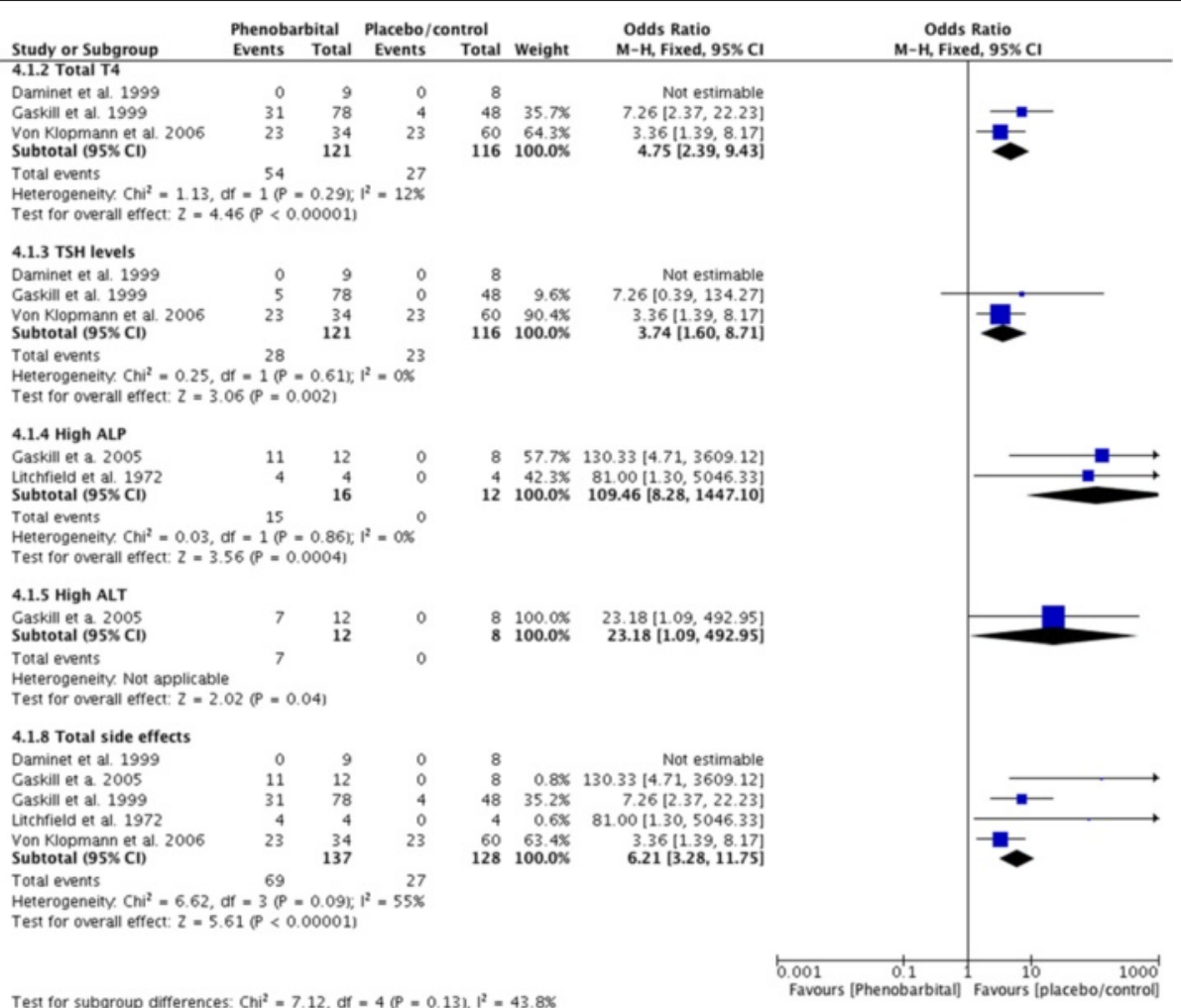

Fig. 12 Forest plot comparing phenobarbital vs placebo/control. Odd ratios ( $95 \% \mathrm{Cl}$ ) of specific and total adverse effects for phenobarbital and control groups

The second study [42], although the OR could not be estimated, reported sedation, ataxia, polydipsia and polyuria in phenobarbital group, whereas no adverse effects were observed in the imepitoin group.

\section{Imepitoin monotherapy vs imepitoin adjunctive therapy}

Total safety information was available in two studies $[44,48]$. Imepitoin monotherapy was compared to imepitoin adjunctive therapy to phenobarbital and/or potassium bromide. Three adverse effects were reported. The common estimated OR was 0.58 (95 \% CI: 0.191.75), showing a statistically non-significant association $(\mathrm{P}=0.34)$ between the two therapies. No heterogeneity was shown between studies $\left(\mathrm{chi}^{2}=0.00, \mathrm{P}=1.00\right)$. The OR for increased serum ALP activity showed a statistically significant association, with reduced odds of this adverse effect in imepitoin monotherapy (Fig. 16).

\section{Imepitoin monotherapy vs pseudo-placebo}

Total safety information was available in one study [26]. Imepitoin monotherapy (high dose) was compared to imepitoin monotherapy (low dose; pseudo-placebo group). The study grouped the adverse effects into broad categories (e.g. neurological, gastro-intestinal, etc.). The adverse effects were reported on the grounds of these categories but they were not specified. The common estimated OR was 1.39 (95 \% CI: 0.53-3.64), showing a statistically non-significant association $(\mathrm{P}=0.5)$ between monotherapy and pseudo-placebo. The OR for neurological signs (ataxia and hyperactivity/restlessness) showed a statistically significant association, with reduced odds of this adverse effect in low-dose imepitoin monotherapy (Fig. 17).

The study reported that the findings from the hematology and biochemistry evaluation were unremarkable and remained within normal reference ranges. There was only a very low tendency for serum creatinine activity to increase in the high-dose imepitoin monotherapy compared to low-dose, but this change was also within normal ranges.

\section{Levetiracetam adjunctive therapy vs placebo}

Total safety information was available in one study [24]. The study compared adverse effects between levetiracetam (as an adjunct to phenobarbital, potassium bromide, gabapentin and/or zonisamide) and placebo-control (as an adjunct to phenobarbital, potassium bromide, gabapentin and/or zonisamide). Four adverse effects were reported. The common estimated OR was 1.82 (95 \% CI: $0.62-5.35$ ), showing a statistically non-significant association $(\mathrm{P}=0.28)$ between treatment and placebo (Fig. 18). However, according to the study [24], a significant 


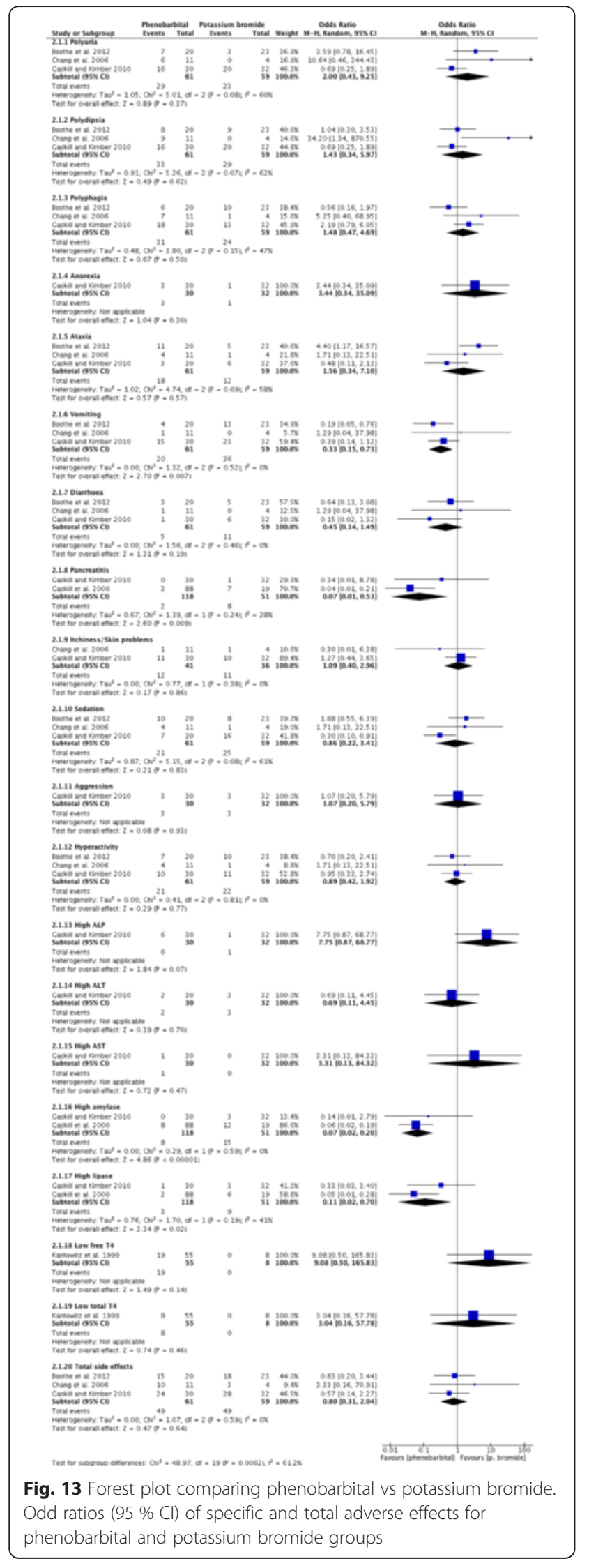

increase in the prevalence of any of the reported adverse effects $(P=0.013)$ and ataxia in particular $(\mathrm{p}=0.002)$ was noted in dogs receiving levetiracetam compared to baseline.

Further comparisons between AEDs were not possible due to the lack of comparison studies and/or insufficient report of power analysis and data to allow additional statistical analysis.

\section{Discussion}

To the authors' knowledge, this is the first systematic review, including a meta-analytic approach, of AEDs' adverse effects in dogs. The authors followed the PRISMA statement to report this systematic review [120]. The potential for development of AEDs' adverse effects in dogs are well accepted. Our systematic review found, however, that the strength of evidence as well as the prevalence and occurrence of adverse effects were variable among different AEDs and even among studies evaluating the same AED.

Main categories of adverse effects are type I (pharmacology-related) and type II (idiosyncratic). While type I effects are dose-dependent, occur predictably and are usually caused by a known pharmacological property of the drug, idiosyncratic reactions cannot be explained on the grounds of the drug's known mechanisms of action and usually occur unpredictably and irrespective of dosage [121]. In our study, type II adverse effects occurred mainly in dogs receiving doses within the recommended margins; however, these adverse effects occurred at any dose. Type II adverse effects occurred in primidone, phenobarbital, potassium bromide and zonisamide. Idiosyncratic reactions were usually caused either by immune-mediated hypersensitivity reactions or by cytotoxic effects of the drug or one of its metabolites [121]. Due to the liver's central role in drug metabolism it is one of the major sites where idiosyncratic drug reactions manifest. Individual differences in rate of formation and detoxification of reactive metabolites may explain why only certain patients develop idiosyncratic reactions [121]. These unpredictable adverse reactions occur rarely and therefore frequently remain undetected during clinical trials until approval and marketing of drugs. Once a large number of patients is exposed to new or more AEDs, these adverse effects may increase in frequency [122].

This systematic review identified and evaluated ninety studies, the vast majority of which were UCTs, retrospective case series, and reports derived from second and third groups. In total, the data of 4102 dogs were included. Direct and indirect comparisons among drugs based on the frequency, proportion and prevalence of adverse effects in each AED and study showed that levetiracetam and secondary imepitoin might be amongst 


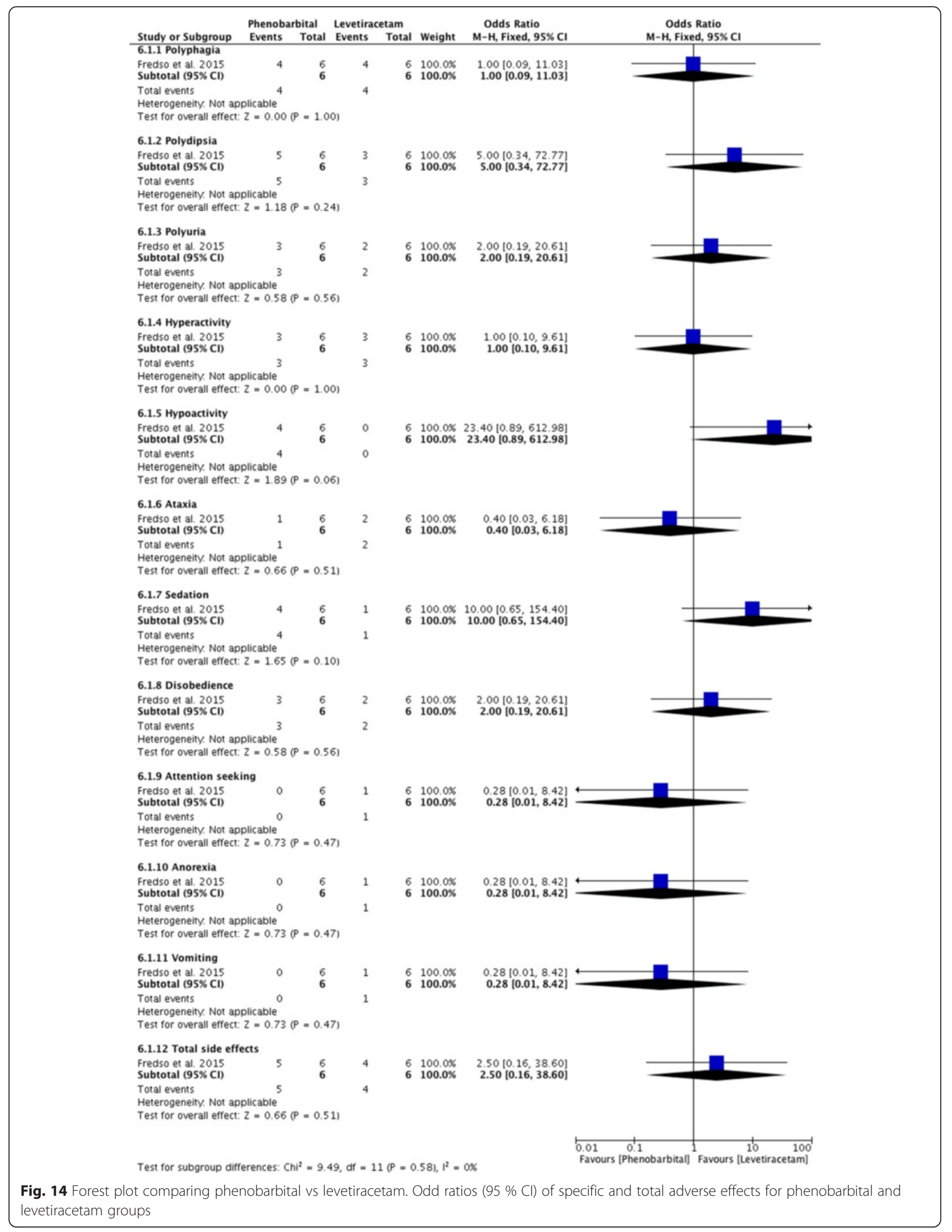




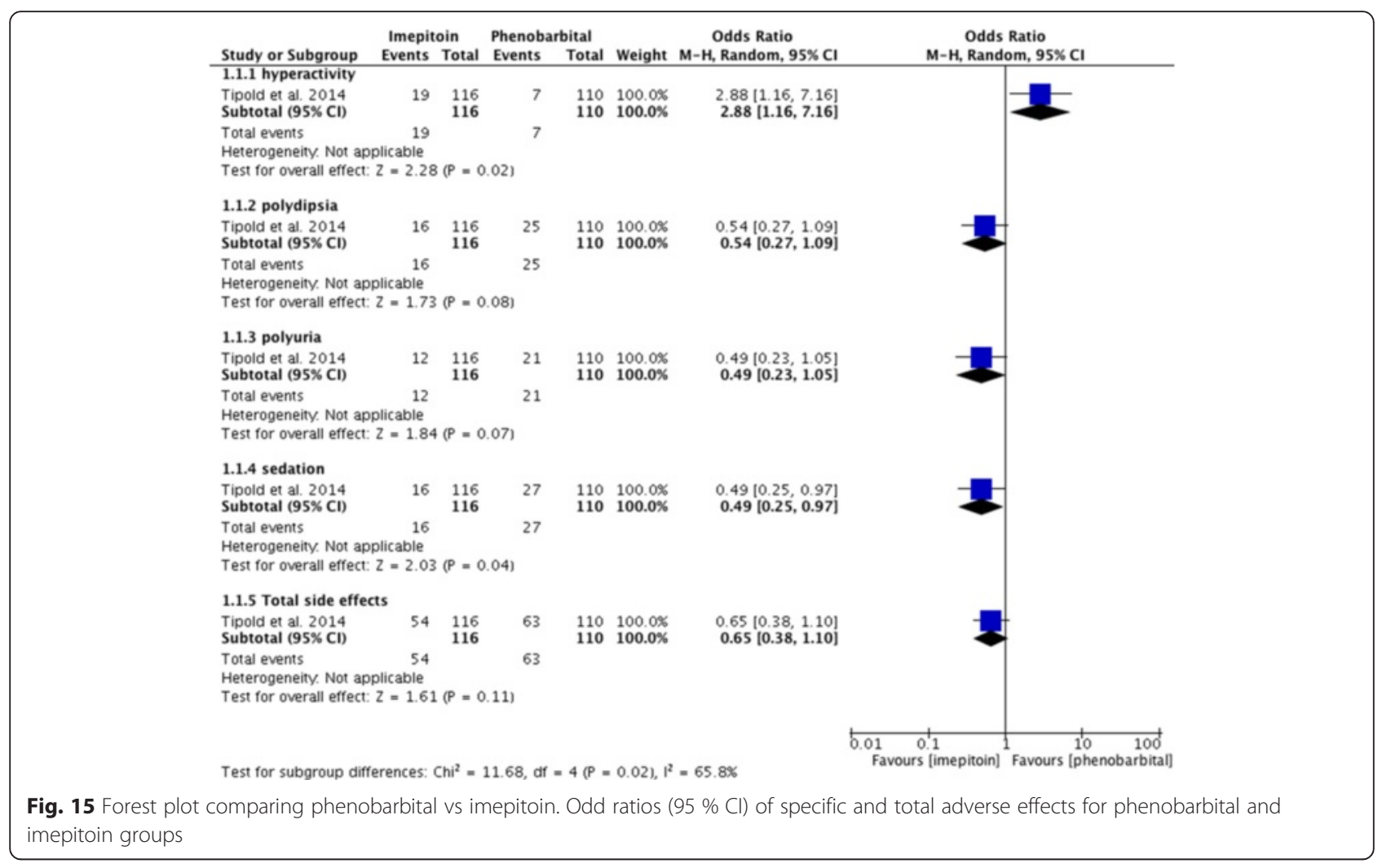

the safest AEDs, followed by phenobarbital and potassium bromide; a strong level of evidence was identified supporting their safety profile. The remaining AEDs showed a variety of adverse effects, but there was weak evidence to support their safety profile. Direct comparisons could be made only between specific AEDs based on the results of comparison group studies. It was found that imepitoin tended to have a better safety profile than phenobarbital. Levetiracetam appeared to have a better safety profile than phenobarbital, and phenobarbital appeared to have a better safety profile than potassium bromide. However, neither association was statistically significant. The trend for phenobarbital to have a safer profile than potassium bromide supports a former highquality study which showed that phenobarbital had a better safety profile than potassium bromide [23]. It is interesting that this association was not statistically different in our meta-analysis. It could be that our statistical methods applied were too stringent or that data in our study were more heterogenous, due to the data deriving from various studies, making it less likely in this systematic review to detect a statistical difference. Equally though, it is acknowledged there may be no major underlying biological difference in adverse event rates between these therapies.

Similar to a previous systematic review of AED efficacy [13], the majority of the studies included in this review did not offer high quality of evidence. Studies in the first group, which were considered to offer lower overall risk of bias, were too few compared to other groups (study group 1st:2nd:3rd proportion was 1:4:3). In addition, only 16 and $33 \%$ of studies included well-characterised groups of IE and healthy non-epileptic dogs respectively. Only $13 \%$ of studies included good study population size. Many studies had only a very limited follow-up time to assess efficacy and tolerability ( $<6$ months). Therefore, the results should always be interpreted with caution.

In contrast to AEDs' efficacy evaluation, case reports do have an important role in forming a safety profile of an individual AED. Specifically, type II or other rare adverse events are unlikely to be recognized by standard clinical trials [123]. Searching the whole range of publications and including NRCTs, UCTs, observational and descriptive studies might avoid missing important information about rare adverse effects and removes potential biases that could occur in systematic reviews which use stricter exclusion criteria and do not include studies other than RCTs [124]. A number of reviews of human drugs have found that case reports and spontaneous reporting systems provide better information about adverse effects than clinical trials do [115, 125]. Indeed, in our review, we found that case reports provided valuable specifics for several kinds of adverse effects not reported in other study designs. Therefore, although small case series and in particular case reports are considered to 


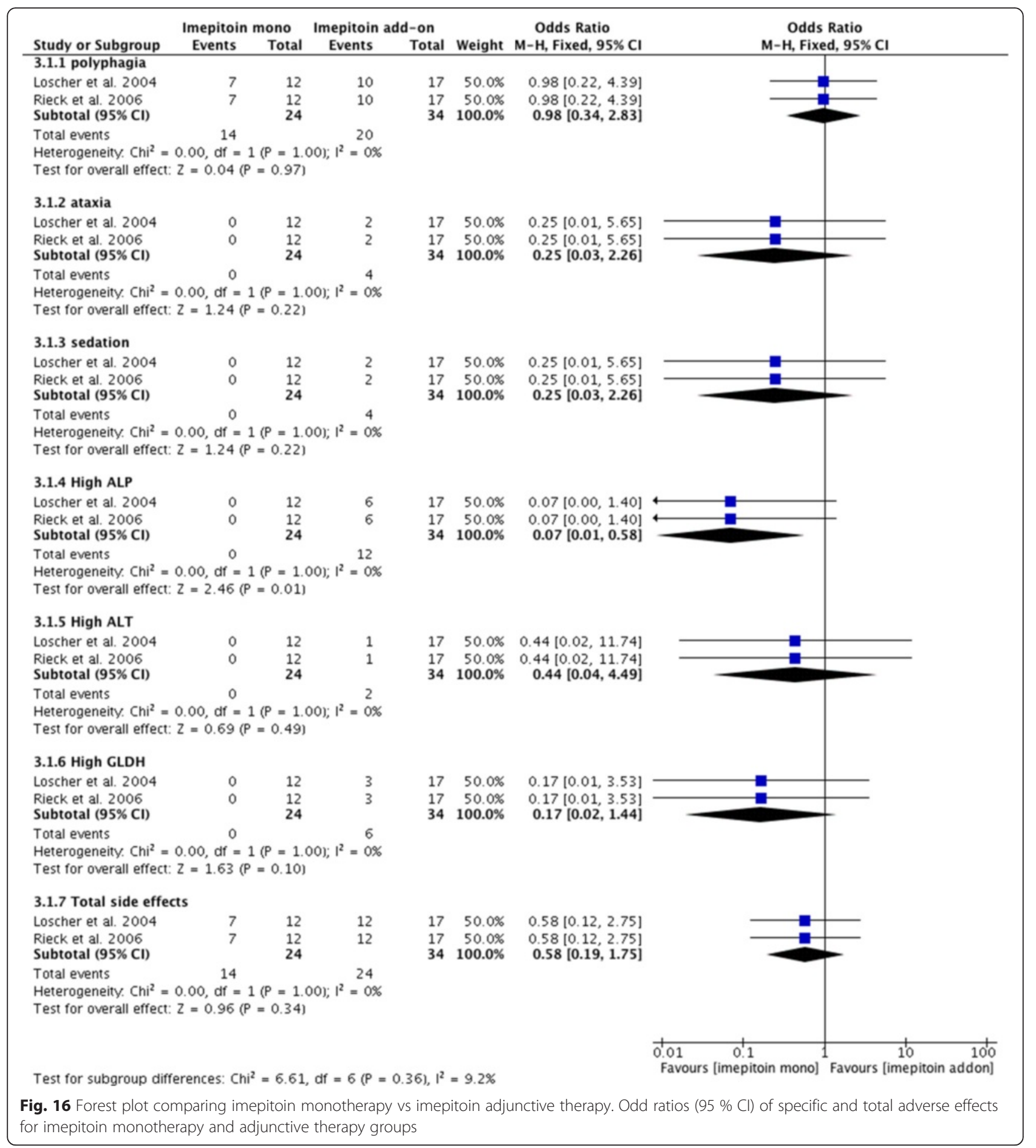

provide overall low quality evidence for many measurement outcomes (e.g. AEDs' efficacy), they can reveal valuable information as far as the safety profile is concerned and were included in the current systematic review.

\section{Difficulties in describing adverse effects among studies} It has been suggested that reporting of AEDs' adverse events in clinical trials is poor and has not improved over the years [126]. Searching for information about adverse effects can be quite complicated [127-129]. Little evidence exists on the most appropriate searching methods, and a study has assessed different methods in retrieving studies of adverse effects [4]. This study found that a combination of different search methods is required to retrieve as many studies as possible. The same study, however, has also concluded that the most 


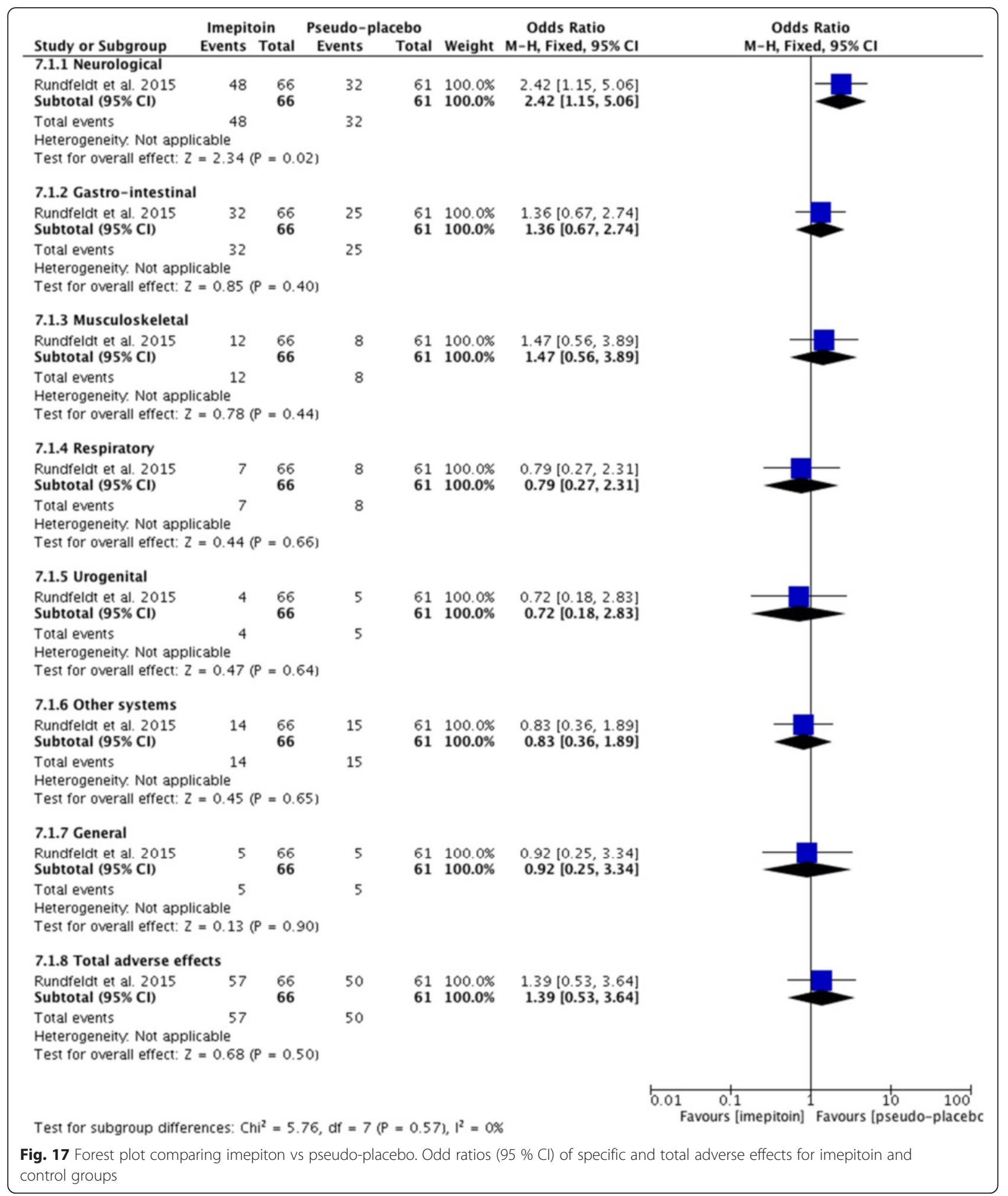

effective search methods might not be possible until there is better reporting and indexing of records concerning the adverse effects. Another recent study has indicated that database searching using adverse effects terms can retrieve the majority of articles (around $92 \%$ ) on drugs' adverse effects [130]. Our review used all the possible searching methods and combinations of these methods and terms in order to retrieve all or the vast 


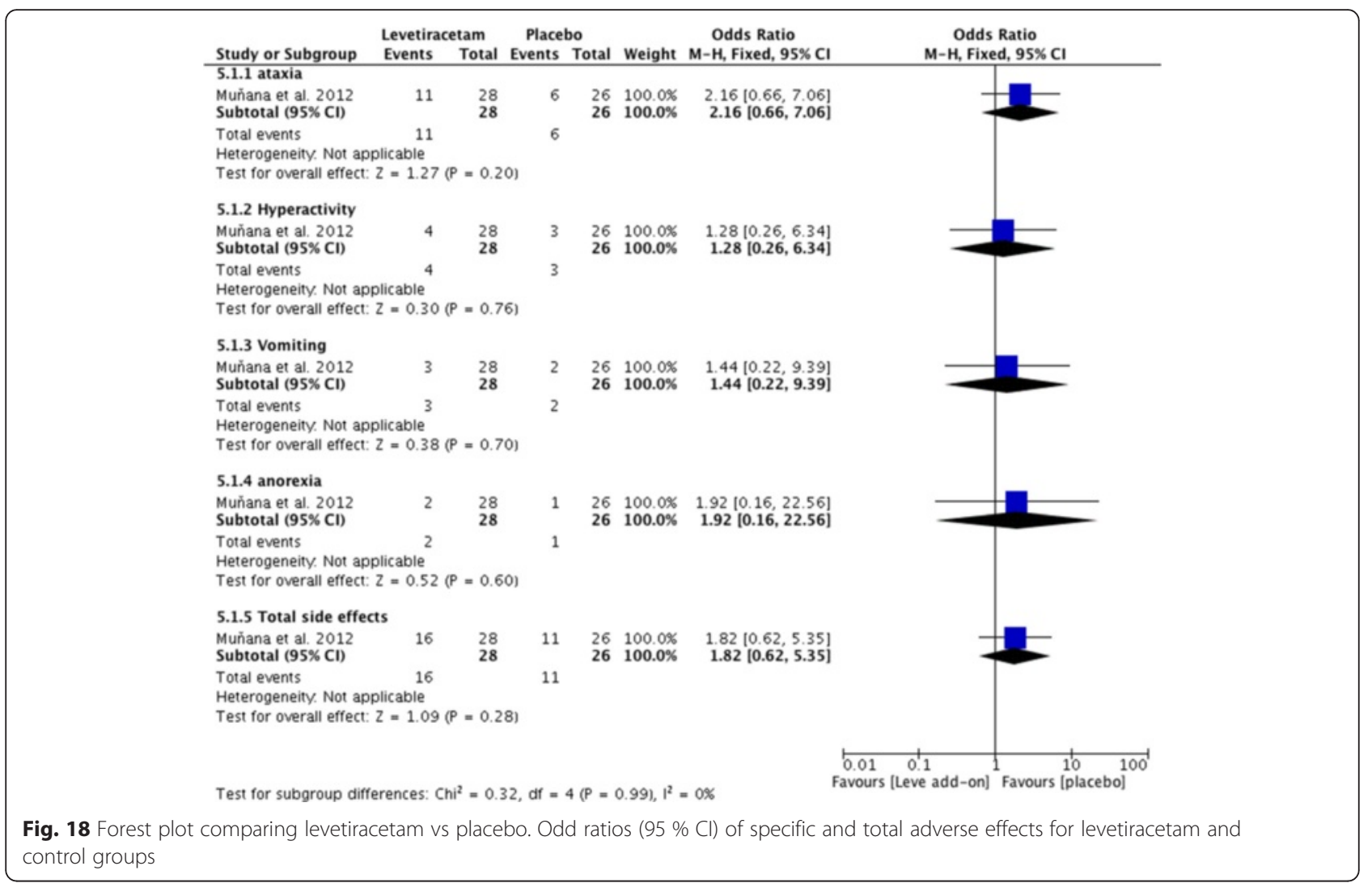

majority of the available studies reporting or assessing AEDs' adverse effects.

The majority of the studies for many AEDs, apart from phenobarbital, primidone, imepitoin and secondary potassium bromide and zonisamide, evaluated the drugs when administered in combination with other AEDs, making it sometimes difficult to determine whether clinical signs could always be attributed to AEDs' administration. Therefore, should these AEDs be used and investigated more frequently as monotherapy, future analyses (i.e. randomised controlled clinical trials) may allow even clearer conclusions on the safety questions raised in the present systematic review.

In newer standardized trials every sign that occurs during the study will be recorded as an adverse effect even if it is not always associated with the treatment. In addition, newer trials tend to include toxicology studies in which the doses that are administered are sometimes beyond the recommended treatment doses. Therefore, newer trials will report more adverse reactions than others. As imepitoin is a newly developed antiepileptic drug it is possible that the adverse effects have been more adequately captured than for older AEDs and that certain reported adverse effects might have been overreported or drug unrelated. Furthermore, a few infrequent adverse effects were recorded and mainly occurred in doses higher than the recommended therapeutic ones. The longer a drug is on the market, the likelihood of reports of adverse effects increases. This should be considered when evaluating older AEDs.

The prevalence is a good indicator for evaluating the safety profile of an AED in a study. However, a few limitations originating from the evaluated studies were detected in this review. For instance, in three studies $[31,33,76]$, the prevalence was $0 \%$, but the duration was quite short and only a very small population was included. In another study [75], although a very large population was included, the prevalence was $1 \%$, possibly because the authors focused on reporting the prevalence of pancreatitis only and the cases were reviewed retrospectively. Similarly, in 23 studies [31, 34$36,38-40,43,45,46,49-52,69,76,81,83-85,87,93$, $103]$, the authors focused on reporting only specific adverse effects and, therefore, other adverse effects may have been under- or misreported. In seven studies [36, $81,83,85,87,89,104]$, prevalence was not calculated because only dogs with adverse effects were included. In 16 studies [28, 30, 37, 39, 42, 47, 49, 54, 64, 65, 84, 85, $87,91,112,113]$, the total number of affected dogs was not very clear and therefore prevalence could not be calculated (risk of prevalence data loses). Lastly, prevalence is difficult to derive from case reports.

There have been some characteristics in the included studies that might have influenced AEDs' safety profile 
[1]. First of all, the dose range of a particular AED significantly varied among studies. Another characteristic could be the differences in AEDs' titration. The safety of some drugs can be influenced by the method and speed of titration [1]. For instance, imepitoin allows quick titration and dose adjustments in dogs with poor response to the drug. However, this was not followed in one study [25] due to the comparison with phenobarbital which demands slow titration. Therefore, this could have negatively affected imepitoin's safety profile. In addition, the number or frequency of AEDs' administration could influence safety profiles. Specifically for the AEDs with a short half-life, the number of daily administrations might affect the probability of the occurrence of adverse-effects that correlate to fluctuations of blood levels. However, it has been suggested that this characteristic is not as important as the titration speed [1].

The duration of the study should be sufficient to allow the most frequent adverse effects to occur. In many studies, the relatively small treatment period provided limited time for some adverse effects to occur. Durations of the studies were very heterogeneous and thus the spectrum of the observed adverse effects might have been affected by it. However, one human study [1], which evaluated the AEDs' central nervous system adverse effects, found that most of them occurred soon after the beginning of treatment or shortly after a dose increase. In our review, adverse effects occurred throughout the treatment duration of the studies.

As was found in the previous systematic review of AEDs' efficacy [13], several aspects may have also adversely affected the assessment of the reviewed studies. Similarly, the main aspects in the current systematic review were the difference of baseline signalment characteristics, heterogeneity in treatment initiation and protocols between studies, range of study publication dates, publication bias, the several sources of biases related to the studies, the lack of high quality evidence studies (i.e. bRCTs and bRELAS), lack of studies designed with primary aim to investigate AEDs' safety profile and enrolment of relatively small numbers of animals.

In our study, although the safety profile for each individual AED could be reported and assessed, there were factors that limit definite conclusions on safety profile among AEDs. In a few of the human systematic reviews and/or meta-analysis on AEDs' safety profile, statistical methods (e.g. comparisons of odds ratio based on study's evaluated AED and the control group, difference risk ratio, etc.) were conducted for contrasting and combining results from different groups and studies with the aim to identify similar patterns and sources of disagreement among study results or other interesting relationships that may have come to light in the context of multiple studies. In veterinary medicine, though, due to the small number of comparison (i.e. control AED or placebo) clinical trials or ELAS, lack of standardized descriptions of adverse events, variation in methods for data collecting, significant differences among study designs, several potential sources of bias and the fact that objective quantifiable measures and severity of most complaints were not considered in reports, a rigorous statistical method analysis cannot widely be used and, therefore, a meta-analytic approach is very difficult or even impossible. However, in our study, we were able to perform a statistical analysis and meta-analytic exploration for comparison groups within individual studies as well as among a few studies retrieved, which allowed us to evaluate and compare the safety profile between specific AEDs. Only a few more reliable comparisons about the safety profiles could be made, mainly, among phenobarbital, imepitoin and potassium bromide.

\section{Implications for research}

The report and assessment of the safety profile of individual AEDs on systematic reviews is very important, but it is the authors' opinion that the assessment and comparison of the safety profile among AEDs through a meta-analytic approach could offer even more valuable information and facilitate the clinician's decision on which AED to choose in respect to its safety profile. Although the prevalence of adverse effects in each study provides a general indicator of each AED's safety profile and allows limited and indirect comparisons between AEDs, the statistical analysis of comparison group studies is the essential factor that allows direct and more established comparisons. Therefore, further comparison studies are widely needed in order to perform a larger scale meta-analytic study that would offer stronger conclusions and valuable information on which AED can be considered the most or least safe. Towards this goal further conduction of high quality controlled studies (bRCTs and bRELAS) for type I adverse effects and official report of type II adverse effects are vital.

Last, but not least, a further problem that was detected by the evaluation of the studies in this review was the lack of detailed or clear information. This resulted in difficulties when performing statistics for AEDs' safety profile comparisons. Therefore, it is essential that future studies include as much accurate information as possible and that scientists have wide access to results of clinical trials and experimental studies.

\section{Implications for clinical practice}

It was found that levetiracetam might be one of the safest AEDs based on individual drug assessments, which was also supported by a strong level of evidence. Although phenobarbital is believed to be more commonly 
associated with a higher adverse effects rate compared to other AEDs such as levetiracetam or imepitoin, it could be argued that it has been longer on the market and a greater number of studies have involved phenobarbital; therefore the number of adverse event reports is likely to be higher. The current evidence based on this systematic review and the previous one [13] shows that there is good level of evidence supporting phenobarbital as one of the most effective AED, but it might have a lower safety profile than other AEDs (i.e. levetiracetam and imepitoin). There was, however, no statistically significant association between the overall safety profile of phenobarbital and imepitoin or levetiracetam. Only for sedation, hypoactivity and hyperactivity, a statistically significant difference was detected in favour of imepitoin, levetiracetam and phenobarbital respectively. Phenobarbital was not compared to further AEDs because of the lack of data (i.e. comparison studies). The remaining AEDs showed a variable safety profile that, potentially, could be high or low; no evidence neither was identified to support any of these statements, nor to compare their safety to other AEDs.

In general, most of the adverse effects reported in all AEDs, apart from the idiosyncratic ones, were not usually life threatening and subsided once doses and serum levels were reduced or following complete AED withdrawal. It is important for clinicians to be able to evaluate the benefits (i.e. the efficacy, cost and frequency of administration, pharmacokinetic properties, need for blood monitoring and lack of possible drug interactions) and risks (i.e. the potential prevalence and severity of adverse effects, long-term impact of adverse effects on patient and owner's quality of life, drug interactions) before initiating treatment with a specific AED.

\section{Conclusion}

This systematic review provides an evidence-based evaluation of the data on the AEDs' adverse effects most common usage for canine IE. Case reports were included to ensure that this review would capture idiosyncratic or other rare adverse effects. Only very few studies were designed in a randomised, controlled, and blinded manner and many of the studies included only a small study population with unclear inclusion or exclusion criteria and short term follow-up. Direct comparisons suggested that imepitoin and levetiracetam might have a better safety profile than phenobarbital, whilst the latter might have a better safety profile than potassium bromide. None of the comparisons showed a statistically significant difference. Further comparisons for all the AEDs were not possible as a considerable amount of studies did not report power calculations or clear and adequate data to allow further statistical analysis. Individual AED assessments showed that levetiracetam might be one of the safest AEDs followed by imepitoin and then phenobarbital and potassium bromide; all supported by strong level of evidence. The safety profile in other AEDs was variable but insufficient level of evidence was found to permit firm conclusions.

\section{Ethics approval and consent to participate} Not applicable.

\section{Consent for publication}

Not applicable.

\section{Availability of data and materials}

The data supporting our findings is contained within the manuscript.

\section{Abbreviations}

AED(s): anti-epileptic drug(s); bELAS: blinded experimental laboratory animal studies; BID: bis in die (twice daily); bRCTs: blinded randomised clinical trials; Chloraz: Chlorazepate; CL: confidence level; CSF: cerebrospinal fluid;

$\mathrm{CT}$ : computer tomography; CTs: clinical trials; Gaba: Gabapentin; IE: idiopathic epilepsy; Leve: Levetiracetam; m: month(s); MRI: magnetic resonance imaging; NA: Not Available; nbELAS: non-blinded experimental laboratory animal studies; nbRCTs: non-blinded randomised clinical trials; NRCTs: nonrandomised clinical trials; NRELAS: non-randomised experimental laboratory studies; OR: odds ratio; PB: phenobarbital; PBr: potassium bromide;

PD: polydipsia; PO: per os; PP: polyphagia; PU: polyuria; SF: seizure frequency; SID: semel in die (once daily); TID: ter in die (three times daily); UCTs: uncontrolled clinical trials; UELAS: uncontrolled experimental

laboratory studies; w: week(s).

\section{Competing interests}

The authors declare that they have no competing interests.

\section{Authors' contributions}

All authors helped to draft the manuscript and participated in its design. MC and SS collected the data for the study. MC assessed the data. All authors read and approved the final manuscript.

\section{Acknowledgements}

The authors would like to thank Dr Cynthia Gaskill (University of Kentucky) for assisting with our inquiries.

Funding

Not applicable.

\section{Author details}

${ }^{1}$ Faculty of Brain Sciences, UCL Institute of Neurology, University College London, London WC1E 6BT, UK. ${ }^{2}$ College of Veterinary Medicine, lowa State University, Ames, lowa 50011, USA. ${ }^{3}$ Department of Production and Population Health, Royal Veterinary College, Hawkshead Lane, Hatfield, Herts AL9 7TA, UK. ${ }^{4}$ Department of Clinical Science and Services, Royal Veterinary College, Hawkshead Lane, Hatfield, Herts AL9 7TA, UK.

Received: 18 August 2015 Accepted: 12 May 2016

Published online: 21 May 2016

\section{References}

1. Zaccara G, Gangemi PF, Cincotta M. Central nervous system adverse effects of new antiepileptic drugs. A meta-analysis of placebo-controlled studies. Seizure. 2008;17(5):405-21.

2. Baulac M: Approach of the European Medicines Agency in Development of Antiepileptic Drugs. In: Atlas of Epilepsies. edn.: Springer; 2010: 1615-1619. http://link.springer.com/referenceworkentry/10.1007/978-1-84882-128-6_243.

3. Chang Y, Mellor DJ, Anderson TJ. Idiopathic epilepsy in dogs: owners' perspectives on management with phenobarbitone and/or potassium bromide. J Small Anim Pract. 2006;47(10):574-81. 
4. Golder S, McIntosh HM, Duffy S, Glanville J, Centre for R, Dissemination, et al. Developing efficient search strategies to identify reports of adverse effects in MEDLINE and EMBASE. Health Info Libr J. 2006;23(1):3-12.

5. Loke YK. Assessing the benefit-harm balance at the bedside. BMJ. 2004; 329(7456):7-8

6. Bracchi R. Drug companies should report side effects in terms of frequency. BMJ. 1996;312(7028):442.

7. Cuervo LG, Clarke M. Balancing benefits and harms in health care. BMJ. 2003;327(7406):65-6

8. Ernst E, Pittler MH. Assessment of therapeutic safety in systematic reviews: literature review. BMJ. 2001;323(7312):546.

9. Papanikolaou PN, loannidis JP. Availability of large-scale evidence on specific harms from systematic reviews of randomized trials. Am J Med. 2004;117(8):582-9.

10. Ross SD. Drug-related adverse events: a readers' guide to assessing literature reviews and meta-analyses. Arch Intern Med. 2001;161(8):1041-6.

11. Chou R, Helfand M. Challenges in systematic reviews that assess treatment harms. Ann Intern Med. 2005;142(12 Pt 2):1090-9.

12. Berlin JA, Colditz GA. The role of meta-analysis in the regulatory process for foods, drugs, and devices. JAMA. 1999;281(9):830-4.

13. Charalambous M, Brodbelt D, Volk HA. Treatment in canine epilepsy-a systematic review. BMC Vet Res. 2014;10:257.

14. Perucca E. Clinical pharmacology and therapeutic use of the new antiepileptic drugs. Fundam Clin Pharmacol. 2001;15(6):405-17.

15. Baird-Heinz HE, Van Schoick AL, Pelsor FR, Ranivand L, Hungerford LL. A systematic review of the safety of potassium bromide in dogs. J Am Vet Med Assoc. 2012;240(6):705-15.

16. Guyatt GH, Oxman AD, Kunz R, Vist GE, Falck-Ytter Y, Schunemann HJ, Group GW. What is "quality of evidence" and why is it important to clinicians? BMJ. 2008;336(7651):995-8.

17. Straus E.S. RWS, Glasziou P., Haynes R.B.: Evidence-Based Medicine: How to Practice and Teach It, 4 edn: Churchill livingstone elsevier; 2010.

18. Holmes MA. Philosophical foundations of evidence-based medicine for veterinary clinicians. J Am Vet Med Assoc. 2009;235(9):1035-9.

19. Summers JF, Brodbelt DC, Forsythe PJ, Loeffler A, Hendricks A. The effectiveness of systemic antimicrobial treatment in canine superficial and deep pyoderma: a systematic review. Vet Dermatol. 2012;23(4):305-29. e361.

20. Higgins JPT AD, Sterne JAC et al.: In: Higgins JPT, Green S, eds. Cochrane Handbook for Systematic Reviews of Interventions Version 5.1.0. Part 2: General methods for Cochrane reviews, Chapter 8: Assessing risk of bias in included studies, Table 8.5.d: Criteria for judging risk of bias in the 'Risk of bias' assessment tool. In.: The Cochrane Collaboration; 2011. http://www. ncbi.nlm.nih.gov/pubmed/24667063.

21. Hooijmans CR, Rovers MM, de Vries RB, Leenaars M, Ritskes-Hoitinga M, Langendam MW. SYRCLE's risk of bias tool for animal studies. BMC Med Res Methodol. 2014;14:43.

22. Kirkwood B. SJ: Essential Medical Statistics, Second edn: Wiley; 2003

23. Boothe DM, Dewey C, Carpenter DM. Comparison of Phenobarbital with bromide as a first-choice antiepileptic drug for treatment of epilepsy in dogs. J Am Vet Med Assoc. 2012;240(9):1073-83.

24. Munana KR, Thomas WB, Inzana KD, Nettifee-Osborne JA, McLucas KJ, Olby NJ, Mariani CJ, Early PJ. Evaluation of levetiracetam as adjunctive treatment for refractory canine epilepsy: a randomized, placebo-controlled, crossover trial. J vet intern med / Am Coll Vet Inter Med. 2012;26(2):341-8.

25. Tipold A, Keefe TJ, Loscher W, Rundfeldt C, de Vries F: Clinical efficacy and safety of imepitoin in comparison with phenobarbital for the control of idiopathic epilepsy in dogs. J. Vet. Pharmacol. Ther. 2014;38(2):160-8.

26. Rundfeldt C, Tipold A, Loscher W. Efficacy, safety, and tolerability of imepitoin in dogs with newly diagnosed epilepsy in a randomized controlled clinical study with long-term follow up. BMC Vet Res. 2015;11:228.

27. Fredso N, Sabers A, Toft N, Moller A, Berendt M: A single-blinded phenobarbital-controlled trial of levetiracetam as mono-therapy in dogs with newly diagnosed epilepsy. Vet. J. 2015;208:44-9.

28. Schwartz-Porsche D, Loscher W, Frey HH. Therapeutic efficacy of Phenobarbital and primidone in canine epilepsy: a comparison. J Vet Pharmacol Ther. 1985;8(2):113-9.

29. Gaskill CL, Kimber WJ. Comparison of Phenobarbital and potassium bromide monotherapies in the treatment of canine epilepsy. In: ACVIM. JVIM. 2010;24:696.

30. Walker RM, DiFonzo CJ, Barsoum NJ, Smith GS, Macallum GE. Chronic toxicity of the anticonvulsant zonisamide in beagle dogs. Fundam Appl Toxicol : off j Soc Toxicol. 1988;11(2):333-42.
31. Dyer KR, Monroe WE, Forrester SD. Effects of short- and long-term administration of Phenobarbital on endogenous ACTH concentration and results of ACTH stimulation tests in dogs. J Am Vet Med Assoc. 1994;205(2): 315-8.

32. Bunch SE, Castleman WL, Baldwin BH, Hornbuckle WE, Tennant BC. Effects of long-term primidone and phenytoin administration on canine hepatic function and morphology. Am J Vet Res. 1985;46(1):105-15.

33. Daminet S, Paradis M, Refsal KR, Price C. Short-term influence of prednisone and Phenobarbital on thyroid function in euthyroid dogs. Can vet j La revue veterinaire canadienne. 1999;40(6):411-5.

34. Bunch SE, Easley JR, Cullen JM. Hematologic values and plasma and tissue folate concentrations in dogs given phenytoin on a long-term basis. Am J Vet Res. 1990;51(11):1865-8.

35. Meyer DJ, Noonan NE. Liver tests in dogs receiving anticonvulsant drugs (diphenylhydantoin and primidone). J Am Anim Hosp Assoc. 1981:17:261-4.

36. Gaskill CL, Miller LM, Mattoon JS, Hoffmann WE, Burton SA, Gelens HC, Ihle SL, Miller JB, Shaw DH, Cribb AE. Liver histopathology and liver and serum alanine aminotransferase and alkaline phosphatase activities in epileptic dogs receiving Phenobarbital. Vet Pathol. 2005; 42(2):147-60

37. Andric N, Popovic N, Stepanovic P. Francuski J, Durdevic D. Biochemical changes in the blood serum of dogs treated with Phenobarbital. Acta Vet (Beograd). 2010;60:573-84

38. Foster SF, Church DB, Watson AD. Effects of phenobarbitone on serum biochemical tests in dogs. Aust Vet J. 2000;78(1):23-6.

39. Kantrowitz LB, Peterson ME, Trepanier LA, Melian C, Nichols R. Serum total thyroxine, total triiodothyronine, free thyroxine, and thyrotropin concetrations in epileptic dogs treated with anticonvulsants. JAVMA. 1999; 214(12):1804-8

40. Gaskill CL, Burton SA, Gelens HC, Ihle SL, Miller JB, Shaw DH, Brimacombe $M B, C r i b b$ AE. Effects of Phenobarbital treatment on serum thyroxine and thyroid-stimulating hormone concentrations in epileptic dogs. J Am Vet Med Assoc. 1999;215(4):489-96.

41. Jambroszyk M, Tipold A, Potschka $\mathrm{H}$. Add-on treatment with verapamil in pharmacoresistant canine epilepsy. Epilepsia. 2011;52(2):284-91.

42. Loscher W, Hoffmann K, Twele F, Potschka H, Tollner K. The novel antiepileptic drug imepitoin compares favourably to other GABA-mimetic drugs in a seizure threshold model in mice and dogs. Pharmacol res : off j Italian Pharmacol Soc. 2013;77:39-46.

43. Litchfield $\mathrm{MH}$, Conning DM. Effect of phenobarbitone on plasma and hepatic alkaline phosphatase activity in the dog. Naunyn Schmiedebergs Arch Pharmacol. 1972;272(3):358-62.

44. Loscher W, Potschka H, Rieck S, Tipold A, Rundfeldt C. Anticonvulsant efficacy of the low-affinity partial benzodiazepine receptor agonist ELB 138 in a dog seizure model and in epileptic dogs with spontaneously recurrent seizures. Epilepsia. 2004;45(10):1228-39.

45. Muller PB, Taboada J, Hosgood G, Partington BP, VanSteenhouse JL, Taylor HW, Wolfsheimer KJ. Effects of long-term Phenobarbital treatment on the liver in dogs. J vet intern med / Am Coll Vet Inter Med. 2000;14(2):165-71

46. Muller PB, Wolfsheimer KJ, Taboada J, Hosgood G, Partington BP, Gaschen FP. Effects of long-term phenobarbital treatment on the thyroid and adrenal axis and adrenal function tests in dogs. J vet intern med / Am Coll Vet Inter Med. 2000;14(2):157-64.

47. McGee JH, Erikson DJ, Galbreath C, Willigan DA, Sofia RD. Acute, subchronic, and chronic toxicity studies with felbamate, 2-phenyl-1,3-propanediol dicarbamate. Toxicol Sci : off j Soc Toxicol. 1998;45(2):225-32.

48. Rieck S, Rundfeldt C, Tipold A. Anticonvulsant activity and tolerance of ELB138 in dogs with epilepsy: a clinical pilot study. Vet J. 2006;172(1):86-95

49. Conning DM, Litchfield MH. Increase of serum alkaline phosphatase activity due to enzyme "induction" in the liver of beagle dogs. J pathol. 1971;103(2):Pxii.

50. Steiner JM, Xenoulis PG, Anderson JA, Barr AC, Williams DA. Serum pancreatic lipase immunoreactivity concentrations in dogs treated with potassium bromide and/or phenobarbital. Vet Ther : res appl vet med. 2008;9(1):37-44

51. Paull LC, Scott-Moncrieff JC, DeNicola DB, Glickman N, Refsal KR, Glickman LT. Effect of anticonvulsant dosages of potassium bromide on thyroid function and morphology in dogs. J Am Anim Hosp Assoc. 2003;39(2):193-202.

52. Sturtevant F, Hoffmann WE, Dorner JL. The effect of three anticonvulsant drugs and ACTH on canine serum alkaline phosphatase. J Am Anim Hosp Assoc. 1977;13:754-7. 
53. Volk HA, Matiasek LA, Lujan Feliu-Pascual A, Platt SR, Chandler KE. The efficacy and tolerability of levetiracetam in pharmacoresistant epileptic dogs. Vet J. 2008;176(3):310-9.

54. Kiviranta A-MOL-V, Hielm-Björkman A, Jokinen T. Topiramate as an add-on antiepileptic drug in treating refractory canine idiopathic epilepsy. J Small Anim Pract. 2013:12130:1-9.

55. Dewey CW, Cerda-Gonzalez S, Levine JM, Badgley BL, Ducote JM, Silver GM, Cooper JJ, Packer RA, Lavely JA. Pregabalin as an adjunct to phenobarbital, potassium bromide, or a combination of phenobarbital and potassium bromide for treatment of dogs with suspected idiopathic epilepsy. J Am Vet Med Assoc. 2009;235(12):1442-9.

56. Dewey CW, Guiliano R, Boothe DM, Berg JM, Kortz GD, Joseph RJ, Budsberg SC. Zonisamide therapy for refractory idiopathic epilepsy in dogs. J Am Anim Hosp Assoc. 2004;40(4):285-91.

57. von Klopmann T, Rambeck B, Tipold A. Prospective study of zonisamide therapy for refractory idiopathic epilepsy in dogs. J Small Anim Pract. 2007; 48(3):134-8.

58. Govendir M, Perkins M, Malik R. Improving seizure control in dogs with refractory epilepsy using gabapentin as an adjunctive agent. Aust Vet J. 2005;83(10):602-8.

59. Platt SR, Adams V, Garosi LS, Abramson CJ, Penderis J, De Stefani A Matiasek L. Treatment with gabapentin of 11 dogs with refractory idiopathic epilepsy. Vet Rec. 2006;159(26):881-4.

60. Steinberg MFD. Levetiracetam therapy for long-term idiopathic epileptic dogs. J vet intern med / Am Coll Vet Inter Med. 2004;18:410.

61. Chung JY, Hwang CY, Chae JS, Ahn JO, Kim TH, Seo KW, Lee SY, Youn HY Zonisamide monotherapy for idiopathic epilepsy in dogs. N Z Vet J. 2012; 60(6):357-9.

62. Nafe LAPA, Kay WJ. Sodium valproate: a preliminary clinical trial in epileptic dogs. J Am Anim Hosp Assoc. 1981;17(1):131-3.

63. Schwartz-Porsche DJU. Wirksamkeit von Bromid bei den therapieresistenten Epilepsien des Hundes. Tierarztl Prax. 1991;19(4):395-401.

64. Cunningham JG, Haidukewych D, Jensen HA. Therapeutic serum concentrations of primidone and its metabolites, phenobarbital and phenylethylmalonamide in epileptic dogs. J Am Vet Med Assoc. 1983; 182(10):1091-4.

65. Schwartz-Porsche D, Loscher W, Frey HH. Treatment of canine epilepsy with primidone. J Am Vet Med Assoc. 1982;181(6):592-5.

66. Pearce LK. Potassium bromide as an adjunct to Phenobarbital for the management of uncontrolled seizures in dogs. Prog Vet Neurol. 1990;1:95-101.

67. Srivastava M, Singh N, Srivastava A. Management of Phenobarbital Refractory Idiopathic Epilepsy by Potassium Bromide in Dogs. Adv Anim Vet Sci. 2013;1(1):25-7

68. Boothe DM, Perkins J. Disposition and safety of zonisamide after intravenous and oral single dose and oral multiple dosing in normal hound dogs. J Vet Pharmacol Ther. 2008;31(6):544-53.

69. Aitken MM, Hall E, Scott L, Davot JL, Allen WM. Liver-related biochemical changes in the serum of dogs being treated with phenobarbitone. Vet Rec. 2003;153(1):13-6

70. Dayrell-Hart B, Tiches D, Vite C, Steinberg S: Efficacy and safety of felbamate as an anticonvulsant in dogs with refractory seizures. In: ACVIM Forum: J. Vet. Inter. Med. 1996;10:174.

71. Chauvet AE, Feldman EC, Kass PH. Effects of phenobarbital administration on results of serum biochemical analyses and adrenocortical function tests in epileptic dogs. J Am Vet Med Assoc. 1995;207(10):1305-7.

72. Moore SA, Munana KR, Papich MG, Nettifee-Osborne J. Levetiracetam pharmacokinetics in healthy dogs following oral administration of single and multiple doses. Am J Vet Res. 2010;71(3):337-41.

73. March PA, Podell M, Sams RA. Pharmacokinetics and toxicity of bromide following high-dose oral potassium bromide administration in healthy Beagles. J Vet Pharmacol Ther. 2002;25(6):425-32

74. Gaskill CL, Burton SA, Gelens HC, Ihle SL, Miller JB, Shaw DH, Brimacombe $\mathrm{MB}, \mathrm{Cribb} \mathrm{AE}$. Changes in serum thyroxine and thyroid-stimulating hormone concentrations in epileptic dogs receiving phenobarbital for one year. J Vet Pharmacol Ther. 2000;23(4):243-9.

75. Gaskill CL, Cribb AE. Pancreatitis associated with potassium bromide/ phenobarbital combination therapy in epileptic dogs. Can vet j La revue veterinaire canadienne. 2000;41(7):555-8.

76. Balazs T, Farber TM, Feuer G. Drug-induced changes in serum alkaline phosphatase and alanine aminotransferase activities not related to hepatic injuries. Archives of toxicology Supplement = Archiv fur Toxikologie Supplement. 1978;Suppl 1:159-163.

77. Podell M, Fenner WR. Bromide therapy in refractory canine idiopathic epilepsy. J vet intern med / Am Coll Vet Inter Med. 1993;7(5):318-27.

78. Ruehlmann D, Podell M, March P. Treatment of partial seizures and seizure-like activity with felbamate in six dogs. J Small Anim Pract. 2001;42(8):403-8.

79. Heynold Y, Faissler D, Steffen F, Jaggy A. Clinical, epidemiological and treatment results of idiopathic epilepsy in 54 labrador retrievers: a long-term study. J Small Anim Pract. 1997;38(1):7-14.

80. von Klopmann T, Boettcher IC, Rotermund A, Rohn K, Tipold A. Euthyroid sick syndrome in dogs with idiopathic epilepsy before treatment with anticonvulsant drugs. J vet intern med / Am Coll Vet Inter Med. 2006;20(3):516-22.

81. Bersan E, Volk HA, Ros C, De Risio L. Phenobarbitone-induced haematological abnormalities in idiopathic epileptic dogs: prevalence, risk factors, clinical presentation and outcome. Vet Rec. 2014;175(10):247.

82. Raw M, Gaskell C. A review of one hundred cases of presumed canine epilepsy. J Small Anim Pract. 1985;26:645-52.

83. March PA, Hillier A, Weisbrode SE, Mattoon JS, Johnson SE, DiBartola SP, Brofman PJ. Superficial necrolytic dermatitis in 11 dogs with a history of phenobarbital administration (1995-2002). J vet intern med / Am Coll Vet Inter Med. 2004;18(1):65-74.

84. Dayrell-Hart B, Steinberg SA, VanWinkle TJ, Farnbach GC. Hepatotoxicity of phenobarbital in dogs: 18 cases (1985-1989). J Am Vet Med Assoc. 1991; 199(8):1060-6.

85. Weiss DJ. Bone marrow necrosis in dogs: 34 cases (1996-2004). J Am Vet Med Assoc. 2005;227(2):263-7.

86. Dayrell-Hart B, Vite C, Tiches D, Aleman D, Steinberg SA: Serum bromide concentrations and seizure control in dogs with epilepsy. In: ACVIM forum: 1996: Journal of Veterinary Internal Medicine; 1996: 174.

87. Weiss DJ, Smith SA. A retrospective study of 19 cases of canine myelofibrosis. J vet intern med / Am Coll Vet Inter Med. 2002;16(2):174-8.

88. Rossmeisl JH, Inzana KD. Clinical signs, risk factors, and outcomes associated with bromide toxicosis (bromism) in dogs with idiopathic epilepsy. J Am Vet Med Assoc. 2009;234(11):1425-31.

89. Bunch SE, Castleman WL, Hornbuckle WE, Tennant BC. Hepatic cirrhosis associated with long-term anticonvulsant drug therapy in dogs. J Am Vet Med Assoc. 1982:181(4):357-62.

90. Packer RA, Nye G, Porter ES, Volk HA: Assessment into the usage of levetiracetam in a canine epilepsy clinic. BMC Vet. Res. 2015;11(25)1-8.

91. Bunch SE, Baldwin BH, Hornbuckle WE, Tennant BC. Compromised hepatic function in dogs treated with anticonvulsant drugs. J Am Vet Med Assoc. 1984;184(4):444-8.

92. Bunch SE, Conway MB, Center SA, Castleman WL, Baldwin BH, Hornbuckle WE, Tennant BC. Toxic hepatopathy and intrahepatic cholestasis associated with phenytoin administration in combination with other anticonvulsant drugs in three dogs. J Am Vet Med Assoc. 1987;190(2):194-8.

93. Bizzeti M, Croce I, Sgorbini M: Acute pancreatitis in the dog secondary to anti-convulsivant therapy with phenobarbital or with phenobarbital in association with potassium bromide. Annali Fac Med Vet. 2006;59:165-170.

94. Habock G, Pakozdy A. Hamatologische abweichungen wahrend der phenobarbital-therapie beim hund. Wien Tierarztl Monatsschr. 2012;99:242-9.

95. Farnbach GC. Serum concentrations and efficacy of phenytoin, phenobarbital, and primidone in canine epilepsy. J Am Vet Med Assoc. 1984:184(9):1117-20.

96. Stabile A, De Stefani A, De Risio L. Unusual manifestation of bromide toxicity (bromism) in an idiopathic epileptic dog already treated with phenobarbital. Vet Rec Case Rep. 2014;2:1-3.

97. Miller ML, Center SA, Randolph JF, Lepherd ML, Cautela MA, Dewey CW. Apparent acute idiosyncratic hepatic necrosis associated with zonisamide administration in a dog. J vet intern med / Am Coll Vet Inter Med. 2011; 25(5):1156-60

98. Bevier DE, Miller MA, Rohleder JJ, Wozniak AD. Pathology in practice. Superficial necrolytic dermatitis. J Am Vet Med Assoc. 2010;237(4):365-7.

99. Cook AK, Allen AK, Espinosa D, Barr J. Renal tubular acidosis associated with zonisamide therapy in a dog. J vet intern med / Am Coll Vet Inter Med. 2011:25(6):1454-7

100. Kube SA, Vernau KM, LeCouteur RA. Dyskinesia associated with oral phenobarbital administration in a dog. J vet intern med / Am Coll Vet Inter Med. 2006;20(5):1238-40. 
101. Steinmetz S, Tipold A, Bilzer T, Schenk HC. Transient neuromyopathy after bromide intoxication in a dog with idiopathic epilepsy. Irish vet j. 2012;65(1):19

102. Boynosky NA, Stokking LB. Potassium bromide-associated panniculitis. J Small Anim Pract. 2014;55(12):640-2.

103. Jacobs G, Calvert C, Kaufman A. Neutropenia and thrombocytopenia in three dogs treated with anticonvulsants. J Am Vet Med Assoc. 1998;212(5):681-4.

104. Poffenbarger EM, Hardy RM. Hepatic cirrhosis associated with long-term primidone therapy in a dog. J Am Vet Med Assoc. 1985;186(9):978-80.

105. Henricks PM. Dermatitis associated with the use of primidone in a dog. J Am Vet Med Assoc. 1987;191(2):237-8.

106. Yohn SE, Morrison WB, Sharp PE. Bromide toxicosis (bromism) in a dog treated with potassium bromide for refractory seizures. J Am Vet Med Assoc. 1992;201(3):468-70.

107. Thrift E, Wimpole JA, Malik R. Life-thratening anemia due to possible phenobarbitone idiosyncrasy in a dog. Aust Vet Practit. 2010;40(4):130-3.

108. Nash AS, Thompson H, Bogan JA. Phenytoin toxicity: a fatal case in a dog with hepatitis and jaundice. Vet Rec. 1977;100(14):280-1.

109. Mathis JC, Yoo SH, Sullivan LA. Diagnosis of secondary dysmyelopoiesis via costochondral rib aspirate in a dog. J Vet Emerg Crit Care. 2014;24(6):739-44.

110. Mackay B, Mitchell G. Spurious hyperchloraemia and negative anion gap in a dog with bromide toxicity. Aust Vet Practit. 1998;28(2):50-2.

111. von Klopmann T, Papenhagen $H$, Tipold A. Phenobarbitalinduzierte blutbildveranderungen bei einem Shi-Tzu. Tierarztl Prax. 2006;34:40-4.

112. EPAR: CVPM assesment report for pexion. European Medicines agency 2012; 1-23. http://www.ema.europa.eu/docs/en_GB/document_library/EPAR_-Public assessment report/veterinary/002543/WC500140842.pdf

113. EPAR US: CVPM assesment report for pexion. European Medicines agency. 2012;1-23. http://www.ema.europa.eu/docs/en_GB/document library/ EPAR_-_Public_assessment_report/veterinary/002543/WC500140842.pdf.

114. Speciale J, Dayrell-Hart B, Steinberg SA. Clinical evaluation of gamma-vinylgamma-aminobutyric acid for control of epilepsy in dogs. J Am Vet Med Assoc. 1991;198(6):995-1000.

115. Loke YK, Derry S, Aronson JK. A comparison of three different sources of data in assessing the frequencies of adverse reactions to amiodarone. Br J Clin Pharmacol. 2004;57(5):616-21.

116. Shaw N, Trepanier LA, Center SA, Garland S. High dietary chloride content associated with loss of therapeutic serum bromide concentrations in an epileptic dog. J Am Vet Med Assoc. 1996;208(2):234-6.

117. Tipold A: Efficacy, safety and tolerability of ELB 138 in dogs with newly diagnosed epilepsy. In EPAR. 2006

118. Heit: A clinical field study evaluating the safety and efficacy of ELB 138 in dogs with idiopathic epilepsy. In EPAR. 2011.

119. de Vries F: Efficacy of imepitoin (ELB 138) in dogs suffering from newly diagnosed idiopathic epilepsy. In EPAR. 2011.

120. Moher D, Liberati A, Tetzlaff J, Altman DG, P Group. Preferred reporting items for systematic reviews and meta-analyses: the PRISMA statement. BMJ. 2009;339:b2535.

121. Zaccara G, Franciotta D, Perucca E. Idiosyncratic adverse reactions to antiepileptic drugs. Epilepsia. 2007:48(7):1223-44

122. Navarro VJ, Senior JR. Drug-related hepatotoxicity. N Engl J Med. 2006; 354(7):731-9.

123. Papanikolaou PN, Christidi GD, loannidis JP. Comparison of evidence on harms of medical interventions in randomized and nonrandomized studies. CMAJ : Can Mil Assoc j=j de l'Assoc med can. 2006;174(5):635-41.

124. Turner RM, Spiegelhalter DJ, Smith GC, Thompson SG. Bias modelling in evidence synthesis. J R Stat Soc Ser A. 2009;172(1):21-47.

125. Aagaard L, Hansen EH. Information about ADRs explored by pharmacovigilance approaches: a qualitative review of studies on antibiotics. SSRIs and NSAIDs BMC clin pharmacol. 2009;9:4.

126. Shukralla AA, Tudur-Smith C, Powell GA, Williamson PR, Marson AG. Reporting of adverse events in randomised controlled trials of antiepileptic drugs using the CONSORT criteria for reporting harms. Epilepsy Res. 2011; 97(1-2):20-9.

127. Aronson JK, Derry S, Loke YK. Adverse drug reactions: keeping up to date. Fundam Clin Pharmacol. 2002;16(1):49-56.

128. Derry S, Loke YK, Aronson JK. Incomplete evidence: the inadequacy of databases in tracing published adverse drug reactions in clinical trials. BMC Med Res Methodol. 2001:1:7.
129. Wieland S, Dickersin K. Selective exposure reporting and Medline indexing limited the search sensitivity for observational studies of the adverse effects of oral contraceptives. J Clin Epidemiol. 2005;58(6):560-7.

130. Golder S, Loke YK. Failure or success of electronic search strategies to identify adverse effects data. J Mil Libr Assoc : JMLA. 2012;100(2):130-4.

\section{Submit your next manuscript to BioMed Central and we will help you at every step:}

- We accept pre-submission inquiries

- Our selector tool helps you to find the most relevant journal

- We provide round the clock customer support

- Convenient online submission

- Thorough peer review

- Inclusion in PubMed and all major indexing services

- Maximum visibility for your research

Submit your manuscript at www.biomedcentral.com/submit
) Biomed Central 